\title{
The Fossil Record of Elateridae (Coleoptera: Elateroidea): Described Species, Current Problems and Future Prospects
}

\author{
Robin Kundrata ${ }^{1, *,+} \mathbb{D}$, Gabriela Packova ${ }^{1,+}$, Alexander S. Prosvirov ${ }^{2} \mathbb{C}$ and Johana Hoffmannova ${ }^{1}$ \\ 1 Department of Zoology, Faculty of Science, Palacky University, 17. listopadu 50, \\ 77146 Olomouc, Czech Republic; gabriela.packova01@upol.cz (G.P.); johana.hoffmannova01@upol.cz (J.H.) \\ 2 Department of Entomology, Faculty of Biology, Moscow State University, Leninskie Gory 1/12, \\ 119234 Moscow, Russia; carrabus69@mail.ru \\ * Correspondence: robin.kundrata@upol.cz \\ + These authors contributed equally to this work.
}

Citation: Kundrata, R.; Packova, G.; Prosvirov, A.S.; Hoffmannova, J. The Fossil Record of Elateridae (Coleoptera: Elateroidea): Described Species, Current Problems and Future Prospects. Insects 2021, 12, 286. https://doi.org/10.3390/ insects 12040286

Academic Editor: Andrés Millán

Received: 1 March 2021

Accepted: 24 March 2021

Published: 25 March 2021

Publisher's Note: MDPI stays neutral with regard to jurisdictional claims in published maps and institutional affiliations.

Copyright: (c) 2021 by the authors. Licensee MDPI, Basel, Switzerland. This article is an open access article distributed under the terms and conditions of the Creative Commons Attribution (CC BY) license (https:/ / creativecommons.org/licenses/by/ $4.0 /)$.
Simple Summary: Beetle fossils play an important role in our understanding of the origin and evolutionary history of this insect order. Despite the recently increasing rate of fossil research focused on the click-beetles (Coleoptera: Elateridae), the major group in the superfamily Elateroidea, their palaeodiversity has still remained largely understudied. In this study, we summarized current knowledge on the click-beetle fossil record with a main emphasis on the described diversity. We compiled an annotated catalogue of all described fossil species in Elateridae, assessed the reliability of their systematic placement, and discuss the current state of knowledge and prospects of research of the fossil record in the group. This study should serve as a comprehensive basis for all subsequent research dealing with the origin, early evolution and diversity of Elateridae.

Abstract: The Elateridae (click-beetles) are the largest family in Elateroidea; however, their relationships, systematics and classification remain unclear. Our understanding of the origin, evolution, palaeodiversity and palaeobiogeography of Elateridae, as well as reconstruction of a reliable timecalibrated phylogeny for the group, are hampered by the lack of detailed knowledge of their fossil record. In this study, we summarize the current knowledge on all described fossil species in Elateridae, including their type material, geographic origin, age, bibliography and remarks on their systematic placement. Altogether, 261 fossil species classified in 99 genera and nine subfamilies are currently listed in this family. The Mesozoic click-beetle diversity includes 143 species, with most of them described from the Jurassic Karatau, and 118 described species are known from the Cenozoic deposits, mainly from the Eocene North American Florissant Formation and European Baltic amber. Available data on the described past diversity of Elateridae suggest that almost all fossil lineages in this group are in urgent need of revision and numerous Mesozoic species might belong to different families. Our study is intended to serve as a comprehensive basis for all subsequent research focused on the click-beetle fossil record.

Keywords: catalogue; classification; Cenozoic; click-beetles; Eucnemidae; evolution; Mesozoic; palaeodiversity; systematics

\section{Introduction}

The click-beetles (Elateridae) are the major family in Elateroidea, comprising more than 10,000 described species worldwide [1]. Despite the efforts of numerous studies using morphological or molecular data, the classification and phylogenetic relationships within the family remain far from fully understood [2-11]. Taking this into consideration, further development of click-beetle systematics and understanding their evolution would certainly benefit from integrating modern molecular-based methods and morphology with fossils into a combined phylogenetic approach. While our knowledge on the systematics and 
diversity of recent click-beetle lineages has grown considerably in recent decades, their fossil record has been only scarcely investigated, lacking any comprehensive studies [12].

The first fossil elaterid species were reported and described during the mid-19th century from European localities [13-16]. Those were usually compression fossils ranging in their age from the Upper Triassic to Miocene. Later, several other species were added from Europe and the USA [17-21]. Scudder [22-24] summarized information on fossil Coleoptera of that time, including Elateridae. Lomnicki [25] described a new species from the Miocene of Ukraine. Handlirsch [26], in his monumental work "Die fossilen Insekten und die Phylogenie der rezenten Formen", listed numerous fossil taxa in Elateridae, many of them of doubtful placement. Wickham [27,28] described the diversity of Elateridae in the Florissant deposit in Colorado, USA, and he later provided a checklist of all North American fossil beetles, including Elateridae [29]. Klebs [30] provided a checklist of elaterid genera found in Eocene North European Baltic amber. Cockerell published hundreds of papers on various taxa and fossil deposits, including several ones treating Elateridae from the Triassic of the United Kingdom [31], Burmese amber [32,33], the Eocene of the United Kingdom [34], the Paleocene of Argentina [35] or the Eocene of the USA [36]. Tillyard [37,38] and Dunstan [39] studied beetle fossils from Triassic deposits in Australia and attributed several taxa to Elateridae. Later authors continued in dealing with just a single or several species of Elateridae without any revision or a more comprehensive picture, including Ping [40] (a new monotypic genus from the Cretaceous of China), Wickham [41] (a single new species from the Eocene of the USA), Theobald [42] (a single species from the Oligocene of Germany), Piton [43] (two species from the Paleocene of France), Haupt [44] (a single species from the Eocene of Germany), Gardiner [45] (a new monotypic genus), and Becker [46] (one new genus and three new species from Miocene Mexican amber).

Iablokoff-Khnzorian [47] was the first author who provided a comprehensive revision of Elateridae in Baltic amber. In that study, he described seven genera, three subgenera and 11 species, which were also included in subsequent studies devoted to the Baltic amber [48,49]. Dolin [50] studied a Mesozoic fossil in Kyrgyzstan and described two monotypic genera which he classified in his newly established tribe Protagrypnini within Agrypninae. Later, he focused on the detailed examination of click-beetle fossils from the Jurassic deposit of Karatau, Kazakhstan. First, he elevated Protagrypnini to the subfamily level and defined three tribes in it, describing 15 species in three genera [51]. Later, he described eight species in four genera in the subfamilies Cardiophorinae and Negastriinae [52], and finally summarized the diversity of Elateridae as a whole from the Karatau deposit [53], with 107 species classified in 31 genera and five subfamilies known from that locality. Crowson [54] was the first who reported two undescribed elaterids from Cretaceous Lebanese amber. Particular attention was also paid to Chinese fossil deposits. Several click-beetles were reported from Jurassic [55,56], Cretaceous [57] and Miocene sediments [58,59]. Whalley [60] revised Jurassic insects of Dorset, England, including Elateridae, and Zaragoza-Caballero [61] descibed a new species from Mexican amber. Tröster [62-67] studied in detail Elateridae of the Eocene Grube Messel Pit and Eckfeldt Maar deposits in Germany, and focused mainly on the agrypnine genus Macropunctum Tröster, 1991. The comprehensive, illustrated catalogue "Treatise on Invertebrate Paleontology" by Carpenter [68] was an important contribution, in which he also included a list of fossil Elateridae, and placed many dubious taxa, especially those described by Handlirsch [26], as Coleoptera incertae sedis.

In the 21st century, Wappler [69] and Alekseev [70] described additional species of Eocene Macropunctum from Germany and the United Kingdom, respectively. Martins-Neto et al. [71] and Martins-Neto and Gallego [72] reported several putative elaterids from the Triassic fossil deposits in Argentina, Martin [73] described a new click-beetle species from the Jurassic of Australia, and Alekseev [74,75] and Sohn et al. [76] discovered new fossil click-beetles in the Cretaceous deposits in Russia and South Korea, respectively. In their revision of fossil Cerophytidae, Chang et al. [77] discovered that some taxa which Dolin [53] described in Elateridae are in fact cerophytids. However, the main attention regarding 
the fossil Elateridae in the 21st century was definitely paid to the Chinese Mesozoic deposits and inclusions in various ambers. Regarding the Elateridae fossils discovered in China, they belong either to Jurassic [78-81] or Cretaceous deposits [82-86] (for reviews, see [87-89]). Regarding click-beetles included in amber, their diversity in Miocene Mexican "Chiapas" amber, along with other insects, was summarized by Solórzano Kraemer [90]. Schimmel [91] described several new species of Megapenthini from North European Baltic amber. Alekseev [92] provided a checklist of beetles described from that amber, and later Kirejtshuk and Kovalev [93] added the first representative of Omalisidae (currently the elaterid subfamily Omalisinae [7]), and Kundrata et al. [94] described a new genus in the lissomine tribe Protelaterini. Kirejtshuk and Azar [95] reported several unnamed Elateridae from Cretaceous Lebanese amber. Otto [96] added a new genus from Cretaceous Burmese amber, which he classified in Pityobiinae.

A comprehensive online checklist of fossil beetles by Kirejtshuk and Ponomarenko [97] also covers Elateridae but requires some updates, including changes in classification. Oberprieler et al. [98] reported a possible elaterid fossil from the Upper Jurassic of Australia. Most recently, Kundrata et al. [12] compiled an updated comprehensive summary of the fossil genera in Elateridae, including their systematic placement and information on the type species, gender, number of species, age range, and relevant bibliography, and Muona et al. [99] revised the clicking Elateroidea from Chinese Mesozoic deposits, with several former click-beetle taxa transferred to Throscidae and Eucnemidae. This study is a follow up to the annotated catalogue of fossil genera in Elateridae [12]. Our understanding of the origin, evolution, palaeodiversity and palaeobiogeography of Elateridae, as well as the reconstruction of a reliable time-calibrated phylogeny for the group, are hampered by the lack of a detailed investigation of their known fossil record. Therefore, this study aims to summarize the current knowledge on all described fossil species in Elateridae, including their synonyms, misspellings, type material, geographic origin, age, bibliography and their systematic placement according to the most recent publications. It should serve as a comprehensive basis for all subsequent research dealing with a click-beetle fossil record, including the studies on the early evolution and diversity of Elateridae.

\section{Materials and Methods}

We compiled information on all fossil species in Elateridae. Inclusions in the Holocene copal sensu Solórzano Kraemer et al. [100] were not included in this study as they most probably represent recent species [101-105]. The higher classification of Elateridae follows Kundrata et al. [6,12]. The compositions of the subfamilies and tribes follow Johnson [106], Cate [107], Douglas [108], Kundrata et al. [12,94,109], and citations therein. We follow the style used in the first part of the World catalogue of the genus-group names in extant Elateridae [109] and the catalogue of the genus-group names in fossil Elateridae [12]. The names of the family, genus- and species-group taxa are given with the name of the author, and the year and page of publication. The page given is the page where the taxon name and description are printed. The year and page given for the incorrect subsequent spellings are the first year and page in which they are used. The detailed information for family group names is given in Bouchard et al. [110] and that for genus-group names (including synonyms, misspellings, unavailable names, type species and their designations) can be found in various publications and catalogues $[12,107,109,111,112]$ and is not repeated here. Only generic misspellings and unavailable names which were not included in the catalogue of fossil genera [12] are reported here, and these are given under genusnames only and are not repeated under species-names. Misspellings and unavailable names are followed by colon ":". For each fossil species in Elateridae, we provide all synonyms, information on the type series and type depositories, fossil deposit and age, and relevant bibliography. Information on the type depositories was taken from the original descriptions, the museum webpages, curators, or from the Paleobiology Database (https:// paleobiodb.org/; accessed on 10 October 2020). Taxa marked with an asterisk $\left(^{*}\right)$ also contain recent species. The age of fossils was taken from the Paleobiology Database 
(https:/ / paleobiodb.org/; accessed on 10 October 2020). Divisions of geological time and their boundaries follow the ICS International Chronostratigraphic Chart v. 2020/03 (http://www.stratigraphy.org/; accessed on 10 October 2020) [113]. We assessed each fossil species based on its original description and available illustrations to conclude whether its position in Elateridae and its generic attribution can be considered reliable or not. Most doubtful family or generic placements are discussed within the Remark section under the particular species. General information about species within the same genus are given in the Remark section under the particular genus. Proper taxonomic placement of listed fossil click-beetle lineages needs a detailed investigation of the type material which was far beyond the scope of this study, and hence the remarks on potential systematic misplacement of some taxa do not represent formal taxonomic decisions unless stated otherwise. An overview of the fossil Elateridae is summarized in Appendix A (Table A1).

\section{Abbreviations}

Abbreviations for museums and collections:

BMNH Natural History Museum, London, The United Kingdom

CNU Key Laboratory of Insect Evolution and Environmental Changes, College of Life Science,

$\mathrm{CNU}$ Capital Normal University, Beijing, China

CUB University of Colorado Museum of Natural History, Boulder, Colorado, USA

ETH Swiss Federal Institute of Technology, Zurich, Switzerland

FIS Forschungsstation Grube Messel of the Senckenberg Forschungsinstitut und

FIS Naturmuseum, Frankfurt am Main, Germany (= SMF)

GIH Geologisches Institut Halle (Saale), Germany

GNUE Gongju National University of Education, Gongju, South Korea

GPIBO Steinmann Institute for Geology, Mineralogy and Palaeontology, University of Bonn,

Germany

GPIUH Geological-Paleontological Museum of the University of Hamburg, Germany

GSC Geological Survey of Canada, Ottawa, Canada

HLMD Hessisches Landesmuseum, Darmstadt, Germany

MCZ Museum of Comparative Zoology, Harvard University, Cambridge, Massachusetts, USA

MNHN Museum National d'Histoire Naturelle, Paris, France

NHMB Naturhistorisches Museum, Basel, Switzerland

NHMM Naturhistorisches Museum Mainz, Mainz, Germany

NIGP Nanjing Institute of Geology and Palaeontology, Nanjing, China

PIN Palaeontological Institute of the Russian Academy of Sciences, Moscow, Russia

QM Queensland Museum, Brisbane, Australia

SGMS Shandong Geological Museum, Jinan, Shandong, China

SMJS Shandong Museum (= Shandong Provincial Museum), Jinan, Shandong, China

SMNH Swedish Museum of Natural History (Naturhistoriska Riksmuseet), Stockholm, Sweden

SMNK Staatliches Museum für Naturkunde Karlsruhe, Germany

SNAA Stiftung Naturama Aargau, Aarau, Switzerland

UCMP University of California Museum of Paleontology, Berkeley, California, USA

UNAM Instituto de Biología, Universidad Nacional Autónoma de México, Mexico

USNM Smithsonian Institution, National Museum of Natural History, Washington, D.C., USA

UZH University of Zurich, Switzerland

WAM Invertebrate Paleontology collection, Western Australian Museum, Australia

WIRC Wisconsin Insect Research Collection, Department of Entomology at the University of

WIRC Wisconsin, Madison, Wisconsin, USA

YPM Yale Peabody Museum of Natural History, New Haven, Connecticut, USA

ZIN Zoological Institute of Russian Academy of Sciences, St. Petersburg, Russia

\section{Results}

Family Elateridae Leach, 1815 *

Elaterides Leach, 1815: 85 [114]. Type genus: Elater Linnaeus, 1758: 404 [115]. For more information, including synonyms, see Bouchard et al. [110] and Kundrata et al. [109]. 


\subsection{Subfamily Agrypninae Candèze, 1857 *}

Agrypnides Candèze, 1857: 17 [116]. Type genus: Agrypnus Eschscholtz, 1829: 32 [117]. For more information, including synonyms, see Kundrata et al. [109].

\subsubsection{Tribe Agrypnini Candèze, 1857 *}

Agrypnides Candèze, 1857: 17 [116]. Type genus: Agrypnus Eschscholtz, 1829: 32 [117]. For more information, including synonyms, see Kundrata et al. [109].

\section{- Genus Adelocera Latreille, 1829*}

Adelocera Latreille, 1829: 451 [118]. Type species: Elater ovalis Germar, 1823: 49 [119] (ICZN application needed). For more information, including synonyms, see Kundrata et al. [109].

\section{Adelocera perantiqua Cockerell and LeVeque, 1931}

Adelocera perantiqua Cockerell and LeVeque, 1931: 359 [36].

Type material. Holotype, sex unknown, compression fossil, No. 15,571 (CUB).

Fossil deposit/age. USA: Colorado, Green River Formation, Station 20, Roan Plateau; 50.3-46.2 Ma (Eocene).

Literature. Cockerell and LeVeque (1931: 359): original description [36].

Remark. According to the body proportions, shape of antenna and structure of thorax (e.g., pronotosternal sutures apparently closed, posterior angles of pronotum sharp and long, and prosternal process long, acute and with a tooth), this species does not belong neither to the currently defined genus Adelocera nor to the tribe Agrypnini. Based on the general habitus, long posterior angles of pronotum and marked elytra, it might be a member of Agrypninae: Oophorini. We prefer to postpone any taxonomic changes pending a comprehensive review including the type material.

\section{- $\quad$ Genus Ageratus Dolin, 1980}

Ageratus Dolin, 1980: 72 [53]. Type species: Ageratus ponomarenkoi Dolin, 1980: 73 [53]. For more information, see Kundrata et al. [12].

Remark. Type species of Ageratus is morphologically similar to the genera currently classified in Pseudomelanactini, and, therefore, the systematic position of this genus needs further examination.

\section{Ageratus delicatus Dolin, 1980}

Ageratus delicatus Dolin, 1980: 73 [53]. (PIN).

Type material. Holotype, sex unknown, exoskeleton, compression fossil, No. 2452/87

Fossil deposit/age. Kazakhstan: Karabastau Formation, Karatau, Galkino; 166.1-157.3 Ma (Jurassic).

Literature. Dolin (1980: 73): original description [53]; Korneev and Cate (2005: 14): checklist [120].

Remark. This species differs from the type species of Ageratus in the body proportions and smaller body size, relatively shorter and broader prothorax, and the presence of long sublateral carinae on pronotum. Based on these characters, A. delicatus reminds representatives of genera Agrypnus Eschscholtz, 1829 or Compsolacon Reitter, 1905.

Ageratus ponomarenkoi Dolin, 1980

Ageratus ponomarenkoi Dolin, 1980: 73 [53].

Type material. Holotype, sex unknown, exoskeleton, compression fossil, No. 1739/35 (part + counterpart) (PIN).

Fossil deposit/age. Kazakhstan: Karabastau Formation, Karatau, Galkino; 166.1-157.3 Ma (Jurassic). 
Literature. Dolin (1980: 73): original description [53]; Carpenter (1992: 304): generic catalogue [68]; Korneev and Cate (2005: 9): checklist [120]; Kundrata et al. (2020: 5): generic catalogue [12].

Remark. This species is similar in the shape of antennae, the elongated prothorax with deep, fully excavated pronotosternal sutures, and the elongated elytra to genera Lanelater Arnett, 1952 and Anthracalaus Fairmaire, 1888 (Agrypninae: Pseudomelanactini). Its placement in Agrypnini should be re-evaluated.

\section{- Genus Agrypnus Eschscholtz, 1829*}

Agrypnus Eschscholtz, 1829: 32 [117]. Type species: Elater murinus Linnaeus, 1758: 406 [115]. For more information, including synonyms, see Sánchez-Ruiz [121] and Kundrata et al. [109].

Agrypnus exhumatus (Wickham, 1916), comb. nov.

Lacon exhumatus Wickham, 1916: 501 [28].

Type material. Holotype, sex unknown, exoskeleton, compression fossil, No. 2776, No. 4456 in Scudder coll. (MCZ).

Fossil deposit/age. USA: Colorado, Florissant Formation, Florissant; 37.2-33.9 Ma (Eocene).

Literature. Wickham (1916: 501): original description [28]; Wickham (1920: 354): catalogue [29].

Remark. This species was originally compared with Lacon rectangularis (Say, 1825) [28], which has been currently classified in Agrypnus. Lacon exhumatus Wickham, 1916 morphologically fits to the genus Agrypnus sensu Hayek [122].

\section{- Genus Compsoderus Dolin, 1980}

Compsoderus Dolin, 1980: 71 [53]. Type species: Compsoderus priscus Dolin, 1980: 72 [53]. For more information, see Kundrata et al. [12].

Remark. This genus might represent Eucnemidae based on the habitus and shape of prothorax; however, the type specimen should be examined to find supporting characters as defined by Muona et al. [99].

\section{Compsoderus priscus Dolin, 1980}

Compsoderus priscus Dolin, 1980: 72 [53]. (PIN).

Type material. Holotype, sex unknown, exoskeleton, compression fossil, No. 2066/2975

Fossil deposit/age. Kazakhstan: Karabastau Formation, Karatau, Mikhailovka; 166.1157.3 Ma (Jurassic).

Literature. Dolin (1980: 72): original description [53]; Carpenter (1992: 304): generic catalogue [68]; Korneev and Cate (2005: 10): checklist [120]; Kundrata et al. (2020: 5): generic catalogue [12].

\section{- Genus Lacon Laporte, 1838 *}

Lacon Laporte, 1838: 11 [123]. Type species: Elater atomarius Fabricius, 1798: 139 [124] (=Elater punctatus Herbst, 1784: 110 [125]). For more information, including synonyms, see Kundrata et al. [109].

Lacon granulatus (Heer, 1847), comb. nov.

Adelocera granulata Heer, 1847: 139 [14].

Type material. Holotype, sex unknown, compression fossil (SNAA).

Fossil deposit/age. Germany: Upper Freshwater-Molasse Formation, Öhningen; 12.7-11.608 Ma (Miocene). 
Literature. Heer (1847: 139): original description [14]; Giebel (1852: 651): catalogue [126]; Giebel (1856: 96): redescription [16]; Scudder (1891: 459): catalogue [24]; Handlirsch (1907: 743): catalogue [127]; Piton (1940: 179): remark [43].

Remark. Based on the generic diagnoses of Adelocera and Lacon by Hayek [122] (see also $[111,128]$ ), we transfer $A$. granulata to Lacon. However, this placement should be taken as tentative due to the absence of crucial diagnostic characters in the fossil.

Lacon jungi (Piton, 1940), comb. nov.

Adelocera jungi Piton, 1940: 178 [43].

Type material. Holotype, sex unknown, exoskeleton, compression fossil, No. 76 (MNHN).

Fossil deposit/age. France: Menat Formation, Menat; 61.6-59.2 Ma (Paleocene).

Literature. Piton (1940: 178): original description [43].

Remark. Based on the general habitus, the length and shape of antenna, and the shape of pronotum, we transfer A. jungi to Lacon. For the generic diagnoses of Adelocera and Lacon see Hayek [122].

\section{Lacon primordialis Heer, 1847}

Lacon primordialis Heer, 1847: 138 [14].

Type material. Holotype, sex unknown, compression fossil, No. 7887 (ETH).

Fossil deposit/age. Germany: Upper Freshwater-Molasse Formation, Öhningen; 12.7-11.608 Ma (Miocene).

Literature. Heer (1847: 138): original description [14]; Giebel (1852: 651): catalogue [126]; Giebel (1856: 96): redescription [16]; Scudder (1891: 544): catalogue [24]; Handlirsch (1907: 743): catalogue [127].

Remark. Based on the body proportions and the closed pronotosternal sutures, this species does not fit into the current diagnosis of neither the genus Lacon nor the tribe Agrypnini [122]. Superficially this species resembles Dendrometrinae sensu lato [6]. However, we prefer to postpone any taxonomic changes pending a comprehensive review including the type material.

\section{- Genus Litholacon Dolin, 1980}

Litholacon Dolin, 1980: 67 [53]. Type species: Litholacon derumpens Dolin, 1980: 68 [53]. For more information, see Kundrata et al. [12].

Remark. This genus is in a need of revision since some species strongly differ from the type species (and also from each other) in various diagnostic characters, e.g., the body proportions and shapes of antenna, prothorax and elytra. Several species, especially those with closed pronotosternal sutures, which is a character typical for Agrypnini, resemble representatives of Negastriinae or Dendrometrinae: Hypnoidini. However, many species of this genus, if not all, might be in fact Eucnemidae, which is suggested by their rather short and broad thorax (in some species with furrowed pronotosternal sutures), short and broad prosternal process, compact elytra, and the shape of antenna.

\section{Litholacon conicicollis Dolin, 1980}

Litholacon conicicollis Dolin, 1980: 70 [53].

Type material. Holotype, sex unknown, exoskeleton, compression fossil, No. 2784/1371 (PIN).

Fossil deposit/age. Kazakhstan: Karabastau Formation, Karatau, Mikhailovka; 166.1157.3 Ma (Jurassic).

Literature. Dolin (1980: 70): original description [53]; Korneev and Cate (2005: 14): checklist [120].

Remark. Pronotosternal sutures of this species are closed (Figure 76 in [53]) which does not correspond neither with the diagnosis of genus Litholacon nor with Agrypnini. 


\section{Litholacon derumpens Dolin, 1980}

Litholacon derumpens Dolin, 1980: 67/68 [53]. (PIN).

Type material. Holotype, sex unknown, exoskeleton, compression fossil, No. 2554/699

Fossil deposit/age. Kazakhstan: Karabastau Formation, Karatau, Mikhailovka; 166.1157.3 Ma (Jurassic).

Literature. Dolin (1980: 68): original description [53]; Carpenter (1992: 305): generic catalogue [68]; Korneev and Cate (2005: 10): checklist [120]; Kundrata et al. (2020: 5): generic catalogue [12].

Remark. This species was designated as the type species of Litholacon ([53], p. 67) and the concept of the species is clear from Figure 72 (without a page number), with the legend as follows: "Litholacon derumpens Dolin, sp. nov., holotype Nr. 2554/699". Dolin obviously described L. derumpens on page 68 (based on the holotype number and reference to Figure 72) but under the name L. panphilovi, which he used also for the other species on the same page.

\section{Litholacon exilis Dolin, 1980}

Litholacon exilis Dolin, 1980: 71 [53]. (PIN).

Type material. Holotype, sex unknown, exoskeleton, compression fossil, No. 2997/2005

Fossil deposit/age. Kazakhstan: Karabastau Formation, Karatau, Mikhailovka; 166.1157.3 Ma (Jurassic).

Literature. Dolin (1980: 71): original description [53]; Korneev and Cate (2005: 15): checklist [120].

Remark. Pronotosternal sutures of this species are closed (Figure 78 in [53]) which does not correspond neither with the diagnosis of genus Litholacon nor with Agrypnini. The systematic placement of this species requires further study.

\section{Litholacon major Dolin, 1980}

Litholacon major Dolin, 1980: 71 [53].

Type material. Holotype, sex unknown, exoskeleton, compression fossil, No. 2239/1452 (part + counterpart) (PIN).

Fossil deposit/age. Kazakhstan: Karabastau Formation, Karatau, Mikhailovka; 166.1157.3 Ma (Jurassic).

Literature. Dolin (1980: 71): original description [53]; Korneev and Cate (2005: 19): checklist [120].

\section{Litholacon panphilovi Dolin, 1980}

Litholacon panphilovi Dolin, 1980: 68 [53].

Type material. Holotype, sex unknown, exoskeleton, compression fossil, No. 2066/2400 (PIN). Paratype, sex unknown, exoskeleton, compression fossil, No. 2239/1407.

Fossil deposit/age. Kazakhstan: Karabastau Formation, Karatau, Mikhailovka; 166.1157.3 Ma (Jurassic).

Literature. Dolin (1980: 68): original description [53]; Korneev and Cate (2005: 21): checklist [120].

\section{Litholacon ohiri Dolin, 1980}

Litholacon ohiri Dolin, 1980: 69 [53].

Type material. Holotype, sex unknown, exoskeleton, compression fossil, No. 2997/420 (PIN).

Fossil deposit/age. Kazakhstan: Karabastau Formation, Karatau, Mikhailovka; 166.1157.3 Ma (Jurassic).

Literature. Dolin (1980: 69): original description [53]; Korneev and Cate (2005: 21): checklist [120]. 
Remark. Pronotosternal sutures of this species are closed (Figure 74 in [53]) which does not correspond neither with the diagnosis of genus Litholacon nor with Agrypnini. The systematic placement of this species requires further study.

\section{Litholacon petrorsus Dolin, 1980}

Litholacon petrorsus Dolin, 1980: 69 [53]. (PIN).

Type material. Holotype, sex unknown, exoskeleton, compression fossil, No. 2066/2456

Fossil deposit/age. Kazakhstan: Karabastau Formation, Karatau, Mikhailovka; 166.1157.3 Ma (Jurassic).

Literature. Dolin (1980: 69): original description [53]; Korneev and Cate (2005: 21): checklist [120].

\section{- Genus Macropunctum Tröster, 1991}

Macropunctum Tröster, 1991: 100 [62]. Type species: Macropunctum messelense Tröster, 1991: 106 [62]. For more information, see Kundrata et al. [12].

Remark. This genus is similar in the body proportions and the structure of thorax and elytra to the Nearctic Agrypnus rectangularis (Say, 1825) and related species (previously assigned under a separate genus Colaulon Arnett, 1952). The relationships between these groups require more detailed examination.

\section{Macropunctum angulosum Tröster, 1999}

Macropunctum angulosum Tröster 1999: 13 [67].

Type material. Holotype, sex unknown, exoskeleton, compression fossil, SMF MeI 4120 (FIS). Two paratypes, exoskeletons, compression fossils, sex unknown, SMF MeI 381, 796 (FIS).

Fossil deposit/age. Germany: Messel Formation, Grube Messel Pit (type locality: E10, 3.52-3.66 below alpha); 48.6-40.4 Ma (Eocene).

Literature. Tröster (1999: 13): original description [67]; Wappler (2003: 88): revision [69].

\section{Macropunctum angustiscutellum Tröster, 1994}

Macropunctum angustiscutellum Tröster, 1994: 154 [66].

Type material. Holotype, sex unknown, exoskeleton, compression fossil, SMF MeI 2571 (FIS). 10 paratypes, sex unknown, exoskeletons, compression fossils, SMF MeI 637, $3357,233,253,373,794,1109,2262,163$ (originally as a paratype of M. messelense), 271 (originally as a paratype of M. messelense) (FIS).

Fossil deposit/age. Germany: Messel Formation, Grube Messel Pit (type locality: E9, 0.43-0.97 $\mathrm{m}$ above alpha); 48.6-40.4 Ma (Eocene).

Literature. Tröster (1994: 154): original description [66]; Wappler (2003: 89): revision [69].

\section{Macropunctum densipunctum Wappler, 2003}

Macropunctum densipunctum Wappler, 2003: 87 [69].

Macropunctum densepunctum: Kirejtshuk et al., 2019: 48 [70] [unavailable name, incorrect subsequent spelling not in prevailing usage; [129], Art. 33.3].

Type material. Holotype, sex unknown, exoskeleton, PE_2000/955, LS (NHMM). Paratype, sex unknown, exoskeleton, abdomen, PE_2000/744 a+b, LS (NHMM).

Fossil deposit/age. Germany: Eifel Formation, Eckfeld Maar; 48.6-40.4 Ma (Eocene).

Literature. Wappler (2003: 87): original description [69]; Kirejtshuk et al. (2019: 48): remark [70]. 
Type material. Holotype, sex unknown, exoskeleton, compression fossil, PE 1990/974LS (NHMM).

Fossil deposit/age. Germany: Eifel Formation, Eckfeld Maar; 48.6-40.4 Ma (Eocene).

Literature. Tröster (1992: 114): original description [63]; Wappler (2003: 89): revision [69].

Macropunctum eocaenicum (Meunier, 1921)

Ancylochira eocaenica Meunier, 1921: 7 [130].

Macropunctum eocaenicum: Tröster, 1991: 102 [62].

Type material. Lectotype, sex unknown, exoskeleton, compression fossil, HLMD Me 1082 (HLMD). Nine paralectotypes, sex unknown, exoskeletons, compression fossils, HLMD 937, 1225, 1338, 593, 1182, 4090, 1393, 925, 927 (HLMD).

Fossil deposit/age. Germany: Messel Formation, Grube Messel Pit; 48.6-40.4 Ma (Eocene).

Literature. Meunier (1921: 7): original description [130]; Tröster (1991: 102): revision [62]; Tröster (1994: 148): remark [66]; Wappler (2003: 89): revision [69]; Schimmel (2005: 27): remark [91]; Schimmel and Tarnawski (2010: 363): remark [131]; Schimmel and Tarnawski (2012: 265): remark [132].

\section{Macropunctum latiscutellum Tröster, 1994}

Macropunctum latiscutellum Tröster, 1994: 151 [66].

Type material. Holotype, sex unknown, exoskeleton, compression fossil, SMF MeI 628 (originally as a paratype of M. messelense) (FIS). 13 paratypes, sex unknown, exoskeletons, compression fossils, SMF MeI 696 (originally as a paratype of M. messelense), 2671, 5, 14, 230, 244, 256, 627, 1055, 1399, 2591, 2882, 3411 (FIS).

Fossil deposit/age. Germany: Messel Formation, Grube Messel Pit (type locality: F11, $1.0 \mathrm{~m}$ above alpha); 48.6-40.4 Ma (Eocene).

Literature. Tröster (1994: 151): original description [66].

\section{Macropunctum messelense Tröster, 1991}

Macropunctum messelense Tröster, 1991: 106 [62].

Type material. Holotype, sex unknown, exoskeleton, compression fossil, SMF MeI 2392 (FIS). 10 paratypes, sex unknown, exoskeletons, compression fossils, SMF MeI 675, 2293, 8, 760, 911, 1103, 1132, 1351, 2487, 2490 (FIS).

Fossil deposit/age. Germany: Messel Formation, Grube Messel Pit (type locality: E11, 5.06-5.71 m below alpha); 48.6-40.4 Ma (Eocene).

Literature. Tröster (1991: 106): original description [62]; Tröster (1994: 148): remark [66]; Tröster (1999: 13): remark [67]; Wappler (2003: 89): revision [69]; Kirejtshuk et al. (2019: 48): remark [70]; Kundrata et al. (2020: 5): generic catalogue [12].

\section{Macropunctum meunieri Tröster, 1991}

Macropunctum meunieri Tröster, 1991: 112 [62].

Type material. Holotype, sex unknown, exoskeleton, compression fossil, SMF MeI 2627 (FIS). Four paratypes, sex unknown, exoskeletons, compression fossils, SMF MeI 430, 536, 669, 2136 (FIS).

Fossil deposit/age. Germany: Messel Formation, Grube Messel Pit (type locality: grid square i8, 0.26-1.29 $\mathrm{m}$ above alpha); 48.6-40.4 Ma (Eocene).

Literature. Tröster (1991: 112): original description [62]; Wappler (2003: 89): revision [69].

Macropunctum minutum (Meunier, 1921)

Ancylochira minuta Meunier, 1921: 8 [130].

Macropunctum minutum: Tröster, 1994: 160 [66]. 
Type material. Lectotype, sex unknown, exoskeleton, compression fossil, HLMD Me 1206. (HLMD). Two paralectotypes reported by Tröster [66], sex unknown, exoskeletons, compression fossils, HLMD Me 1276 and 1124 (HLMD). Additional 12 specimens listed in the original description $(988,877,1087,1261,1227,1263,1229,1140,1077,1226,4142$, 648) [130].

Fossil deposit/age. Germany: Messel Formation, Grube Messel Pit; 48.6-40.4 Ma (Eocene).

Literature. Meunier (1921: 8): original description [130]; Tröster (1994: 160): remark, nomenclature [66].

\section{Macropunctum promptum (Meunier, 1921)}

Ancylochira prompta Meunier, 1921: 8 [130].

Macropunctum promptum: Tröster, 1991: 105 [62].

Type material. Holotype, sex unknown, exoskeleton, compression fossil, HMLD Me 1003 (HLMD). Eight paratypes, sex unknown, exoskeletons, compression fossils, HMLD Me 755, 1330, 650, 1093, 809, 932, 1184, 952 (HLMD). Both Meunier [130] and Tröster [62] listed nine paratypes but No. 650 was mentioned twice.

Fossil deposit/age. Germany: Messel Formation, Grube Messel Pit; 48.6-40.4 Ma (Eocene).

Literature. Meunier (1921: 8): original description [130]; Tröster (1991: 105): remark [62], Tröster (1994: 160): remark [66].

\section{Macropunctum rebugense Tröster, 1994}

Macropunctum rebugense Tröster, 1994: 158 [66].

Type material. Holotype, sex unknown, exoskeleton, compression fossil, SMF MeI 1145 (FIS). Three paratypes, sex unknown, exoskeletons, compression fossils, SMF MeI 143, 699, and 3474 (FIS).

Fossil deposit/age. Germany: Messel Formation, Grube Messel Pit (type locality: 5, E15, $1 \mathrm{~m}$ below to $2 \mathrm{~m}$ above alpha); 48.6-40.4 Ma (Eocene).

Literature. Tröster (1994: 158): original description [66]; Wappler (2003: 89): revision [69].

\section{Macropunctum rossi Alekseev, 2019}

Macropunctum rossi Alekseev in Kirejtshuk et al., 2019: 48 [70].

Type material. Holotype, sex unknown, exoskeleton, compression fossil, NHMUK I.10085 (BMNH).

Fossil deposit. United Kingdom: England, Isle of Wight, Bouldnor Formation, Bembridge Marls; 38.0-33.9 Ma (Eocene).

Literature. Kirejtshuk et al. (2019: 48): original description [70].

\section{Macropunctum senckenbergi Tröster, 1994}

Macropunctum senckenbergi Tröster, 1994: 148 [66].

Type material. Holotype, sex unknown, exoskeleton, compression fossil, SMF MeI 297 (FIS). Four paratypes, sex unknown, exoskeletons, compression fossils, SMF MeI 672, 2549, 3225, 3232 (FIS).

Fossil deposit/age. Germany: Messel Formation, Grube Messel Pit (type locality: grid square F11, 1.0 m above alpha); 48.6-40.4 Ma (Eocene).

Literature. Tröster (1991: 104): description, without formal name [62]; Tröster (1994: 148): original description [66]; Wappler (2003: 89): revision [69].

\section{- $\quad$ Genus Plagioraphes Iablokoff-Khnzorian, 1961}

Plagioraphes Iablokoff-Khnzorian, 1961: 84 [47]. Type species: Plagioraphes fasciatus Iablokoff-Khnzorian, 1961. For more information, see Kundrata et al. [12]. 


\section{Plagioraphes fasciatus Iablokoff-Khnzorian, 1961}

Plagioraphes fasciatus Iablokoff-Khnzorian, 1961: 85 [47]. (PIN).

Type material. Holotype, sex unknown, exoskeleton, amber inclusion, No. 364/346

Fossil deposit/age. Baltic amber; 38.0-33.9 Ma (Eocene).

Literature. Iablokoff-Khnzorian (1961: 85): original description [47]; Larsson (1978: 153): catalogue [48]; Spahr (1981: 49): catalogue [49]; Keilbach (1982: 246): catalogue [133]; Carpenter (1992: 305): generic catalogue [68]; Alekseev (2013: 7): checklist [92]; Kundrata et al. (2020: 5): generic catalogue [12].

\subsubsection{Tribe Cryptocardiini Dolin, 1980}

Cryptocardiini Dolin, 1980: 74 [53]. Type genus: Cryptocardius Dolin, 1980: 74 [53].

Remark. This group most probably does not belong to Agrypninae. Dolin [53] compared Cryptocardiini with Oophorini due to similar habitus, closed pronotosternal sutures, and structure of prosternum (without further details). He wrote that Cryptocardiini differ from Oophorini in the narrowed mesoventrite, less broadened metacoxal plates, and simple tarsi. However, Dolin [53] mentioned that the shape of antennae (i.e., antennomeres being shortened from antennomere VI to apex) and the cordate scutellar shield of Cryptocardius are not typical for Agrypninae at all. Drawings of Cryptocardius in Dolin [53] show that this click-beetle has enlarged pronotum which is sinuate near posterior angles, and has short basal furrows. Additionally, there is a short incision near basal furrow on the posterior angle of pronotum. These characters suggest that Cryptocardius might have been related to Hypnoidini.

\section{- Genus Cryptocardius Dolin, 1980}

Cryptocardius Dolin, 1980: 74 [53]. Type species: Cryptocardius mirabilis Dolin, 1980: 75 [53]. For more information, see Kundrata et al. [12].

\section{Cryptocardius mirabilis Dolin, 1980}

Cryptocardius mirabilis Dolin, 1980: 75 [53].

Type material. Holotype, sex unknown, exoskeleton, compression fossil, No. 2554/649 (part + counterpart) (PIN).

Fossil deposit/age. Kazakhstan: Karabastau Formation, Karatau, Mikhailovka; 166.1157.3 Ma (Jurassic).

Literature. Dolin (1980: 75): original description [53]; Carpenter (1992: 304): generic catalogue [68]; Korneev and Cate (2005: 10): checklist [120]; Alekseev (2011: 423): checklist [75]; Kundrata et al. (2020: 6): generic catalogue [12].

\subsubsection{Tribe Hemirhipini Candèze, 1857 *}

Hémirhipides Candèze, 1857: 199 [116]. Type genus: Hemirhipus Berthold, 1827: 336 [134]. For more information, including synonyms, see Kundrata et al. [109].

\section{- Genus Alaus Eschscholtz, 1829*}

Alaus Eschscholtz, 1829: 33 [117]. Type species: Elater oculatus Linnaeus, 1758: 404 [115]. For more information, including synonyms, see Kundrata et al. [109].

\section{Alaus spectabilis (Heer, 1865)}

Elater (Alaus) spectabilis Heer, 1865: 378 [17].

Alaus spectabilis: Handlirsch, 1907: 744 [127].

Type material. Holotype, sex unknown, compression fossil (UZH).

Fossil deposit/age. Germany: Upper Freshwater-Molasse Formation, Öhningen; 12.7-11.608 Ma (Miocene). 
Literature. Heer (1865: 378): original description, figure [17]; Heer (1870: 75): remark [18]; Heer (1872: 463): remark [135]; Heer (1876: 34): remark [136]; Heer (1883: 404): figure [137]; Scudder (1891: 518): catalogue [24]; Handlirsch (1907: 744): catalogue [127].

\subsubsection{Tribe Oophorini Gistel, 1848 *}

Oophoridae Gistel, 1848: 5 [138]. Type genus: Oophorus Dejean, 1833: 93 [139] (syn. of Aeolus Eschscholtz, 1829: 33 [117]). For more information, including synonyms, see Kundrata et al. [109].

\section{- Genus Monocrepidius Eschscholtz, 1829*}

Monocrepidius Eschscholtz, 1829: 31 [117]. Type species: Monocrepidius pallipes Eschscholtz, 1829: 32 [117]. For more information, including synonyms, see Kundrata et al. [109].

\section{Monocrepidius dubiosus Wickham, 1916}

Monocrepidius dubiosus Wickham, 1916: 508 [28].

Type material. Holotype, sex unknown, compression fossil, No. 90,483 (USNM).

Fossil deposit/age. USA: Colorado, Florissant Formation, Wilson Ranch; 37.2-33.9 Ma (Eocene).

Literature. Wickham (1916: 508): original description [28]; Wickham (1920: 354): catalogue [29].

\subsubsection{Tribe Pseudomelanactini Arnett, 1967 *}

Pseudomelanactini Arnett, 1967: 111 [140]. Type genus: Pseudomelanactes Mathieu, 1961: 474 [141] (synonym of Anthracalaus Fairmaire, 1888: 349 [142]).

\section{- Genus Lanelater Arnett, 1952 *}

Lanelater Arnett, 1952: 105 [143]. Type species: Agrypnus schotti LeConte, 1853: 492 [144]. For more information, including synonyms, see Kundrata et al. [109].

\section{Lanelater nicoleae Wappler, 2003}

Lanelater nicoleae Wappler, 2003: 90 [69].

Type material. Holotype, sex unknown, exoskeleton, compression fossil, PE_2000/349 $\mathrm{a}+\mathrm{b}$ LS (NHMM). Three paratypes, sex unknown, exoskeletons, compression fossils, PE_1994/79 a+b, LS, PE_1993/256 a+b, LS, PE_1992/79, LS (NHMM).

Fossil deposit/age. Germany: Eifel Formation, Eckfeld Maar; 48.6-40.4 Ma (Eocene).

Literature. Wappler (2003: 90): original description [69].

\section{Lanelater verae Tröster, 1993}

Lanelater verae Tröster, 1993: 51 [64].

Type material. Holotype, sex unknown, exoskeleton, compression fossil, SMF MeI 3735 (FIS). Five paratypes, sex unknown, exoskeletons, compression fossils, SMF MeI 1384, SMF MeI 1969, SMF MeI 1593, SMF MeI 1978, SMF MeI 226 (FIS).

Fossil deposit/age. Germany: Messel Formation, Grube Messel Pit (type locality: grid square H12, 0.00-0.26 m below horizon M); 48.6-40.4 Ma (Eocene).

Literature. Tröster (1993: 51): original description [64]; Wappler (2003: 92): remark [69].

\subsubsection{Tribe Pyrophorini Candèze, 1863 *}

Pyrophorites Candèze, 1863: 3 [145]. Type genus: Pyrophorus Billberg, 1820: 20 [146]. For more information, including synonyms, see Kundrata et al. [109]. 


\section{- Genus Eopyrophorus Haupt, 1950}

Eopyrophorus Haupt, 1950: 101 [44]. Type species: Eopyrophorus mixtus Haupt, 1950: 107 [44]. For more information, see Kundrata et al. [12].

Remark. Although this genus might indeed belong to Pyrophorini, its placement needs further examination since important tribal characters were not discussed by Haupt [44].

\section{Eopyrophorus mixtus Haupt, 1950}

Eopyrophorus mixtus Haupt, 1950: 107 [44].

Type material. Type, sex unknown, compression fossil (GIH).

Fossil deposit/age. Germany: Geiseltal; 47.8-41.3 Ma (Eocene).

Literature. Haupt (1950: 107): original description [44]; Haupt (1956: 48): catalogue [147]; Carpenter (1992: 304): generic catalogue [68]; Kundrata et al. (2020: 6): generic catalogue [12].

\subsection{Subfamily Cardiophorinae Candèze, 1859 *}

Cardiophorites Candèze, 1859: 4 [148]. Type genus: Cardiophorus Eschscholtz, 1829: 34 [117]. For more information, including synonyms, see Bouchard et al. [110] and Douglas [108].

Remark. Type material of most species should be examined in order to confirm their placement in Cardiophorinae. Some species may represent Negastriinae. Considering the current limits and diagnoses of cardiophorine genera, it is impossible for most species to assign them to a proper genus based on available characters [108].

\section{- Genus Cardiophorus Eschscholtz, 1829*}

Cardiophorus Eschscholtz, 1829: 34 [117]. Type species: Elater thoracicus Fabricius, 1801: 236 [149] (synonym of Cardiophorus gramineus (Scopoli, 1763: 95) [150]). For more information, see Douglas [108].

\section{Cardiophorus braunii Heer, 1847}

Cardiophorus braunii Heer, 1847: 134 [14].

Cardiophorus brauni: Giebel (1852: 651) [126] [unavailable name, incorrect subsequent spelling not in prevailing usage; [129], Art. 33.4].

Type material. Holotype, sex unknown, compression fossil (SMNK).

Fossil deposit/age. Germany: Upper Freshwater-Molasse Formation, Öhningen; 12.7-11.608 Ma (Miocene).

Literature. Heer (1847: 134): original description [14]; Giebel (1852: 651): catalogue [126]; Giebel (1856: 97): redescription [16]; Scudder (1891: 486): catalogue [24]; Handlirsch (1907: 746): catalogue [127]; Cockerell (1926: 10): comparison with other species [35].

\section{Cardiophorus cockerelli Wickham, 1916}

Cardiophorus cockerelli Wickham, 1916: 503 [28].

Type material. Holotype, sex unknown, exoskeleton, compression fossil, MCZ 2765 (=1916 Scudder coll.) (MCZ).

Fossil deposit. USA: Colorado, Florissant Formation, Florissant; 37.2-33.9 Ma (Eocene).

Literature. Wickham (1916: 503): original description [28]; Wickham (1920: 354): catalogue [29].

\section{Cardiophorus deprivatus Wickham, 1916}

Cardiophorus deprivatus Wickham, 1916: 504 [28].

Type material. Holotype, sex unknown, compression fossil, No. 8206 (99/127) (CUB).

Fossil deposit/age. USA: Colorado, Florissant Formation, Chadronian, Station 13; 37.2-33.9 Ma (Eocene). 
Literature. Wickham (1916: 504): original description [28]; Wickham (1920: 354): catalogue [29].

\section{Cardiophorus exhumatus Cockerell, 1926}

Cardiophorus exhumatus Cockerell, 1926: 9 [35].

Type material. Holotype, sex unknown, exoskeleton, compression fossil, No. 69614 (USNM).

Fossil deposit/age. USA: Colorado, Green River Formation, head of East Alkali Creek, approximately 8 miles south of De Beque; 50.3-46.2 Ma (Eocene).

Literature. Cockerell (1926: 9): original description [35]; Wickham (1927: 55): catalogue [151].

\section{Cardiophorus florissantensis Wickham, 1916}

Cardiophorus florissantensis Wickham, 1916: 502 [28].

Type material. Holotype, sex unknown, compression fossil, No. 8205 (CUB).

Fossil deposit/age. USA: Colorado, Florissant Formation, Florissant, Station 13; 37.233.9 Ma (Eocene).

Literature. Wickham (1916: 502): original description [28]; Wickham (1920: 354): catalogue [29].

\section{Cardiophorus lithographus Wickham, 1916}

Cardiophorus lithographus Wickham, 1916: 501 [28].

Type material. Holotype, sex unknown, compression fossil, 90,611 (USNM).

Fossil deposit/age. USA: Colorado, Florissant Formation, Wilson Ranch; 37.2-33.9 Ma (Eocene).

Literature. Wickham (1916: 401): original description [28]; Cockerell (1926: 10): comparison with other species [29].

\section{Cardiophorus requiescens Wickham, 1916}

Cardiophorus requiescens Wickham, 1916: 504 [28].

Type material. Holotype, sex unknown, compression fossil, 90,612 (USNM).

Fossil deposit/age. USA: Colorado, Florissant Formation, Wilson Ranch; 37.2-33.9 Ma (Eocene).

Literature. Wickham (1916: 504): original description [28]; Wickham (1920: 354): catalogue [29].

\section{Cardiophorus yatsenkokhmelevskyi Iablokoff-Khnzorian, 1961}

Cardiophorus yatsenkokhmelevskyi Iablokoff-Khnzorian, 1961: 94 [47].

Type material. Holotype, sex unknown, exoskeleton, amber inclusion, No. 364/656 (PIN).

Fossil deposit/age. Baltic amber; 38.0-33.9 Ma (Eocene).

Literature. Iablokoff-Khnzorian (1961: 94): original description [47]; Larsson (1978: 153): catalogue [48]; Spahr (1981: 46): catalogue [49]; Keilbach (1982: 246): catalogue [133]; Hawkeswood et al. (2009: 189): catalogue [105]; Alekseev (2013: 7): checklist [92].

\section{- Genus Horistonotus Candèze, 1860 *}

Horistonotus Candèze, 1860: 243 [152]. Type species: Horistonotus flavidus Candèze, 1860: 250 [152]. For more information, see Douglas [108].

\section{Horistonotus coloradensis Wickham, 1916}

Horistonotus coloradensis Wickham, 1916: 505 [28].

Type material. Holotype, sex unknown, compression fossil, No. 90,547 (USNM). Fossil deposit/age. USA: Colorado, Florissant Formation; 37.2-33.9 Ma (Eocene). 
Literature. Wickham (1916: 505): original description [28]; Wickham (1920: 354): catalogue [29].

\subsection{Subfamily Dendrometrinae Gistel, 1848 *}

Dendrometridae Gistel, 1848: 5 [138]. Type genus: Dendrometrus Gistel, 1848: 5 [138]. For more information, including synonyms, see Bouchard et al. [110].

\subsubsection{Tribe Dendrometrini Gistel, 1848 *}

Dendrometridae Gistel, 1848: 5 [138]. Type genus: Dendrometrus Gistel, 1848: 5 [138]. For more information, including synonyms, see Bouchard et al. [110].

\section{- Genus Athous Eschscholtz, 1829*}

Athous Eschscholtz, 1829: 33 [117]. Type species: Elater vittatus Fabricius, 1792: 224 [153]. For more information, including synonyms, see Sánchez-Ruiz [121] and Cate [107].

\section{Subgenus Athousiomorphus Iablokoff-Khnzorian, 1961}

Athousiomorphus Iablokoff-Khnzorian, 1961: 92 [47]. Type species: Athous (Athousiomorphus) olgae Iablokoff-Khnzorian, 1961: 92 [47]. For more information, see Kundrata et al. [12].

\section{Athous (Athousiomorphus) olgae Iablokoff-Khnzorian, 1961}

Athous (Athousiomorphus) olgae Iablokoff-Khnzorian, 1961: 92 [47].

Athous olgae Larsson, 1978: 153 [48].

Type material. Holotype, male, exoskeleton, amber inclusion, No. 364/655 (PIN).

Fossil deposit/age. Baltic amber; 38.0-33.9 Ma (Eocene).

Literature. Iablokoff-Khnzorian (1961: 92): original description [47]; Larsson (1978: 153): catalogue [48]; Spahr (1981: 46): catalogue [49]; Keilbach (1982: 246): catalogue [133]; Schimmel (2005: 27): remark [91]; Alekseev (2013: 7): checklist [92]; Kundrata et al. (2020: 7): generic catalogue [12].

\section{Subgenus incertae sedis}

\section{Athous contusus Wickham, 1916}

Athous contusus Wickham, 1916: 519 [28].

Type material. Holotype, sex unknown, exoskeleton, compression fossil, MCZ 2727 (=8346 in Scudder coll.) (MCZ).

Fossil deposit/age. USA: Colorado, Florissant Formation, Florissant; 37.2-33.9 Ma (Eocene).

Literature. Wickham (1916: 519): original description [28]; Wickham (1920: 354): catalogue [29].

\section{Athous fractus Wickham, 1916}

Athous fractus Wickham, 1916: 519 [28].

Type material. Holotype, sex unknown, compression fossil, No. 8240 (CUB).

Fossil deposit/age. USA: Colorado, Florissant Formation, Florissant, Station 14; 37.233.9 Ma (Eocene).

Literature. Wickham (1916: 519): original description [28]; Wickham (1920: 354): catalogue [29].

Remark. Based on the body size, general habitus, structure of antenna and thorax, this species might belong to Prosternini or Selatosomini. Most striking character which challenges the position of this species in Athous is the pronotum, which is rather broad, more or less rounded at sides and sinuate near posterior angles (usually parallel sided and rather narrowed in Athous), and with each posterior angle bearing a sublateral carina (not 
typical for Athous). We prefer to postpone any taxonomic changes pending a comprehensive review including the type material.

\section{Athous holmgreni (Heer, 1870)}

Elater holmgreni Heer, 1870: 75 [18].

Athous holmgreeni: Birket-Smith, 1977: 18 [154] [unavailable name, incorrect subsequent spelling not in prevailing usage; [129], Art. 33.3].

Type material. Holotype, sex unknown, elytron, compression fossil, No. 53 (SMNH).

Fossil deposit/age. Norway: Svalbard and Jan Mayen, Firkanten Formation, Cap Staratschin; 66.0-59.2 Ma (Paleocene).

Literature. Heer (1870: 75): original description [18]; Scudder (1891: 517): catalogue [24]; Handlirsch (1907: 745): catalogue [127]; Birket-Smith (1977: 17): taxonomic remark [154]; Tröster (1994: 39): remark [65].

Remark. The description of this species was based on a part of isolated elytron and, therefore, its generic attribution is rather problematic.

\section{Athous lethalis Wickham, 1916}

Athous lethalis Wickham, 1916: 518 [28].

Type material. Holotype, sex unknown, exoskeleton, compression fossil, MCZ 2728 and 2729 (=8464 and 8713 in Scudder coll.) (MCZ).

Fossil deposit. USA: Colorado, Florissant Formation, Florissant; 37.2-33.9 Ma (Eocene).

Literature. Wickham (1916: 518): original description [28]; Wickham (1920: 354): catalogue [29].

\section{- Genus Limonius Eschscholtz, 1829 *}

Limonius Eschscholtz, 1829: 33 [117]. Type species: Elater minutus Linnaeus, 1758: 406 [115]. For more information, including synonyms, see Sánchez-Ruiz [121], Cate [107] and Etzler [155].

\section{Subgenus Paralimonius Iablokoff-Khnzorian, 1961}

Paralimonius Iablokoff-Khnzorian, 1961: 91 [47]. Type species: Limonius (Paralimonius) barovskyi Iablokoff-Khnzorian, 1961: 91 [47]. For more information, see Kundrata et al. [12].

\section{Limonius (Paralimonius) barovskyi Iablokoff-Khnzorian, 1961}

Limonius (Paralimonius) barovskyi Iablokoff-Khnzorian, 1961: 91 [47].

Limonius barovskyi: Larsson, 1978: 153 [48].

Type material. Holotype, sex unknown, exoskeleton, amber inclusion, No. 364/654 (PIN).

Fossil deposit/age. Baltic amber; 38.0-33.9 Ma (Eocene).

Literature. Iablokoff-Khnzorian (1961: 91): original description [47]; Larsson (1978: 153): catalogue [48]; Spahr (1981: 48): catalogue [49]; Keilbach (1982: 246): catalogue [133]; Schimmel (2005: 27): remark [91]; Alekseev (2013: 7): checklist [92]; Kundrata et al. (2020: 7): generic catalogue [12].

\section{Subgenus incertae sedis}

\section{Limonius aboriginalis Wickham, 1916}

Limonius aboriginalis Wickham, 1916: 514 [28].

Type material. Holotype, sex unknown, compression fossil, No. 90,474 (USNM).

Fossil deposit/age. USA: Colorado, Florissant Formation, Wilson Ranch, Florissant; 37.2-33.9 Ma (Eocene).

Literature. Wickham (1916: 514): original description [28]; Wickham (1920: 354): catalogue [29]. 
Remark. Representatives of Limonius and related genera have more elongated elytra and more or less campaniform or parallel-sided pronotum, whereas this species has elytra only slightly elongate, and the pronotum arcuate at sides and sinuate near posterior angles. Such body proportions and shape of thorax are usually found in Cardiophorinae and Negastriinae. Based on the image of prothorax [28], L. aboriginalis is most probably a member of Cardiophorinae; its prosternum is narrow and prosternal sutures almost parallel sided while in Negastriinae the prosternum is rather broad and prosternal sutures curved outward. However, we prefer to postpone any taxonomic changes pending a comprehensive review including the type material.

\section{Limonius florissantensis Wickham, 1916}

Limonius florissantensis Wickham, 1916: 515 [28].

Type material. Two syntypes (one with counterpart), sex unknown, compression fossils, No. 90,473 (USNM).

Fossil deposit/age. USA: Colorado, Florissant Formation, Wilson Ranch, Florissant; 37.2-33.9 Ma (Eocene).

Literature. Wickham (1916: 515): original description [28]; Wickham (1920: 354): catalogue [29].

Remark. Representatives of Limonius and related genera have more elongated elytra and more or less campaniform or parallel-sided pronotum, whereas this species has elytra only slightly elongate, and the pronotum arcuate at sides and sinuate near posterior angles. Such body proportions and shape of thorax are usually found in Cardiophorinae and Negastriinae. Based on the prothorax image in Wickham [28], L. florissantensis is most probably a member of Negastriinae as its pronotum is less globular than in typical Cardiophorinae. However, the type material should be thoroughly examined before any taxonomic change can be made.

\section{Limonius impunctus Scudder, 1895}

Limonius impunctus Scudder, 1895: 37 [156].

Type material. Holotype, sex unknown, elytron, compression fossil, No. 100a, b (GSC).

Fossil deposit/age. Canada: Allenby Formation (Princeton Group), North Fork of Similkameen River; 56.0-47.8 Ma (Eocene).

Literature. Scudder (1895: 37): original description [156]; Scudder (1900: 96): catalogue [157]; Handlirsch (1907: 746): catalogue [127]; Wickham (1920: 354): catalogue [29].

Remark. The generic attribution of this species is unclear as it was described based only on elytral characters.

\section{Limonius optabilis Heer, 1847}

Limonius optabilis Heer, 1847: 137 [14].

Type material. Holotype, sex unknown, compression fossil (ETH).

Fossil deposit/age. Germany: Upper Freshwater-Molasse Formation, Öhningen, Upper Öhningen beds; 12.7-11.608 Ma (Miocene).

Literature. Heer (1847: 137): original description [14]; Giebel (1852: 651): catalogue [126]; Giebel (1856: 95): redescription [16]; Heyden (1862: 69): remark [158]; Scudder (1891: 547): catalogue [24]; Handlirsch (1907: 746): catalogue [127].

Remark. Generic attribution of this species is doubtful. It might belong to Agrypnini based on the body proportions and the structure of thorax, especially as figured in Figure $6 \mathrm{~b}$ in Heer [14]. Note, that drawing in Figure $6 \mathrm{c}$ in Heer [14] differs considerably in many features from Figure $6 b$ in the same study.

\section{Limonius praecursor Wickham, 1916}

Limonius praecursor Wickham, 1916: 516 [28].

Type material. Holotype, sex unknown, exoskeleton, compression fossil, MCZ 2730 and 2731 (=9417 and 10,558 in Scudder coll.) (MCZ). 
Fossil deposit/age. USA: Colorado, Florissant Formation, Florissant; 37.2-33.9 Ma (Eocene).

Literature. Wickham (1916: 516): original description [28]; Wickham (1920: 354): catalogue [29].

\section{Limonius shoshonis Wickham, 1916}

Limonius shoshonis Wickham, 1916: 517 [28].

Type material. Holotype, sex unknown, compression fossil, No. 8251 (58) (CUB).

Fossil deposit/age. USA: Colorado, Florissant Formation, Florissant, Station 14; 37.233.9 Ma (Eocene).

Literature. Wickham (1916: 517): original description [28]; Wickham (1920: 354): catalogue [29].

Remark. Representatives of Limonius and related genera have more elongated elytra and more or less campaniform or parallel-sided pronotum, whereas this species has elytra only slightly elongate, and the pronotum arcuate at sides and sinuate near posterior angles. Such body proportions and shape of thorax are usually found in Cardiophorinae and Negastriinae. Based on the prothorax image in Wickham [28], L. shoshonis is most probably a member of Negastriinae as its pronotum is less globular than in typical Cardiophorinae. However, the type material should be thoroughly examined before any taxonomic change can be made.

\section{Limonius volans Wickham, 1916}

Limonius volans Wickham, 1916: 517 [28].

Type material. Holotype, sex unknown, compression fossil, No. 8252 (CUB).

Fossil deposit/age. USA: Colorado, Florissant Formation, Florissant, Station 14; 37.2 33.9 Ma (Eocene).

Literature. Wickham (1916: 517): original description [28]; Wickham (1920: 354): catalogue [29].

\subsubsection{Tribe Dimini Candèze, 1863 *}

Dimites Candèze, 1863: 237 [145]. Type genus: Dima Charpentier, 1825: 191 [159]. For more information, see Kundrata et al. [12,160].

\section{- Genus Alaodima Dolin, 1980}

Alaodima Dolin, 1980: 75 [53]. Type species: Alaodima grandis Dolin, 1980: 76 [53]. For more information, see Kundrata et al. [12].

Remark. We keep this genus tentatively under Dimini although Schimmel [91] and Schimmel and Tarnawski [131] placed it to Elaterinae without any explanation. Some characters of $A$. grandis, e.g., large body size, pointed last ventrite, attenuate elytral apices, transverse scutellar shield, are more typical for species of Oxynopterini rather than Dimini, though $A$. grandis differs from Oxynopterini in the structure of metacoxal plate and the shape of prosternal process. Unfortunately, this fossil lacks head and legs so many crucial diagnostic characters are absent.

\section{Alaodima grandis Dolin, 1980}

Alaodima grandis Dolin, 1980: 76 [53].

Type material. Holotype, sex unknown, exoskeleton, compression fossil, No. 2066/2970 (part + counterpart) (PIN).

Fossil deposit/age. Kazakhstan: Karabastau Formation, Karatau, Mikhailovka; 166.1157.3 Ma (Jurassic).

Literature. Dolin (1980: 76): original description [53]; Carpenter (1992: 304): generic catalogue [68]; Korneev and Cate (2005: 9): checklist [120]; Schimmel (2005: 28): remark, photo [91]; Schimmel and Tarnawski (2010: 363): remark [131]; Kundrata et al. (2018: 69): 
catalogue [160]; Kundrata et al. (2020: 7): generic catalogue [12]; Kundrata et al. (2020: 8): remark [94].

\subsubsection{Tribe Hypnoidini Schwarz, 1906 *}

Hypnoidini Schwarz, 1906: 150 [161]. Type genus: Hypnoidus Dillwyn, 1829: 32 [162]. For more information, including synonyms, see Bouchard et al. [110].

\section{- Genus Ligmargus Stibick, 1976 *}

Ligmargus Stibick, 1976: 210 [163]. Type species: Cryptohypnus funebris Candèze, 1860: 62 [152]. For more information, see Cate [107].

\section{Ligmargus terrestris (Scudder, 1879)}

Cryptohypnus terrestris Scudder, 1879: 180 [20].

Ligmargus terrestris: Stibick, 1981: 247 [164].

Type material Holotype, sex unknown, compression fossil, No. 59 (GSC).

Fossil deposit/age. Canada: British Columbia, Princeton Group, Nicola river; 56.047.8 Ma (Eocene).

Literature. Scudder (1879: 180): original description [20]; Scudder (1890: 497): catalogue [165]; Scudder (1891: 503): catalogue [24]; Scudder (1895: 38): catalogue [156]; Scudder (1900: 96): catalogue [157]; Handlirsch (1907: 745): catalogue [127]; Wickham (1920: 354): catalogue [29]; Stibick (1981: 247): revision [164].

\subsubsection{Tribe Oxynopterini Candèze, $1857^{*}$}

Oxynopterini Candèze, 1857: 355 [116]. Type genus: Oxynopterus Hope, 1842: 77 [166]. For more information, including synonyms, see Bouchard et al. [110].

\section{- Genus Campsosternus Latreille, 1834 *}

Campsosternus Latreille, 1834: 141 [167]. Type species: Elater fulgens Olivier, 1790: 12 [168] (syn. of Elater auratus Drury, 1773: 65 [169]). For more information, see Cate [107].

\section{Campsosternus atavus Deichmüller, 1881}

Campsosternus atavus Deichmüller, 1881: 306 [21].

Type material. Unknown number of type specimens, probably only one, sex unknown, compression fossil (type depository unknown).

Fossil deposit/age. Czech Republic: Kučlín (u Bíliny); 37.2-33.9 Ma (Eocene).

Literature. Deichmüller (1881: 306): original description [21]; Scudder (1891: 483): catalogue [24]; Handlirsch (1907: 743): catalogue [127].

\section{- Genus Melanactes LeConte, 1853 *}

Melanactes LeConte, 1853: 493 [144]. Type species. Melanactes densus LeConte, 1853: 494 [144]. For more information, see Mathieu [141].

\section{Melanactes cockerelli Wickham, 1908}

Melanactes cockerelli Wickham, 1908: 77 [27].

Type material. Holotype, sex unknown, exoskeleton, compression fossil, No. 3 (YPM).

Fossil deposit/age. USA: Colorado, Florissant Formation, Florissant, Station 14; $37.2-$ 33.9 Ma (Eocene).

Literature. Wickham (1908: 77): original description [27]; Wickham (1916: 527): catalogue [28]; Wickham (1920: 354): catalogue [29]. 


\subsubsection{Tribe Prosternini Gistel, 1856 *}

Prosternidae Gistel, 1856: 367 [170]. Type genus: Prosternon Latreille, 1834: 151 [167]. This tribe includes also Ctenicerini Jakobson, 1913 and Corymbitini LeConte, 1861, which both have been currently synonyms of Prosternini. For more information, see Cate [107] and Bouchard et al. [110].

\section{- Genus Ctenicera Latreille, 1829 *}

Ctenicera Latreille, 1829: 454 [118]. Type species: Elater pectinicornis Linnaeus, 1758: 406 [115]. This genus includes also species earlier attributed to genera Ludius Eschscholtz, 1829 (nec Berthold [134], nec Latreille [167]) and Corymbites Latreille, 1834, which are currently both synonyms of Ctenicera. For more information, see Hyslop [111] and Cate [107].

Remark. The generic assignment of all species classified under Ctenicera needs serious re-examination. Many of them probably belong to Selatosomini.

Ctenicera emblemoelytra (Zhang, 1989), comb. nov.

Corymbites emblemoelytrus Zhang, 1989: 125 [58].

Type material. Holotype, sex unknown, exoskeleton, compression fossil, 750115/750116 (part + counterpart) (SMJS).

Fossil deposit/age. China: Shanwang Formation, Linqu County; 20.44-15.97 Ma (Miocene).

Literature. Zhang (1989: 125): original description [58]; Dong and Huang (2011: 1225): checklist [81].

Remark. This species was described in genus Corymbites, which is, however, a synonym of Ctenicera [107,111]. Generic attribution of this species remains unclear. Since it was compared with a recent species of Pristilophus Latreille, 1834 and with fossil C. primitiva (Wickham, 1908) [58], which might in fact belong to Selatosomus Stephens, 1830, affinities of this species to the tribe Selatosomini should be taken into consideration.

Ctenicera euprepes (Zhang, Sun and Zhang, 1994), comb. nov.

Corymbites euprepes Zhang, Sun and Zhang, 1994: 92 [59].

Type material. Holotype, sex unknown, compression fossil, K0253 (SGMS).

Fossil deposit/age. China: Shanwang Formation, Linqu County; 20.44-15.97 Ma (Miocene).

Literature. Zhang et al. (1994: 92): original description [59]; Dong and Huang (2011: 1225): checklist [81].

Remark. This species was described in genus Corymbites, which is, however, a synonym of Ctenicera $[107,111]$. Its generic placement is unclear, since it superficially resembles the representatives of tribe Selatosomini.

Ctenicera granulicollis (Wickham, 1908), comb. nov.

Corymbites granulicollis Wickham, 1908: 76 [27].

Ludius granulicollis: Wickham, 1920: 354 [29].

Type material. Holotype, sex unknown, exoskeleton, compression fossil, No. 1 (YPM). Fossil deposit/age. USA: Colorado, Florissant Formation, Florissant Station 14; 37.2 33.9 Ma (Eocene).

Literature. Wickham (1908: 76): original description [27]; Wickham (1916: 524): catalogue [28]; Wickham (1920: 354): catalogue [29]; Zhang et al. (1994: 93): remark [59].

Remark. This species was described in genus Corymbites and later transferred to Ludius Eschscholtz, 1829 (nec Berthold [134], nec Latreille [167]) [29] which are currently both synonyms of Ctenicera $[107,111]$. This species most probably belongs to genus Selatosomus based on the body proportions and shapes of prothorax and elytra. However, we prefer to postpone any taxonomic changes pending a detailed examination of the type material. 
Ctenicera primitiva (Wickham, 1908), comb. nov.

Corymbites primitious Wickham, 1908: 77 [27].

Ludius primitivus: Wickham, 1920: 354 [29].

Type material. Holotype, sex unknown, exoskeleton, compression fossil, No. 2 (YPM).

Fossil deposit/age. USA: Colorado, Florissant Formation, Florissant Station 13; 37.233.9 Ma (Eocene).

Literature. Wickham (1908: 77): original description [27]; Wickham (1916: 524): catalogue [28]; Wickham (1920: 354): catalogue [29]; Zhang (1989: 126): remark [58].

Remark. This species was described in genus Corymbites and later transferred to Ludius Eschscholtz, 1829 (nec Berthold [134], nec Latreille [167]) [29] which are currently both synonyms of Ctenicera $[107,111]$. This species most probably belongs to genus Selatosomus based on the body proportions and shapes of prothorax and elytra. However, we prefer to postpone any taxonomic changes pending a detailed examination of the type material.

Ctenicera prophetica (Wickham, 1916), comb. nov.

Corymbites propheticus Wickham, 1916: 526 [28].

Ludius propheticus: Wickham, 1920: 354 [29].

Type material. Holotype, sex unknown, exoskeleton, compression fossil, MCZ 2724 (=13,657 in Scudder coll.) (MCZ). (Eocene).

Fossil deposit/age. USA: Colorado, Florissant Formation, Florissant; 37.2-33.9 Ma

Literature. Wickham (1916: 526): original description [28]; Wickham (1920: 354): catalogue [29].

Remark. This species was described in genus Corymbites and later transferred to Ludius Eschscholtz, 1829 (nec Berthold [134], nec Latreille [167]) [29] which are currently both synonyms of Ctenicera $[107,111]$. This species superficially resembles Selatosomini but its placement remains unclear.

Ctenicera restructa (Wickham, 1916), comb. nov.

Corymbites restructus Wickham, 1916: 525 [28].

Ludius restructus: Wickham, 1920: 354 [29].

Type material. Holotype, sex unknown, compression fossil, No. 8215 (CUB).

Fossil deposit. USA: Colorado, Florissant Formation, Florissant, Station 14; 37.2-33.9 Ma (Eocene).

Literature. Wickham (1916: 525): original description [28]; Wickham (1920: 354): catalogue [29].

Remark. This species was described in genus Corymbites and later transferred to Ludius Eschscholtz, 1829 (nec Berthold [134], nec Latreille [167]) [29] which are currently both synonyms of Ctenicera $[107,111]$. This species superficially resembles Selatosomini but its placement remains unclear.

Ctenicera sincera (Zhang, Sun and Zhang, 1994), comb. nov.

Corymbites sincerus Zhang, Sun and Zhang, 1994: 92 [59].

Type material. Holotype, sex unknown, compression fossil, SK000434 (Shanwang Fossil Protection Post collection; information taken from the Paleobiology Database, https:/ / paleobiodb.org, accessed on 10 October 2020. May be "Shanwang Palaeontological Museum" in Linqu County (L. Qiu, personal communication). We have not been able to confirm depository information).

Fossil deposit/age. China: Shanwang Formation, Linqu County; 20.44-15.97 Ma (Miocene).

Literature. Zhang et al. (1994: 92): original description [59]; Dong and Huang (2011: 1225): checklist [81]. 
Remark. This species was described in genus Corymbites, which is currently a synonym of Ctenicera [107,111]. Generic attribution of this species remains unclear.

Ctenicera submersa (Wickham, 1916), comb. nov.

Corymbites submersus Wickham, 1916: 524 [28].

Ludius submersus: Wickham, 1920: 354 [29].

Type material. Holotype, sex unknown, compression fossil, No. 8216 (CUB).

Fossil deposit/age. USA: Colorado, Florissant Formation, Florissant, Station 14; 37.233.9 Ma (Eocene).

Literature. Wickham (1916: 524): original description [28]; Wickham (1920: 354): catalogue [29].

Remark. This species was described in genus Corymbites and later transferred to Ludius Eschscholtz, 1829 (nec Berthold [134], nec Latreille [167]) [29] which are currently both synonyms of Ctenicera $[107,111]$. This species superficially resembles Oxynopterini but its placement remains unclear.

Ctenicera sutor (Heer, 1847), comb. nov.

Diacanthus sutor Heer, 1847: 136 [14].

Corymbites sutor: Heer, 1861: 204 [171]. 7911)).

Type material. Two syntypes, sex unknown, compression fossils (SMNK, ETH (No.

Fossil deposit/age. Germany: Upper Freshwater-Molasse Formation, Öhningen, MN 7 mammal zone; 12.7-11.608 Ma (Miocene).

Literature. Heer (1847: 136): original description [14]; Giebel (1852: 651): catalogue [126]; Giebel (1856: 95): revision, redescription [16]; Heer (1861: 204): catalogue [171]; Scudder (1891: 508): catalogue [24]; Handlirsch (1907: 746): catalogue [127]; Theobald (1937: 175): remark [42].

Remark. This species was classified in genus Corymbites [171] which is currently a synonym of Ctenicera $[107,111]$. This species is superficially similar to some species of Limonius but its placement remains unclear.

Ctenicera velata (Scudder, 1876), comb. nov.

Corymbites velatus Scudder, 1876: 81 [19].

Ludius velatus: Wickham, 1920: 354 [29].

Type material. Holotype, sex unknown, compression fossil, No. 3458 (MCZ).

Fossil deposit/age. USA: Wyoming, Green River Formation, Laney Member, Petrified fish cut; 50.3-46.2 Ma (Eocene).

Literature. Scudder (1876: 81): original description [19]; Scudder (1878: 762): catalogue [172]; Scudder (1890: 496): catalogue [165]; Scudder (1891: 501): catalogue [24]; Scudder (1900: 96): catalogue [157]; Handlirsch (1907: 746): catalogue [127]; Wickham (1920: 354): catalogue [29].

Remark. This species was described in genus Corymbites and later transferred to Ludius Eschscholtz, 1829 (nec Berthold [134], nec Latreille [167]) [29] which are currently both synonyms of Ctenicera $[107,111]$. The generic attribution of this species is unclear since it was described based almost exclusively on elytral characters.

\section{- Genus Eanus LeConte, 1861 *}

Eanus LeConte, 1861: 171 [173]. Type species: Limonius estriatus LeConte, 1853: 434 [144]. This genus includes also species earlier attributed to genus Paranomus Kiesenwetter, 1858 (earlier also as part of Ludius Eschscholtz, 1829, nec Berthold [134], nec Latreille [167]), which is currently a synonym of Eanus. For more information, see Hyslop [111], Johnson [106] and Cate [107].

Remark. All fossil species listed under this genus should be re-examined as their body proportions and structure of prothorax resemble more Negastriinae or Cardiophorinae 
rather than Eanus. Current representatives of Eanus have more elongated elytra and more or less campaniform pronotum, while in the here listed fossil species, elytra are relatively shorter and the pronotum is more or less rounded and sinuate near posterior angles. The shapes of scutellar shields in drawings by Wickham [28] also suggest similarity with Negastriinae.

Eanus exanimatus (Wickham, 1916), comb. nov.

Paranomus exanimatus Wickham, 1916: 520 [28].

Ludius exanimatus: Wickham, 1920: 354 [29].

Type material. Holotype, sex unknown, compression fossil, No. 90,496 (USNM).

Fossil deposit/age. USA: Colorado, Florissant Formation, Wilson Ranch, Florissant; 37.2-33.9 Ma (Eocene).

Literature. Wickham (1916: 520): original description [28]; Wickham (1920: 354): catalogue [29].

Remark. This species was described in genus Paranomus (earlier also as part of Ludius Eschscholtz, 1829, nec Berthold [134], nec Latreille [167]), which is currently a synonym of Eanus.

Eanus heeri (Wickham, 1916), comb. nov.

Paranomus heeri Wickham, 1916: 521 [28].

Ludius heeri: Wickham, 1920: 354 [29].

Type material. Holotype, sex unknown, compression fossil (?CUB).

Fossil deposit/age. USA: Colorado: Florissant Formation, Florissant, Station 14; 37.2-33.9 Ma (Eocene).

Literature. Wickham (1916: 521): original description [28]; Wickham (1920: 354): catalogue [29].

Remark. This species was described in genus Paranomus (later also as part of Ludius Eschscholtz, 1829, nec Berthold [134], nec Latreille [167]), which is currently a synonym of Eanus. Its generic placement is unclear, and already Wickham [28] mentioned that this was probably not true Paranomus (now Eanus).

Eanus laevissimus (Wickham, 1916), comb. nov.

Paranomus laevissimus Wickham, 1916: 521 [28].

Ludius laevissimus: Wickham, 1920: 354 [29].

Type material. Holotype, sex unknown, compression fossil (?CUB).

Fossil deposit/age. USA: Colorado, Florissant Formation, Florissant, Station 14; 37.2 33.9 Ma (Eocene).

Literature. Wickham (1916: 521): original description [28]; Wickham (1920: 354): catalogue [29].

Remark. This species was described in genus Paranomus (earlier also as part of Ludius Eschscholtz, 1829, nec Berthold [134], nec Latreille [167]), which is currently a synonym of Eanus.

\section{- Genus Oxygonus LeConte, 1863 *}

Oxygonus LeConte, 1863: 48 [174]. Type species: Elater obesus Say, 1823: 168 [175]. For more information, see Hyslop [111] and Johnson [106].

Oxygonus mortuus Scudder, 1876

Oxygonus mortuus Scudder, 1876: 81 [19].

Type material. Holotype, sex unknown, elytron, compression fossil (type depository has not been identified).

Fossil deposit/age. USA: Utah, Green River Formation, Fossil Cañon; 50.3-46.2 Ma (Eocene). 
Literature. Scudder (1876: 81): original description [19]; Scudder (1877: 759): catalogue [176]; Scudder (1890: 496): catalogue [165]; Scudder (1891: 562): catalogue [24]; Scudder (1900: 97): catalogue [157]; Handlirsch (1907: 747): catalogue [127]; Wickham (1920: 354): catalogue [29].

Remark. The generic attribution of this species is unclear as it was described based only on elytral characters.

\section{Oxygonus primus Wickham, 1916}

Oxygonus primus Wickham, 1916: 526 [28].

Type material. Holotype, sex unknown, exoskeleton, compression fossil, No. 4069 (=6381 in Scudder coll.) (MCZ) (Figure 1A).

Fossil deposit/age. USA: Colorado, Florissant Formation, Florissant; 37.2-33.9 Ma (Eocene).

Literature. Wickham (1916: 526): original description [28]; Wickham (1920: 354): catalogue [29].

Remark. This species most probably does not belong to Oxygonus as it differs considerably in the shape of prothorax (almost globular versus more or less elongate, with arcuate sides in Oxygonus) [177].

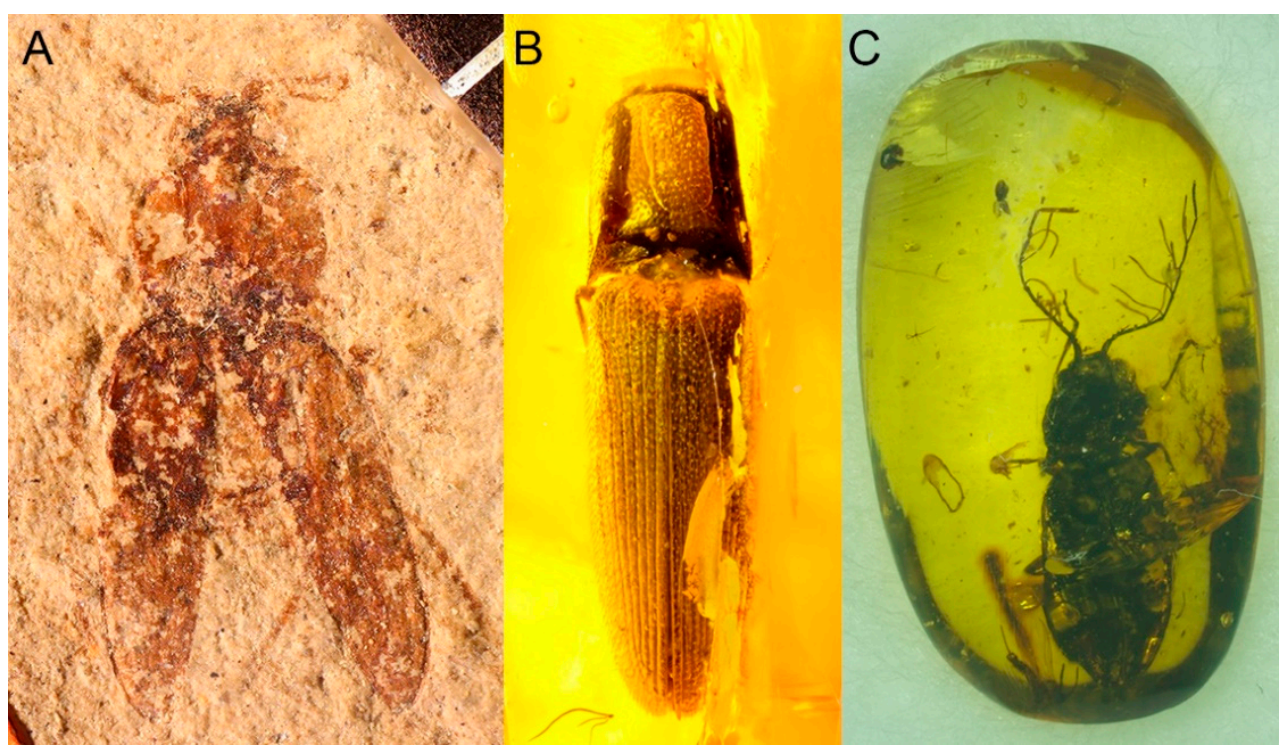

Figure 1. Type specimens of fossil Elateridae. (A) Oxygonus primus Wickham, 1916, holotype (MCZ), Florissant, Colorado, USA, body length: $6.0 \mathrm{~mm}$ (credit: MCZ-H. Meyer, M. Aja); (B) Megapenthes voigti Schimmel, 2005, paratype (BMNH), North European Baltic amber, body length: 5.9 mm (credit: BMNH-K. Matsumoto); (C) Cretopityobius pankowskiorum Otto, 2019, paratype (WIRC), Burmese amber, Myanmar, body length: $6.5 \mathrm{~mm}$.

3.3.6. Tribe Selatosomini Schimmel, Tarnawski, Han and Platia, 2015 *

Selatosomini Schimmel, Tarnawski, Han and Platia, 2015: 30 [178]. Type genus: Selatosomus Stephens, 1830: 268 [179]. For more information, see Schimmel et al. [178].

\section{- Genus Selatosomus Stephens, 1830 *}

Selatosomus Stephens, 1830: 268 [179]. Type species: Elater aeneus Linnaeus, 1758: 406 [115]. For more information, see Cate [107].

\section{Selatosomus miegi Theobald, 1937}

Selatosomus miegi Theobald, 1937: 175 [42].

Type material. Holotype, sex unknown, elytron, compression fossil, R 624 (NHMB). 
Fossil deposit/age. Germany: Middle Member (Salt Formation), Kleinkembs; 33.928.4 Ma (Oligocene).

Literature. Theobald (1937: 175): original description [42].

Remark. The generic attribution of this species is unclear as this was described based only on elytral characters.

\subsubsection{Tribe Semiotini Jakobson, 1913 *}

Semiotina Jakobson, 1913: 736 [180]. Type genus: Semiotus Eschscholtz, 1829: 31 [117]. For more information, see Bouchard et al. [110].

- Genus Semiotus Eschscholtz 1829 *

Semiotus Eschscholtz 1829: 31 [117]. Type species: Elater furcatus Fabricius, 1775: 224 [181]. For more information, see Hyslop [111].

\section{Semiotus ehrenswaerdi (Heer, 1870)}

Elater ehrenswaerdi Heer, 1870: 74 [18].

Elater ehrenwaerdi: Scudder, 1891: 517 [24] [unavailable name, incorrect subsequent spelling not in prevailing usage; [129], Art. 33.3].

Elater ehrenwärdi [sic!]: Handlirsch, 1907: 745 [127].

Semiotus ehrensvaerdi: Birket-Smith, 1977: 18 [154] [unavailable name, incorrect subsequent spelling not in prevailing usage; [129], Art. 33.3].

Type material. Lectotype, sex unknown, elytron, compression fossil, No. 54a,b (SMNH). Paralectotype, sex unknown, elytron, compression fossil, No. 54c (SMNH).

Fossil deposit/age. Norway: Svalbard and Jan Mayen, Firkanten Formation, Cap Staratschin; 66.0-59.2 Ma (Paleocene).

Literature. Heer (1870: 74): original description [18]; Scudder (1891: 517): catalogue [24]; Handlirsch (1907: 745): catalogue [127]; Birket-Smith (1977: 18): taxonomic revision [154].

Remark. The description is based on a part of isolated elytron so the generic attribution of this species is rather problematic. However, based on the elytron reconstruction by Birket-Smith [154], it is really similar to that of Semiotus.

\section{Semiotus menatensis Piton, 1940}

Semiotus menatensis Piton, 1940: 179 [43].

Type material. Holotype, sex unknown, exoskeleton, compression fossil, No. 943 (MNHN).

Fossil deposit/age. France: Menat Formation, Menat; 61.6-59.2 Ma (Paleocene).

Literature. Piton (1940: 179): original description [43].

Remark. Based on the body proportions, shape of antenna and rather broad and short pronotum, this species strongly resembles recent Campsosternus (Oxynopterini). However, we prefer to postpone any taxonomic changes pending a comprehensive review including the type material.

\subsection{Subfamily Elaterinae Leach, 1815 *}

Elaterides Leach, 1815: 85 [114]. Type genus: Elater Linnaeus, 1758: 404 [115]. For more information including synonyms, see Bouchard et al. [110].

\subsubsection{Tribe Agriotini Laporte, 1840 *}

Agriotites Laporte, 1840: 233 [182]. Type genus: Agriotes Eschscholtz, 1829: 34 [117]. For more information, see Cate [107] and Bouchard et al. [110]. 


\section{- Genus Agriotes Eschscholtz, 1829*}

Agriotes Eschscholtz, 1829: 34 [117]. Type secies: Elater sputator Linnaeus, 1758: 405 [115]. For more information, see Cate [107].

\section{Agriotes comminutus Wickham, 1916}

Agriotes comminutus Wickham, 1916: 513 [28].

Type material. Holotype, sex unknown, exoskeleton, compression fossil, MCZ 2747 (= 11,800 in Scudder coll.) (MCZ).

Fossil deposit/age. USA: Colorado, Florissant Formation, Florissant; 37.2-33.9 Ma (Eocene).

Literature. Wickham (1916: 513): original description [28]; Wickham (1920: 354): catalogue [29].

\section{Agriotes nearcticus Wickham, 1916}

Agriotes nearcticus Wickham, 1916: 513 [28].

Type material. Holotype, sex unknown, exoskeleton, compression fossil, MCZ 2748 (=6653 in Scudder coll.) (MCZ).

Fossil deposit/age. USA: Colorado, Florissant Formation, Florissant; 37.2-33.9 Ma (Eocene).

Literature. Wickham (1916: 513): original description [28]; Wickham (1920: 354): catalogue [29].

\section{Agriotes succiniferusBecker, 1963}

Agriotes succiniferus Becker, 1963: 127 [46].

Agriotes succinifer: Zaragoza Caballero, 1990: 147 [61] [unavailable name, incorrect subsequent spelling not in prevailing usage; [129], Art. 33.3].

Type material. Holotype, female, exoskeleton, amber inclusion, No. 12,972 (UCMP).

Fossil deposit/age. Mexico: Simojovel region, Mexican (Chiapas) amber; 23.03-15.97 Ma (Miocene).

Literature. Becker (1963: 127): original description [46]; Spahr (1981: 46): catalogue [49]; Keilbach (1982: 247): catalogue [133]; Zaragoza Caballero (1990: 147): remark [61]; Schimmel (2005: 27): remark [91]; Solórzano Kraemer (2007: 119): checklist [90]; Schimmel and Tarnawski (2010: 363): remark [131]; Schimmel and Tarnawski (2012: 265): remark [132].

\subsubsection{Tribe Ampedini Gistel, 1848 *}

Ampedidae Gistel, 1848: 5 [138]. Type genus: Ampedus Dejean, 1833: 92 [139]. For more information, see Bouchard et al. [110].

\section{- Genus Ampedus Dejean, 1833 *}

Ampedus Dejean, 1833: 92 [139]. Type species: Elater sanguineus Linnaeus, 1758: 405 [115]. For more information see Sánchez-Ruiz [121] and Cate [107].

\section{Subgenus Octamenogonoides Iablokoff-Khnzorian, 1961}

Octamenogonoides Iablokoff-Khnzorian, 1961: 88 [47]. Type species: Elater (Octamenogonoides) gebleri Iablokoff-Khnzorian, 1961: 88 [47]. For more information, see Alekseev [92] and Kundrata et al. [12].

Remark. Iablokoff-Khnzorian [47] described Octamenogonoides as a subgenus in Elater Linnaeus, 1758, and Schimmel and Tarnawski [131] treated it is a member of the tribe Elaterini. Alekseev [92] transferred Octamenogonoides to Ampedus and kept its subgeneric status. 
Ampedus (Octamenogonoides) gebleri (Iablokoff-Khnzorian, 1961)

Elater (Octamenogonoides) gebleri Iablokoff-Khnzorian, 1961: 88 [47].

Elater gebleri: Larsson, 1978: 153 [48].

Ampedus (Octamenogonoides) gebleri: Alekseev, 2013: 7 [92].

Type material. Holotype, sex unknown, exoskeleton, amber inclusion, No. 364/641 (PIN).

Fossil deposit/age. Baltic amber; 38.0-33.9 Ma (Eocene).

Literature. Iablokoff-Khnzorian (1961: 88): original description [47]; Larsson (1978: 153): catalogue [48]; Spahr (1981: 48): catalogue [49]; Keilbach (1982: 246): catalogue [133]; Schimmel (2005: 27): remark [91]; Schimmel and Tarnawski (2010: 364): remark [131]; Alekseev (2013: 7): checklist [92]; Kundrata et al. (2020: 8): generic catalogue [12].

\section{Subgenus Ampedus Dejean, 1833}

Ampedus Dejean, 1833: 92 [139]. Type species: Elater sanguineus Linnaeus, 1758: 405 [115]. For more information see Sánchez-Ruiz [121] and Cate [107].

\section{Ampedus seyfriedii Heer, 1847}

Ampedus seyfriedii Heer, 1847: 131 [14].

Ampedus seyfriedi: Giebel, 1852: 651 [126] [unavailable name, incorrect subsequent spelling not in prevailing usage; [129], Art. 33.4].

Type material. Holotype, sex unknown, compression fossil (ETH).

Fossil deposit/age. Germany: Upper Freshwater-Molasse Formation, Öhningen, MN-7 mammal zone, Upper Öhningen beds Member; 12.7-11.608 Ma (Miocene).

Literature. Heer (1847: 131): original description [14]; Giebel (1852: 651): catalogue [126]; Giebel (1856: 97): redescription [16]; Heer (1861: 204): catalogue [171]; Heer (1865: 362): remark [17]; Heer (1872: 444): remark [135]; Heer (1876: 16): remark [136]; Handlirsch (1907: 743): catalogue [127].

\section{- Genus Ischnodes Germar, 1844 *}

Ischnodes Germar, 1844: 180 [183]. Type species: Elater sanguinicollis Panzer, 1793: 13 [184]. For more information, see Cate [107].

\section{Ischnodes gracilis Heer, 1847}

Ischnodes gracilis Heer, 1847: 133 [14].

Type material. Holotype, sex unknown, compression fossil, No. 7908 (ETH).

Fossil deposit/age. Germany: Upper Freshwater-Molasse Formation, Öhningen, Upper Öhningen beds; 12.7-11.608 Ma (Miocene).

Literature. Heer (1847: 133): original description [14]; Giebel (1852: 651): catalogue [126]; Giebel (1856: 97): redescription [16]; Scudder (1891: 542): catalogue [24]; Handlirsch (1907: 745): catalogue [127].

\subsubsection{Tribe Elaterini Leach, 1815 *}

Elaterides Leach, 1815: 85 [114]. Type genus: Elater Linnaeus, 1758: 404 [115]. For more information, including synonyms, see Bouchard et al. [110].

\section{- Genus Diaraphes Iablokoff-Khnzorian, 1961}

Diaraphes Iablokoff-Khnzorian, 1961: 89 [47]. Type species: Diaraphes kozhantshikovi Iablokoff-Khnzorian, 1961: 89 [47]. For more information, see Kundrata et al. [12].

Diaraphes kozhantshikovi Iablokoff-Khnzorian, 1961

Diaraphes kozhantshikovi Iablokoff-Khnzorian, 1961: 89 [47].

Type material. Holotype, sex unknown, exoskeleton, amber inclusion, No. 364/645 (PIN). 
Fossil deposit/age. Baltic amber; 38.0-33.9 Ma (Eocene).

Literature. Iablokoff-Khnzorian (1961: 89): original description [47]; Larsson (1978: 153): catalogue [48]; Spahr (1981: 47): catalogue [49]; Keilbach (1982: 246): catalogue [133]; Carpenter (1992: 304): generic catalogue [68]; Schimmel (2005: 27): remark [91]; Alekseev (2013: 7): checklist [92]; Kundrata et al. (2020: 8): generic catalogue [12].

\section{- Genus Elater Linnaeus, 1758 *}

Elater Linnaeus, 1758: 404 [115]. Type species: Elater ferrugineus Linnaeus, 1758: 405 [115]. For more information, see Cate [107].

Remark. Fossil species assigned to this genus urgently need a revision. It is possible that most, if not all, species in fact belong to another click-beetle genera. It should be also noted, that many older authors used the name "Elater" in erroneuous way (nec Elater Linnaeus [115]), i.e., for Ampedus (for more information, see Hyslop [111]), so they actually compared these fossil species with Ampedus and not with Elater.

\section{Elater asmodeus Zhang, 1989}

Elater asmodeus Zhang, 1989: 123 [58]. (SMJS).

Type material. Holotype, sex unknown, exoskeleton, compression fossil, No. s82747

Fossil deposit/age. China: Shanwang Formation, Shanwang, Linqu County; 20.4415.97 Ma (Miocene).

Literature. Zhang (1989: 123): original description [58]; Dong and Huang (2011: 1225): checklist [81].

Remark. This species superficially (i.e., in the body proportions and the structure of thorax) resembles Dendrometrinae rather than Elaterinae.

\section{Elater berryi Wickham, 1929}

Elater berryi Wickham, 1929: 148 [41].

Type material. Holotype, sex unknown, elytron, compression fossil, No. 80,474 (USNM)

Fossil deposit/age. USA: Tennessee, Cockfield Formation, 4 miles north of Jackson, Madison County; 41.3-38.0 Ma (Eocene).

Literature. Wickham (1929: 148): original description [41]; Wickham (1933: 103): catalogue [185].

\section{Elater burmitinus Cockerell, 1917}

Elater burmitinus Cockerell, 1917: 325 [33].

Type material. Holotype, sex unknown, exoskeleton, amber inclusion, No. 19,102 (BMNH).

Fossil deposit/age. Myanmar: Burmese amber; 99.6-93.5 Ma (Cretaceous).

Literature. Cockerell (1917: 325): original description [33]; Fletcher (1920: 987): remark [186]; Zherikhin (1978: 114): remark [187]; Spahr (1981: 47): catalogue [49]; Keilbach (1982: 247): checklist [133]; Poinar (1992: 144): remark [188]; Ross and York (2000: 12): catalogue [189]; Peris and Háva (2016: 496): remark [190].

Remark. Cockerell [33] suggested that it is not a member of true Elater; however, he was not able to assign it to any other genus. It was listed as "Elateridae sens. 1. burmitinus" by Keilbach [133]. The original description and available figure are not enough to make any conclusions about the placement of this species, and the proper study of the type specimen should be carried out in order to exclude the possibility that it is a member of Eucnemidae.

\section{Elater canabinus Zhang, 1989}

Elater canabinus Zhang, 1989: 124 [58]. (SMJS).

Type material. Holotype, sex unknown, exoskeleton, compression fossil, No. 840,105 
Fossil deposit/age. China: Shanwang Formation, Shanwang, Linqu County; 20.4415.97 Ma (Miocene).

Literature. Zhang (1989: 124): original description [58]; Dong and Huang (2011: 1225): checklist [81].

\section{Elater florissantensis Wickham, 1916}

Elater florissantensis Wickham, 1916: 510 [28].

Type material. Holotype, sex unknown, exoskeleton, compression fossil, MCZ 2752 (=8034 in Scudder coll.) (MCZ).

Fossil deposit/age. USA: Colorado, Florissant Formation, Florissant; 37.2-33.9 Ma (Eocene).

Literature. Wickham (1916: 510): original description [28]; Wickham (1920: 354): catalogue [29].

\section{Elater mitrus Zhang, 1989}

Elater mitrus Zhang, 1989: 122 [58].

Type material. Holotype, sex unknown, exoskeleton, compression fossil, No. s82713 (SMJS).

Fossil deposit/age. China: Shanwang Formation, Shanwang, Linqu County; 20.4415.97 Ma (Miocene).

Literature. Zhang (1989: 122): original description [58]; Dong and Huang (2011: 1225): checklist [81].

Remark. This species is similar to Ampedini in the body proportions, the narrowed campaniform pronotum, the elongate, almost parallel-sided elytra and the slightly serrated antenna. In Elaterini, the pronotum is usually wider, elytra not parallel sided, often somewhat wedge-shaped, and antenna more serrated. However, we prefer to keep this species tentatively in Elater until the holotype is examined in detail.

\section{Elater naumanni Giebel, 1856}

Elater naumanni Giebel, 1856: 91 [16]. tion).

Type material. Holotype, sex unknown, amber inclusion (Leipzig University collec-

Fossil deposit/age. Baltic amber; 38.0-33.9 Ma (Eocene).

Literature. Giebel (1856: 91): original description [16]; Handlirsch (1907: 744): catalogue [127]; Larsson (1978: 153): catalogue [48]; Keilbach (1982: 246): catalogue [133]; Alekseev (2013: 7): checklist [92].

Remark. This species was considered by Larsson [48] more similar to Limonius than to Elater. Type material should be studied in order to confirm the placement of this species.

\section{Elater rohweri Wickham, 1916}

Elater rohweri Wickham, 1916: 509 [28].

Type material. Holotype, sex unknown, compression fossil, No. 8227 (CUB).

Fossil deposit. USA: Colorado, Florissant Formation, Florissant, Station 14; 37.2-33.9 Ma (Eocene).

Literature. Wickham (1916: 509): original description [28]; Wickham (1920: 354): catalogue [29].

Remark. This species superficially resembles Ampedini in having slightly arcuate pronotum which is sinuate near posterior angles, and slightly elongated subparallel sided. However, we prefer to keep this species tentatively in Elater until the holotype is examined in detail.

Elater scudderi Wickham, 1916

Elater scudderi Wickham, 1916: 510 [28]. 
Type material. Holotype, sex unknown, exoskeleton, compression fossil, MCZ 2751 $(=12,485$ in Scudder coll.) (MCZ).

Fossil deposit. USA: Colorado, Florissant Formation, Florissant; 37.2-33.9 Ma (Eocene).

Literature. Wickham (1916: 510): original description [28]; Wickham (1920: 354): catalogue [29].

Remark. Wickham [28] already mentioned that this species probably does not belong to true Elater. Indeed, it has notably arcuate pronotum, with small posterior angles which is not a character usually found in Elaterinae. The generic attribution of E. scudderi needs further investigation.

\section{Elater wisniowskii Lomnicki, 1902}

Elater wisniowskii Lomnicki, 1902: 11 [25].

Type material. Holotype (probably), sex unknown, elytron, compression fossil (type depository unknown).

Fossil deposit/age. Ukraine: Bashkev Formation: Myszyn, Galicia; 13.65-12.7 Ma (Miocene).

Literature. Lomnicki (1902: 11): original description [25]; Handlirsch (1907: 745): catalogue [127].

Remark. The generic attribution of this species is unclear as it was described based only on elytral characters.

\section{- Genus Elatron Iablokoff-Khnzorian, 1961}

Elatron Iablokoff-Khnzorian, 1961: 90 [47]. Type species: Elatron semenovi IablokoffKhnzorian, 1961: 90 [47]. For more information, see Kundrata et al. [12].

\section{Elatron semenovi Iablokoff-Khnzorian, 1961}

Elatron semenovi Iablokoff-Khnzorian, 1961: 90 [47]. (PIN).

Type material. Holotype, sex unknown, exoskeleton, amber inclusion, No. 364/650

Fossil deposit/age. Baltic amber; 38.0-33.9 Ma (Eocene).

Literature. Iablokoff-Khnzorian (1961: 90): original description [47]; Larsson (1978: 153): catalogue [48]; Spahr (1981: 48): catalogue [49]; Keilbach (1982: 246): catalogue [133]; Carpenter (1992: 304): generic catalogue [68]; Schimmel (2005: 27): remark [91]; Alekseev (2013: 7): checklist [92]; Kundrata et al. (2020: 8): generic catalogue [12].

\section{- Genus Holopleurus Iablokoff-Khnzorian, 1961}

Holopleurus Iablokoff-Khnzorian, 1961: 86 [47]. Type species: Holopleurus succineus Iablokoff-Khnzorian, 1961: 86 [47]. For more information, see Kundrata et al. [12].

\section{Holopleurus succineus Iablokoff-Khnzorian, 1961}

Holopleurus succineus Iablokoff-Khnzorian, 1961: 86 [47].

Type material. Holotype, sex unknown, exoskeleton, amber inclusion, No. 364/530 (PIN).

Fossil deposit/age. Baltic amber; 38.0-33.9 Ma (Eocene).

Literature. Iablokoff-Khnzorian (1961: 86): original description [47]; Larsson (1978: 153): catalogue [48]; Spahr (1981: 48): catalogue [49]; Keilbach (1982: 246): catalogue [133]; Carpenter (1992: 304): generic catalogue [68]; Schimmel (2005: 27): remark [91]; Alekseev (2013: 7): checklist [92]; Kundrata et al. (2020: 8): generic catalogue [12].

\section{- Genus Orthoraphes Iablokoff-Khnzorian, 1961}

Orthoraphes Iablokoff-Khnzorian, 1961: 86 [47]. Type species: Orthoraphes reichardti Iablokoff-Khnzorian, 1961: 87 [47]. For more information, see Kundrata et al. [12]. 


\section{Orthoraphes reichardti Iablokoff-Khnzorian, 1961}

Orthoraphes reichardti Iablokoff-Khnzorian, 1961: 87 [47]. (PIN).

Type material. Holotype, sex unknown, exoskeleton, amber inclusion, No. 364/469

Fossil deposit/age. Baltic amber; 38.0-33.9 Ma (Eocene).

Literature. Iablokoff-Khnzorian (1961: 87): original description [47]; Larsson (1978: 153): catalogue [48]; Spahr (1981: 49): catalogue [49]; Keilbach (1982: 246): catalogue [133]; Carpenter (1992: 305): generic catalogue [68]; Schimmel (2005: 27): remark [91]; Alekseev (2013: 7): checklist [92]; Kundrata et al. (2020: 8): generic catalogue [12].

\subsubsection{Tribe Megapenthini Gurjeva, 1973 *}

Megapenthini Gurjeva, 1973: 448 [191]. Type genus: Megapenthes Kiesenwetter, 1858: 353 [192]. For more information, see Bouchard et al. [110].

\section{- Genus Abelater Fleutiaux, 1947 *}

Abelater Fleutiaux, 1947: 383 [193]. Type species: Melanoxanthus rubiginosus Candèze, 1878: 138 [194]. For more information, see Cate [107].

\section{Abelater succineus Schimmel, 2005}

Abelater succineus Schimmel, 2005: 106 [91].

Type material. Holotype, female, exoskeleton, amber inclusion, No. 4462 (CGG 2450) (GPIUH).

Fossil deposit/age. Baltic Amber; 38.0-33.9 Ma (Eocene).

Literature. Schimmel (2005: 106): original description [91]; Schimmel and Tarnawski (2010: 363): remark [131]; Schimmel and Tarnawski (2012: 265): remark [132]; Alekseev (2013: 7): checklist [92].

\section{- Genus Megapenthes Kiesenwetter, 1858 *}

Megapenthes Kiesenwetter, 1858: 353 [192]. Type species: Elater lugens Redtenbacher, 1842: 11 [195]. For more information, see Cate [107].

Megapenthes groehni Schimmel, 2005

Megapenthes groehni Schimmel, 2005: 107 [91].

Type material. Holotype, male, exoskeleton, amber inclusion, No. 4463 (CGG 1184) (GPIUH).

Fossil deposit/age. Baltic Amber; 38.0-33.9 Ma (Eocene).

Literature. Schimmel (2005: 107): original description [91]; Schimmel and Tarnawski (2010: 363): remark [131]; Schimmel and Tarnawski (2012: 265): remark [132]; Alekseev (2013: 7): checklist [92].

\section{Megapenthes primaevus Wickham, 1916}

Megapenthes primaevus Wickham, 1916: 511 [28].

Type material. Holotype, sex unknown, exoskeleton, compression fossil, MCZ 2750 $(=10,859$ in Scudder coll.) (MCZ).

Fossil deposit/age. USA: Colorado, Florissant Formation, Florissant; 37.2-33.9 Ma (Eocene).

Literature. Wickham (1916: 511): original description [28]; Wickham (1920: 354): catalogue [29]; Alekseev (2013: 7): checklist [92].

\section{Megapenthes voigti Schimmel, 2005}

Megapenthes voigti Schimmel, 2005: 110 [91].

Type material. Holotype, female, exoskeleton, amber inclusion, No. 4469 (CGG 4612) (GPIUH). Paratype, male, exoskeleton, amber inclusion, CGG 2389 (BMNH) (Figure 1B). 
Fossil deposit/age. Baltic Amber; 38.0-33.9 Ma (Eocene).

Literature. Schimmel (2005: 110): original description [91]; Schimmel and Tarnawski (2010: 363): remark [131]; Schimmel and Tarnawski (2012: 265): remark [132]; Alekseev (2013: 7): checklist [92].

\subsubsection{Tribe Physorhinini Candèze, 1859 *}

Physorhinites Candèze, 1859: 384 [148]. Type genus: Physorhinus Germar, 1840: 244 [196]. For more information, see Bouchard et al. [110].

\section{- Genus Anchastus LeConte, 185 *}

Anchastus LeConte, 1853: 459 [144]. Type species: Anchastus digitatus LeConte, 1853: 459 [144]. For more information, see Cate [107] and Johnson [106].

\section{Anchastus diluvialis Wickham, 1916}

Anchastus diluvialis Wickham, 1916: 507 [28].

Type material. Holotype, sex unknown, exoskeleton, compression fossil, MCZ 2759 $(=11,277$ in Scudder coll.) (MCZ).

Fossil deposit/age. USA: Colorado, Florissant Formation, Florissant; 37.2-33.9 Ma (Eocene).

Literature. Wickham (1916: 506): original description [28]; Wickham (1920: 354): catalogue [29].

\section{Anchastus eruptus Wickham, 1916}

Anchastus eruptus Wickham, 1916: 507 [28].

Type material. Holotype, sex unknown, exoskeleton, compression fossil, MCZ 2760 (=11,281 in Scudder coll.) (MCZ).

Fossil deposit/age. USA: Colorado, Florissant Formation, Florissant; 37.2-33.9 Ma (Eocene).

Literature. Wickham (1916: 506): original description [28]; Wickham (1920: 354): catalogue [29].

\subsubsection{Tribe Synaptini Gistel, 1856 *}

Synaptidae Gistel, 1856: 366 [170]. Type genus: Synaptus Eschscholtz, 1829: 32 [117]. For more information, see Cate [107] and Bouchard et al. [110].

\section{- Genus Glyphonyx Candèze, 1863 *}

Glyphonyx Candèze, 1863: 451 [145]. Type species: Glyphonyx gundlachii Candèze, 1863: 453 [145]. For more information, see Hyslop [111] and Cate [107].

\section{Glyphonyx chiapasensis Zaragoza Caballero, 1990}

Glyphonyx chiapasensis Zaragoza Caballero, 1990: 148 [61].

Type material. Holotype, probably male, amber inclusion (UNAM).

Fossil deposit/age. Mexico: Simojovel region, Mexican (Chiapas) amber; 23.03-15.97 Ma (Miocene).

Literature. Zaragoza Caballero (1990: 148): original description [61]; Solórzano Kraemer (2007: 119): catalogue [90].

Remark. The generic attribution of this species needs re-examination. Since this species has a very small body size and a rather unusual shape of body and prothorax (including a lack of the Synaptini-characteristic lateral basal indentations of pronotum), it might belong to another genus than Glyphonyx. 
Type material. Holotype, female, exoskeleton, amber inclusion, Nr. 12,873 (UCMP).

Fossil deposit/age. Mexico: Simojovel region, Mexican (Chiapas) amber; 23.0315.97 Ma (Miocene).

Literature. Becker (1963: 127): original description [46]; Spahr (1981: 48): catalogue [49]; Keilbach (1982: 247): catalogue [133]; Zaragoza Caballero (1990: 147): redescription [61]; Schimmel (2005: 27): remark [91]; Solórzano Kraemer (2007: 119): catalogue [90]; Schimmel and Tarnawski (2010: 363): remark [131]; Schimmel and Tarnawski (2012: 265): remark [132].

\subsubsection{Elaterinae Incertae Sedis}

\section{- Genus Crioraphes Iablokoff-Khnzorian, 1961}

Crioraphes Iablokoff-Khnzorian, 1961: 93 [47]. Type species: Crioraphes rohdendorfi Iablokoff-Khnzorian, 1961: 94 [47]. For more information, see Douglas [4] and Kundrata et al. [12].

Crioraphes rohdendorfi Iablokoff-Khnzorian, 1961

Crioraphes rohdendorfi Iablokoff-Khnzorian, 1961: 94 [47].

Crioraphes rhodendorfi: Larsson, 1978: 153 [48] [unavailable name, incorrect subsequent spelling not in prevailing usage; [129], Art. 33.3]. (PIN).

Type material. Holotype, sex unknown, exoskeleton, amber inclusion, No. 364/460

Fossil deposit/age. Baltic amber; 38.0-33.9 Ma (Eocene).

Literature. Iablokoff-Khnzorian (1961: 94): original description [47]; Larsson (1978: 153): catalogue [48]; Spahr (1981: 46): catalogue [49]; Keilbach (1982: 246): catalogue [133]; Carpenter (1992: 304): generic catalogue [68]; Alekseev (2013: 7): checklist [92]; Kundrata et al. (2020: 9): generic catalogue [12].

\subsection{Subfamily Lissominae Laporte, 1835 *}

Lissomidae Laporte, 1835: 178 [197]. Type genus: Lissomus Dalman, 1824: 13 [198]. For more information, including synonyms, see Kundrata et al. [12].

\subsubsection{Tribe Lissomini Laporte, 1835 *}

Lissomidae Laporte, 1835: 178 [197]. Type genus: Lissomus Dalman, 1824: 13 [198]. For more information, including synonyms, see Kundrata et al. [12].

\section{- Genus Lissomus Dalman, 1824 *}

Lissomus Dalman, 1824: 13 [198]. Type species: Lissomus punctulatus Dalman, 1824: 14 [198]. For more information, see Cate [107].

\section{Lissomus taxodii (Heer, 1870)}

Curculionites taxodii Heer, 1870: 76 [18].

Lissomus taxodii: Birket-Smith, 1977: 21 [154].

Type material. Holotype, sex unknown, elytron, compression fossil, No. 40 (SMNH).

Fossil deposit/age. Norway: Svalbard and Jan Mayen, Firkanten Formation, Cap Staratschin; 66.0-59.2 Ma (Paleocene).

Literature. Heer (1870: 76): original description [18]; Birket-Smith (1977: 21): revision, redescription [154]; Legalov (2015: 1497): catalogue (as Curculionites Heer, 1847) [199]; Legalov (2020: 23): catalogue (as Curculionites) [200].

Remark. The description of this species is based on a part of isolated elytron and, therefore, its generic attribution is rather problematic. It was originally described in Curculionites Heer, 1847 (Curculionoidea) and only later transferred to Lissomus in Elateridae [154]. Based on the reconstruction by Birket-Smith [154], it seems that this species belongs rather 
to Dendrometrinae than Lissominae. Legalov $[199,200]$ kept this species in original genus in Curculionoidea in the catalogues of fossil curculionoids. The identity of this species should be confirmed by study of the type material.

\subsubsection{Tribe Protelaterini Schwarz, 1902 *}

Protelateridae Schwarz, 1902: 365 [201]. Type genus: Protelater Sharp, 1877: 482 [202]. For more information, including synonyms, see Kundrata et al. [94,109].

\section{- Genus Baltelater Kundrata, Bukejs, Prosvirov and Hoffmannova, 2020}

Baltelater Kundrata, Bukejs, Prosvirov and Hoffmannova, 2020: 2 [94]. Type species: Baltelater bipectinatus Kundrata, Bukejs, Prosvirov and Hoffmannova, 2020: 3 [94]. For more information, see Kundrata et al. [94].

\section{Baltelater bipectinatus Kundrata, Bukejs, Prosvirov and Hoffmannova, 2020}

Baltelater bipectinatus Kundrata, Bukejs, Prosvirov and Hoffmannova, 2020: 3 [94].

Type material. Holotype, male, exoskeleton, amber inclusion, No. 6685 (JDC 8374)

(MAIG, ex coll. Jonas Damzen).

Fossil deposit/age. Baltic Amber; 38.0-33.9 Ma (Eocene).

Literature. Kundrata et al. (2020): original description [94].

3.6. Subfamily Negastriinae Nakane and Kishii, 1956 *

Negastriinae Nakane and Kishii, 1956: 203 [203]. Type genus: Negastrius Thomson, 1859: 106 [204]. For more information, see Bouchard et al. [110].

\section{- Genus Ganestrius Dolin, 1976}

Ganestrius Dolin, 1976: 69 [52]. Type species: Ganestrius stibicki Dolin, 1976: 71 [52]. For more information, see Kundrata et al. [12].

\section{Ganestrius elongatus Dolin, 1976}

Ganestrius elongatus Dolin, 1976: 71 [52].

Type material. Holotype, sex unknown, exoskeleton, compression fossil, No. 2066/2528 (part + counterpart) (PIN).

Fossil deposit/age. Kazakhstan: Karabastau Formation, Karatau, Mikhailovka; 166.1157.3 Ma (Jurassic).

Literature. Dolin (1976: 71): original description [52]; Dolin (1980: 77): revision, catalogue [53]; Korneev and Cate (2005: 15): checklist [120].

\section{Ganestrius stibicki Dolin, 1976}

Ganestrius stibicki Dolin, 1976: 71 [52].

Type material. Holotype, sex unknown, exoskeleton, compression fossil, No. 2066/2823 (PIN).

Fossil deposit/age. Kazakhstan: Karabastau Formation, Karatau, Mikhailovka; 166.1157.3 Ma (Jurassic).

Literature. Dolin (1976: 71): original description [52]; Dolin (1980: 77): revision, catalogue [53]; Carpenter (1992: 304): generic catalogue [68]; Korneev and Cate (2005: 10): checklist [120]; Kundrata et al. (2020: 9): generic catalogue [12].

\section{- Genus Paradonus Stibick, 1971 *}

Paradonus Stibick, 1971: 386 [205]. Type species: Elater pectoralis Say, 1839: 173 [206]. For more information, see Stibick [205]. 
Paradonus exterminatus (Wickham, 1916), comb. nov.

Cryptohypnus exterminatus Wickham, 1916: 506 [28].

Type material. Holotype, sex unknown, exoskeleton, compression fossil, MCZ 2762 (= 11,280 in Scudder coll.) (MCZ).

Fossil deposit/age. USA: Colorado, Florissant Formation, Florissant; 37.2-33.9 Ma (Eocene).

Literature. Wickham (1916: 506): original description [28]; Wickham (1920: 354): catalogue [29]; Stibick (1981: 246): systematic remark [164].

Remark. In his revision of Hypnoidinae, Stibick [164] examined Cryptohypnus exterminatus and suggested that it does not belong to Hypnoidinae. Instead, he suggested its placement in Negastriinae, close to Negastrius pectoralis group (=genus Paradonus sensu Stibick [205]), based on the "overall sculpture" and non-striate elytra. This relationship was already mentioned by Wickham [28] in the original description of C. exterminatus. Here, we formally place this species in Paradonus.

Paradonus hesperus (Wickham, 1916)

Cryptohypnus hesperus Wickham, 1916: 506 [28].

Paradonus hesperus: Stibick, 1971: 386 [205].

Type material. Holotype, sex unknown, exoskeleton, compression fossil, MCZ 2761 (=5294 in Scudder coll.) (MCZ).

Fossil deposit/age. USA: Colorado, Florissant Formation, Florissant; 37.2-33.9 Ma (Eocene).

Literature. Wickham (1916: 506): original description [28]; Wickham (1920: 354): catalogue [29]; Stibick (1971: 386): systematic remark [205]; Stibick (1981: 246): systematic remark [164].

\section{- Genus Protoquasimus Dolin, 1976}

Protoquasimus Dolin, 1976: 69 [52]. Type species: Protoquasimus brevicollis Dolin, 1976: 69 [52]. For more information, see Kundrata et al. [12].

\section{Protoquasimus brevicollis Dolin, 1976}

Protoquasimus brevicollis Dolin, 1976: 69 [52].

Type material. Holotype, sex unknown, exoskeleton, compression fossil, No. 2066/2993 (part + counterpart) (PIN).

Fossil deposit/age. Kazakhstan: Karabastau Formation, Karatau, Mikhailovka; 166.1157.3 Ma (Jurassic).

Literature. Dolin (1976: 69): original description [52]; Dolin (1980: 76): revision, catalogue [53]; Carpenter (1992: 305): generic catalogue [68]; Korneev and Cate (2005: 10): checklist [120]; Dong and Huang (2011: 1227): checklist [81]; Kundrata et al. (2020: 9): generic catalogue [12].

\subsection{Subfamily Omalisinae Lacordaire, 1857 *}

Homalisides Lacordaire, 1857: 303 [207]. Type genus: Omalisus Geoffroy, 1762: 179 [208]. For more information, see Bouchard et al. [110].

\section{- Genus Jantarokrama Kirejtshuk and Kovalev, 2015}

Jantarokrama Kirejtshuk and Kovalev, 2015: 1413 [93]. Type species: Jantarokrama utilis Kirejtshuk and Kovalev, 2015: 1414 [93]. For more information, see Kundrata et al. [12].

Jantarokrama utilis Kirejtshuk and Kovalev, 2015

Jantarokrama utilis Kirejtshuk and Kovalev, 2015: 1414 [93].

Type material. Holotype, male, amber inclusion, A52062 (283) (MNHN).

Fossil deposit/age. Baltic Amber; 38.0-33.9 Ma (Eocene). 
Literature. Kirejtshuk and Kovalev (2015: 1414): original description [93]; Kundrata et al. (2020: 9): generic catalogue [12]; Kundrata et al. (2020: 8): remark [94].

\subsection{Subfamily Pityobiinae Hyslop, 1917 *}

Pityobini Hyslop, 1917: 249 [209]. Type genus: Pityobius LeConte, 1853: 428 [144]. For more information, see Bouchard et al. [110].

\section{- Genus Cretopityobius Otto, 2019}

Cretopityobius Otto, 2019: 4 [96]. Type species: Cretopityobius pankowskiorum Otto, 2019: 4 [96]. For more information, see Otto [96] and Kundrata et al. [12]. The assignment of this genus to Pityobiinae needs further investigation.

\section{Cretopityobius pankowskiorum Otto, 2019}

Cretopityobius pankowskiorum Otto, 2019: 4 [96].

Type material. Holotype, sex unknown, amber inclusion (USNM). One paratype, sex unknown, amber inclusion (WIRC) (Figure 1C).

Fossil deposit/age. Myanmar: Burmese amber; 99.6-93.5 Ma (Cretaceous).

Literature. Otto (2019: 4): original description [96]; Kundrata et al. (2020: 9): generic catalogue [12]; Kundrata et al. (2020: 8): remark [94].

\subsection{Subfamily Protagrypninae Dolin, 1973}

Protagrypnini Dolin, 1973: 74 [50]. Type genus: Protagrypnus Dolin, 1973: 75 [50]. For more information, see Bouchard et al. [110] and Kundrata et al. [12].

Remark. This group was originally classified in Agrypninae [50,210]. Protagrypninae are mainly defined by the conspicuous structures on the prosternum and mesoventrite $[50,53,75,76]$. The validity of the first character was recently discussed and questioned by Muona et al. [99]. Protagrypninae are in urgent need of revision. Their monophyly, limits and systematic placement are unclear and it is possible that many lineages currently classified in this subfamily belong to another Elateridae group or even to other elateroid families, mainly Eucnemidae (see discussion in Muona et al. [99] and herein).

\subsubsection{Tribe Desmatini Dolin, 1975}

Desmatini Dolin, 1975: 60 [51]. Type genus: Desmatus Dolin, 1975: 60 [51]. For more information, see Bouchard et al. [110] and Kundrata et al. [12].

Remark. Representatives of this tribe differ from related groups mainly in the strongly developed metacoxal plates. This characters is typical for Elaterinae: Physorhinini but can be found in some other Elateridae and also in some other clicking elateroids such as Eucnemidae. Many species of this tribe indeed resemble Physorhinini in external characters, but not in all cases, so the composition and systematic position of Desmatini require further investigation (see discussion in Muona et al. [99]). Regarding genera formerly placed in Desmatini, Muona et al. [99] transferred Anoixis Chang, Kirejtshuk and Ren, 2010 (monotypic) to Eucnemidae: Palaeoxeninae, and Apoclion Chang, Kirejtshuk and Ren, 2010 (all 3 spp.) and Paradesmatus Chang, Kirejtshuk and Ren, 2009 (2 of 3 spp., including the type species) to Eucnemidae: Schizophilinae. One species of Paradesmatus was transferred to Desmatus Dolin, 1975.

\section{- Genus Desmatinus Chang, Kirejtshuk and Ren, 2010}

Desmatinus Chang, Kirejtshuk and Ren, 2010: 868 [86]. Type species: Desmatinus cognatus Chang, Kirejtshuk and Ren, 2010: 869 [86]. For more information, see Kundrata et al. [12].

Desmatinus cognatus Chang, Kirejtshuk et Ren, 2010

Desmatinus cognatus Chang, Kirejtshuk and Ren, 2010: 869 [86]. 
Type material. Holotype, sex unknown, exoskeleton, compression fossil, CNU-COLLB2008836 (CNU).

Fossil deposit. China: Liaoning Province, Shangyuan County, Beipiao City, Yixian Formation, Huangbanjigou, near Chaomidian Village; 125.45-122.46 Ma (Cretaceous).

Literature. Chang et al. (2010: 869): original description [86]; Dong and Huang (2011: 1225): checklist [81]; Yu et al. (2019: 383): remark [89]; Kundrata et al. (2020: 10): generic catalogue [12]; Muona et al. (2020: 9): revision [99].

\section{- Genus Desmatus Dolin, 1975}

Desmatus Dolin, 1975: 60 [51]. Type species: Desmatus lapidarius Dolin, 1975: 61 [51]. For more information, see Kundrata et al. [12].

Remark. This genus needs a revision since some species differ from the type species (and also from each other) in the body proportions, the shape of antenna, thorax, etc. The systematic placement of all species should be re-evaluated since they might represent Eucnemidae, based mainly on the presence of short and broad pronotum and enlarged metacoxal plates.

\section{Desmatus affinis Dolin, 1975}

Desmatus affinis Dolin, 1975: 62 [51].

Type material. Holotype, sex unknown, exoskeleton, compression fossil, No. 2554/672 (PIN).

Fossil deposit/age. Kazakhstan: Karabastau Formation, Karatau, Mikhailovka; 166.1157.3 Ma (Jurassic).

Literature. Dolin (1975: 62): original description [51]; Dolin (1980: 65): key, catalogue [53]; Korneev and Cate (2005: 11): checklist [120].

\section{Desmatus beckeri Dolin, 1975}

Desmatus beckeri Dolin, 1975: 62 [51].

Type material. Holotype, sex unknown, exoskeleton, compression fossil, No. 2066/2364 (part + counterpart) (PIN). Two paratypes, sex unknown, exoskeletons, compression fossils, Nos. 2554/689, 2384/489 (PIN).

Fossil deposit/age. Kazakhstan: Karabastau Formation, Karatau, Mikhailovka; 166.1157.3 Ma (Jurassic).

Literature. Dolin (1975: 62): original description [51]; Dolin (1980: 65): key, catalogue [53]; Korneev and Cate (2005: 12): checklist [120].

Desmatus lapidarius Dolin, 1975

Desmatus lapidarius Dolin, 1975: 61 [51].

Type material. Holotype, sex unknown, exoskeleton, compression fossil, No. 2066/3274 (PIN). Paratype, sex unknown, exoskeleton, compression fossil, No. 2784/1377 (PIN).

Fossil deposit/age. Kazakhstan: Karabastau Formation, Karatau, Mikhailovka; 166.1157.3 Ma (Jurassic).

Literature. Dolin (1975: 61): original description [51]; Dolin (1980: 64, 65): key, catalogue [53]; Carpenter (1992: 304): generic catalogue [68]; Korneev and Cate (2005: 10): checklist [120]; Kundrata et al. (2020: 10): generic catalogue [12].

Desmatus ponomarenkoi (Chang, Kirejtshuk and Ren, 2009)

Paradesmatus ponomarenkoi Chang, Kirejtshuk and Ren, 2009: 10 [78].

Desmatus ponomarenkoi: Muona et al., 2020: 10 [99].

Type material. Holotype, female, exoskeleton, impression, CNU-COL-NN2006876PC (CNU). Paratype, sex unknown, exoskeleton, impression, CNU-C-NN2007870 (CNU).

Fossil deposit/age. China: Inner Mongolia, Ningcheng County, Jiulongshan Formation, Daohugou; 166.1-157.3 Ma (Jurassic). 
Literature. Chang et al. (2009: 10): original description [78]; Chang et al. (2010: 867): remark [86]; Kirejtshuk et al. (2010: 791): checklist [87]; Dong and Huang (2011: 1225): checklist [81]; Schimmel and Tarnawski (2012: 265): remark [132]; Muona et al. (2020: 10): revision, nomenclatural change [99].

\section{Desmatus protensus Dolin, 1980}

Desmatus protensus Dolin, 1980: 65 [53]. (PIN).

Type material. Holotype, sex unknown, exoskeleton, compression fossil, No. 2997/2000

Fossil deposit/age. Kazakhstan: Karabastau Formation, Karatau, Mikhailovka; 166.1157.3 Ma (Jurassic).

Literature. Dolin (1980: 65): original description [53]; Korneev and Cate (2005: 22): checklist [120].

\section{- Genus Plesiorhaphes Dolin, 1980}

Plesiorhaphes Dolin, 1980: 65 [53]. Type species: Plesiorhaphes scaber Dolin, 1980: 66 [53]. For more information, see Kundrata et al. [12].

\section{Plesiorhaphes scaber Dolin, 1980}

Plesiorhaphes scaber Dolin, 1980: 66 [53].

Plesiorhaphes scabei: Carpenter, 1992: 305 [68].

Type material. Holotype, sex unknown, exoskeleton, compression fossil, No. 2784/1383 (PIN).

Fossil deposit/age. Kazakhstan: Karabastau Formation, Karatau, Mikhailovka; 166.1157.3 Ma (Jurassic).

Literature. Dolin (1980: 66): original description [53]; Carpenter (1992: 305): generic catalogue [68]; Korneev and Cate (2005: 10): checklist [120]; Kundrata et al. (2020: 10): generic catalogue [12].

\subsubsection{Tribe Hypnomorphini Dolin, 1975}

Hypnomorphini Dolin, 1975: 54 [51]. Type genus: Hypnomorphus Dolin, 1975: 54 [51]. For more information, see Bouchard et al. [110] and Kundrata et al. [12].

Remark. This tribe needs a revision as it most probably includes various unrelated groups.

\section{- $\quad$ Genus Abrotus Dolin, 1980}

Abrotus Dolin, 1980: 62 [53]. Type species: Abrotus sepultus Dolin, 1980: 63 [53]. The systematic placement of this genus is uncertain and should be re-examined. For more information, see Chang et al. [77] and Kundrata et al. [12].

\section{Abrotus reconditus Dolin, 1980}

Abrotus reconditus Dolin, 1980: 63 [53].

Type material. Holotype, sex unknown, exoskeleton, compression fossil, No. 2239/1451 (PIN). Paratype, sex unknown, exoskeleton, compression fossil, No. 2384/482 (PIN).

Fossil deposit/age. Kazakhstan: Karabastau Formation, Karatau, Mikhailovka; 166.1157.3 Ma (Jurassic).

Literature. Dolin (1980: 63): original description [53]; Korneev and Cate (2005: 22): checklist [120].

\section{Abrotus sepultus Dolin, 1980}

Abrotus sepultus Dolin, 1980: 63 [53].

Type material. Holotype, sex unknown, exoskeleton, compression fossil, No. 2066/3291 (PIN). Paratype, No. 2239/1418. 
Fossil deposit/age. Kazakhstan: Karabastau Formation, Karatau, Mikhailovka; 166.1157.3 Ma (Jurassic).

Literature. Dolin (1980: 63): original description [53]; Carpenter (1992: 304): generic catalogue [68]; Korneev and Cate (2005: 9): checklist [120]; Chang et al. (2011: 36): remark [77]; Kundrata et al. (2020: 11): generic catalogue [12].

\section{- Genus Adiagnostus Dolin, 1980}

Adiagnostus Dolin, 1980: 44 [53]. Type species: Adiagnostus cardiophorinus Dolin, 1980: 45 [53]. For more information, see Kundrata et al. [12].

\section{Adiagnostus ambiguus Dolin, 1980}

Adiagnostus ambiguus Dolin, 1980: 45 [53].

Type material. Holotype, sex unknown, exoskeleton, compression fossil, No. 2239/1423 (PIN).

Fossil deposit/age. Kazakhstan: Karabastau Formation, Karatau, Mikhailovka; 166.1157.3 Ma (Jurassic).

Literature. Dolin (1980: 45): original description [53]; Korneev and Cate (2005: 11): checklist [120].

\section{Adiagnostus cardiophorinus Dolin, 1980}

Adiagnostus cardiophorinus Dolin, 1980: 45 [53].

Type material. Holotype, sex unknown, exoskeleton, compression fossil, No. 2066/3231 (part) + 2066/3164 (counterpart) (PIN).

Fossil deposit/age. Kazakhstan: Karabastau Formation, Karatau, Mikhailovka; 166.1157.3 Ma (Jurassic).

Literature. Dolin (1980: 45): original description [53]; Carpenter (1992: 304): generic catalogue [68]; Korneev and Cate (2005: 9): checklist [120]; Kundrata et al. (2020: 11): generic catalogue [12].

\section{Adiagnostus minutulus Dolin, 1980}

Adiagnostus minutulus Dolin, 1980: 46 [53].

Type material. Holotype, sex unknown, exoskeleton, compression fossil, No. 2384/455 (PIN).

Fossil deposit/age. Kazakhstan: Karabastau Formation, Karatau, Mikhailovka; 166.1157.3 Ma (Jurassic).

Literature. Dolin (1980: 46): original description [53]; Korneev and Cate (2005: 20): checklist [120].

\section{- Genus Codemus Dolin, 1980}

Codemus Dolin, 1980: 35 [53]. Type species: Codemus synaptoides Dolin, 1980: 36 [53]. For more information, see Kundrata et al. [12].

\section{Codemus alatus Dolin, 1980}

Codemus alatus Dolin, 1980: 39 [53].

Type material. Holotype, sex unknown, exoskeleton, compression fossil, No. 2066/2734 (part) + 2066/2725 (counterpart) (PIN). Six paratypes, sex unknown, exoskeletons, compression fossils, Nos. 2554/654 (part + counterpart), 2784/1368, 2784/1391, 2904/901 (part + counterpart), 2904/926, $2997 / 4462$ (PIN).

Fossil deposit/age. Kazakhstan: Karabastau Formation, Karatau, Mikhailovka; 166.1157.3 Ma (Jurassic).

Literature. Dolin (1980: 39): original description [53]; Korneev and Cate (2005: 11): checklist [120]. 


\section{Codemus carinatus Dolin, 1980}

Codemus carinatus Dolin, 1980: 38 [53].

Type material. Holotype, sex unknown, exoskeleton, compression fossil, No. 2384/464 (PIN). Four paratypes, sex unknown, exoskeletons, compression fossils, Nos. 2384/483, 2784/1405, 2784/1372, 2384/1399 (PIN).

Fossil deposit/age. Kazakhstan: Karabastau Formation, Karatau, Mikhailovka; 166.1157.3 Ma (Jurassic).

Literature. Dolin (1980: 38): original description [53]; Korneev and Cate (2005: 13): checklist [120].

Remark. This species strongly differs from its congeners in the presence of long sublateral carinae on pronotum, the short incision of posterior edge of pronotum, and less elongated elytra. It is probable that $C$. carinatus belongs to another genus.

\section{Codemus jejunus Dolin, 1980}

Codemus jejunus Dolin, 1980: 39 [53].

Type material. Holotype, sex unknown, exoskeleton, compression fossil, No. 2066/3261 (PIN). Two paratypes, sex unknown, exoskeletons, compression fossils, Nos. 2239/1434, 2997/1960 (part) + 2997/1965 (counterpart) (PIN).

Fossil deposit/age. Kazakhstan: Karabastau Formation, Karatau, Mikhailovka; 166.1157.3 Ma (Jurassic).

Literature. Dolin (1980: 39): original description [53]; Korneev and Cate (2005: 17): checklist [120].

\section{Codemus martynovi Dolin, 1980}

Codemus martynovi Dolin, 1980: 37 [53].

Type material. Holotype, sex unknown, exoskeleton, compression fossil, No. 1784/37 (PIN).

Fossil deposit/age. Kazakhstan: Karabastau Formation, Karatau, Galkino; 166.1-157.3 Ma (Jurassic).

Literature. Dolin (1980: 37): original description [53]; Korneev and Cate (2005: 19): checklist [120].

\section{Codemus micros Dolin, 1980}

Codemus micros Dolin, 1980: 40 [53].

Type material. Holotype, sex unknown, exoskeleton, compression fossil, No. 2239/1441 (PIN). Six paratypes, sex unknown, exoskeletons, compression fossils, No. 2784/1384, 2904/917, 2904/924, 2904/925, 2997/4459, 2997/4460 (PIN).

Fossil deposit/age. Kazakhstan: Karabastau Formation, Karatau, Mikhailovka; 166.1157.3 Ma (Jurassic).

Literature. Dolin (1980: 40): original description [53]; Korneev and Cate (2005: 20): checklist [120].

\section{Codemus quadricolis Dolin, 1980}

Codemus quadricolis Dolin, 1980: 38 [53].

Codemus quadricollis Dolin, 1980: 35 [53] [also in the legend to Figure 28] [unavailable name, incorrect original spelling ([129], Art. 19.3); First Revisers ([129], Art. 24.2): Korneev and Cate (2005: 22) [120]].

Type material. Holotype, sex unknown, exoskeleton, compression fossil, No. 2066/2947 (part + counterpart) (PIN). Seven paratypes, sex unknown, exoskeletons, compression fossils, Nos. 2066/2628, 2066/2658, 2239/1437, 2384/500, 2384/502, 2554/687, 2554/704 (PIN).

Fossil deposit/age. Kazakhstan: Karabastau Formation, Karatau, Mikhailovka; 166.1157.3 Ma (Jurassic). 
Literature. Dolin (1980: 38): original description [53]; Korneev and Cate (2005: 22): checklist [120].

\section{Codemus sharovi Dolin, 1980}

Codemus sharovi Dolin, 1980: 36 [53].

Type material. Holotype, sex unknown, exoskeleton, compression fossil, No. 2384/474 (PIN). Five paratypes, sex unknown, exoskeletons, compression fossils, Nos. 2384/465, 2066/2722 (part + counterpart), 2784/1378, 2997/4471, 2997/417 (part + counterpart) (PIN).

Fossil deposit/age. Kazakhstan: Karabastau Formation, Karatau, Mikhailovka; 166.1157.3 Ma (Jurassic).

Literature. Dolin (1980: 36): original description [53]; Korneev and Cate (2005: 23): checklist [120].

\section{Codemus synaptoides Dolin, 1980}

Codemus synaptoides Dolin, 1980: 36 [53].

Type material. Holotype, sex unknown, exoskeleton, compression fossil, Nos. 2239/1411 (part) + 2239/1443 (counterpart) (PIN). 11 paratypes, sex unknown, exoskeletons, compression fossils, Nos. 2239/1433 (part + counterpart), 2066/2418, 2066/2696, 2066/3132, 2384/481 (part) + 2384/483 (counterpart), 2554/680, 2554/682, 2904/899 (part + counterpart), 2997/1994, 2997/2012, 2997/4473 (PIN).

Fossil deposit/age. Kazakhstan: Karabastau Formation, Karatau, Mikhailovka; 166.1157.3 Ma (Jurassic).

Literature. Dolin (1980: 36): original description [53]; Carpenter (1992: 304): generic catalogue [68]; Korneev and Cate (2005: 10): checklist [120]; Kundrata et al. (2020: 12): generic catalogue [12].

\section{Codemus teres Dolin, 1980}

Codemus teres Dolin, 1980: 38 [53].

Type material. Holotype, sex unknown, exoskeleton, compression fossil, No. 2904/905 (part + counterpart) (PIN). Five paratypes, sex unknown, exoskeletons, compression fossils, Nos. 2997/1996, 2997/2016, 2066/2703, 2066/2696, 2554/695 (PIN).

Fossil deposit/age. Kazakhstan: Karabastau Formation, Karatau, Mikhailovka; 166.1157.3 Ma (Jurassic).

Literature. Dolin (1980: 38): original description [53]; Korneev and Cate (2005: 24): checklist [120].

\section{Codemus zherichini Dolin, 1980}

Codemus zherichini Dolin, 1980: 37 [53].

Type material. Holotype, sex unknown, exoskeleton, compression fossil, No. 2997/1999 (PIN). Three paratypes, sex unknown, exoskeletons, compression fossils, Nos. 2997/4464, 2784/1367 (part + counterpart), 2784/1365 (part + counterpart) (PIN).

Fossil deposit/age. Kazakhstan: Karabastau Formation, Karatau, Mikhailovka; 166.1157.3 Ma (Jurassic).

Literature. Dolin (1980: 37): original description [53]; Korneev and Cate (2005: 25): checklist [120].

\section{- Genus Dolinelater Huber, Marggi and Menkveld-Gfeller, 2017}

Idiomorphus Dolin, 1980: 60 [53]. Type species: Idiomorphus singularis Dolin, 1980: 60 [53]. For more information, see Kundrata et al. [12]. Preoccupied by Idiomorphus Chaudoir, 1846 (Coleoptera: Carabidae) [211].

Dolinelater Huber, Marggi and Menkveld-Gfeller, 2017: 2 [211]. Replacement name for Idiomorphus Dolin, 1980. Erroneously omitted in the generic catalogue by Kundrata et al. [12]. 


\section{Dolinelater asperatus (Dolin, 1980)}

Idiomorphus asperatus Dolin, 1980: 61 [53].

Dolinelater asperatus: Huber et al. 2017: 2 [211].

Type material. Holotype, sex unknown, exoskeleton, compression fossil, No. 2066/3300 (PIN). Two paratypes, sex unknown, exoskeletons, compression fossils, Nos. 2997/2021, 2997/1967 (PIN).

Fossil deposit/age. Kazakhstan: Karabastau Formation, Karatau, Mikhailovka; 166.1157.3 Ma (Jurassic).

Literature. Dolin (1980: 61): original description [53]; Korneev and Cate (2005: 12): checklist [120]; Huber et al. (2017: 2): nomenclatural remark [211].

\section{Dolinelater singularis (Dolin, 1980)}

Idiomorphus singularis Dolin, 1980: 60 [53].

Dolinelater singularis: Huber et al., 2017: 2 [211].

Type material. Holotype, sex unknown, exoskeleton, compression fossil, No. 2239/1438 (PIN).

Fossil deposit/age. Kazakhstan: Karabastau Formation, Karatau, Mikhailovka; 166.1157.3 Ma (Jurassic).

Literature. Dolin (1980: 60): original description [53]; Carpenter (1992: 304): generic catalogue [68]; Korneev and Cate (2005: 10): checklist [120]; Huber et al. (2017: 2): nomenclatural remark [211]; Kundrata et al. (2020: 12): generic catalogue [12].

\section{- Genus Elaterophanes Handlirsch, 1906}

Elaterophanes Handlirsch, 1906: 436 [26]. Type species: Elater socius Giebel, 1856: 91 [16] (=Elater vetustus Brodie, 1845: 101 [13]). For more information, see Kundrata et al. [12].

Remark. The diagnosis, limits and systematic placement of this genus are unclear.

\section{Elaterophanes acutus Cockerell, 1916}

Elaterophanes acutus Cockerell, 1916: 478 [31].

Type material. Holotype, sex unknown, elytron, impression, No. 61,401 (USNM).

Fossil deposit/age. United Kingdom: Gloucestershire, Wainlode Cliff; 208.5-201.3 Ma (Triassic).

Literature. Cockerell (1916: 478): original description [31]; Handlirsch (1938: 65): catalogue [212].

Remark. The description of this species is based on an isolated elytron and, therefore, its generic attribution is rather problematic [212].

\section{Elaterophanes regius Whalley, 1985}

Elaterophanes regius Whalley, 1985: 165 [60].

Type material. Holotype, sex unknown, exoskeleton, compression fossil, No. 59,385 (BMNH). One paratype, No. 53,952 (BMNH).

Fossil deposit/age. United Kingdom: Charmouth Mudstone Formation, Dorset, Flatstones, Black Ven, Charmouth; 196.5-189.6 Ma (Jurassic).

Literature. Whalley (1985: 165): original description [60]; Martin (2010: 934): remark [73].

Elaterophanes vetustus (Brodie, 1845)

Elater vetustus Brodie, 1845: 101 [13].

Elater socius Giebel, 1856: 91 [16]. Synonymized with E. vetustus by Whalley (1985:

165) [60].

Elaterophanes socius: Handlirsch, 1906: 436 [26].

Elaterophanes vetustus: Handlirsch, 1906: 437 [26]. 
Type material. Elater vetustus. Holotype, sex unknown, compression fossil, No. NHM I.3576 (BMNH). Elater socius. Holotype, sex unknown, compression fossil, No. NHM I.3563 $(\mathrm{BMNH})$.

Fossil deposit/age. United Kingdom: Apperley, Lilstock Formation; 208.5-201.3 Ma.

Literature. Brodie (1845: 101): original description of E. vetustus [13]; Giebel (1856: 91): original description of E. vetustus, revision [16]; Phillips (1871: 123): checklist [213]; Heer (1883: 98): remark [137]; Scudder (1891: 204): catalogue [24]; Handlirsch (1906: 436): redescription of E. socius and E. vetustus [26]; Cockerell (1916: 478): catalogue [31]; Handlirsch (1938: 65, 69): checklist [212]; Haupt (1950: 102): remark [44]; Dolin (1973: 73): remark [50]; Whalley (1985: 165): taxonomy, remark [60]; Carpenter (1992: 304): generic catalogue [68]; Kundrata et al. (2020: 11): generic catalogue [12].

Remark. Brodie [13] described Elater vetustus based on a compression fossil from Lilstock Formation, Apperley (Triassic) in the United Kingdom. Heer [17] described Elaterites vetustus based on a single elytron from Schambelen, Aargau (Jurassic) in Switzerland, but he attributed the species name to Brodie. It is not clear whether Heer [17] thought that the fossil from Switzerland was conspecific with the Brodie's Elater vetustus from Apperley beds of the United Kingdom, and if/why he transferred that species from Elater to Elaterites. Later, Handlirsch [26] transferred Elater vetustus Brodie, 1845 to Elaterophanes (as he did with E. socius Giebel, 1856), and he erected a new genus, Dysarestus Handlirsch, 1906, for Elaterites vetustus Heer, 1865. He suggested that it might belong to Elaterophanes. Here, we follow Carpenter [68], who classified Dysarestus in Coleoptera incertae sedis.

\section{- $\quad$ Genus Graciolacon Dolin, 1980}

Graciolacon Dolin, 1980: 61 [53]. Type species: Graciolacon aeternus Dolin, 1980: 62 [53]. For more information, see Kundrata et al. [12].

Graciolacou: Dolin, 1980: legend of Figure 67 [53] [unavailable name, incorrect original spelling ([129], Art. 19.3); First Reviser ([129], Art. 24.2): Carpenter (1992: 304) [68]].

\section{Graciolacon aeternus Dolin, 1980}

Graciolacon aeternus Dolin, 1980: 62 [53].

Graciolacou [sic!] aethernus: Dolin, 1980: legend of drawing 67 [53].

Type material. Holotype, sex unknown, exoskeleton, compression fossil, No. 965/39 (PIN).

Fossil deposit/age. Kazakhstan: Karabastau Formation, Galkino, East Karatau; 166.1157.3 Ma (Jurassic).

Literature. Dolin (1980: 62): original description [53]; Carpenter (1992: 304): generic catalogue [68]; Korneev and Cate (2005: 11): checklist [120]; Kundrata et al. (2020: 11): generic catalogue [12].

\section{- Genus Hypnomorphoides Dolin, 1980}

Hypnomorphoides Dolin, 1980: 54 [53]. Type species: Hypnomorphoides catachtonius Dolin, 1980: 55 [53]. For more information, see Kundrata et al. [12].

Remark. This genus might belong to Eucnemidae as all its species have a compact body, with a short and broad thorax, and short elytra. Unfortunately, the main diagnostic characters [99] are either absent or not well visible on the original drawings or photographs [53].

\section{Hypnomorphoides angularis Dolin, 1980}

Hypnomorphoides angularis Dolin, 1980: 55 [53].

Type material. Holotype, sex unknown, exoskeleton, compression fossil, No. 2997/4461 (part + counterpart) (PIN).

Fossil deposit/age. Kazakhstan: Karabastau Formation, Karatau, Mikhailovka; 166.1157.3 Ma (Jurassic). 
Literature. Dolin (1980: 55): original description [53].

\section{Hypnomorphoides catachtonius Dolin, 1980}

Hypnomorphoides catachtonius Dolin, 1980: 55 [53].

Type material. Holotype, sex unknown, exoskeleton, compression fossil, No. 2066/3062 (PIN). Three paratypes, sex unknown, exoskeletons, compression fossils, Nos. 2904/916, 2997/2014, 2997/2028 (PIN).

Fossil deposit/age. Kazakhstan: Karabastau Formation, Karatau, Mikhailovka; 166.1157.3 Ma (Jurassic).

Literature. Dolin (1980: 55): original description [53]; Carpenter (1992: 304): generic catalogue [68]; Korneev and Cate (2005: 10): checklist [120]; Kundrata et al. (2020: 11): generic catalogue [12].

\section{Hypnomorphoides latus Dolin, 1980}

Hypnomorphoides latus Dolin, 1980: 56 [53].

Type material. Holotype, sex unknown, exoskeleton, compression fossil, No. 2239/1449 (PIN). Paratype, sex unknown, exoskeleton, compression fossil, No. 2384/449 (PIN).

Fossil deposit/age. Kazakhstan: Karabastau Formation, Karatau, Mikhailovka; 166.1157.3 Ma (Jurassic).

Literature. Dolin (1980: 56): original description [53]; Korneev and Cate (2005: 19): checklist [120].

\section{Hypnomorphoides procerulus Dolin, 1980}

Hypnomorphoides procerulus Dolin, 1980: 56 [53]. (PIN).

Type material. Holotype, sex unknown, exoskeleton, compression fossil, No. 2904/915

Fossil deposit/age. Kazakhstan: Karabastau Formation, Karatau, Mikhailovka; 166.1157.3 Ma (Jurassic).

Literature. Dolin (1980: 56): original description [53]; Korneev and Cate (2005: 22): checklist [120].

\section{- Genus Hypnomorphus Dolin, 1975}

Hypnomorphus Dolin, 1975: 54 [51]. Type species: Hypnomorphus rohdendorfi Dolin, 1975: 56 [51]. For more information, see Kundrata et al. [12].

Remark. This genus needs a revision since some species differ from the type species (and also from each other) in the body size, proportions, the shape of pronotum, elytra, etc. Most species probably belong to Eucnemidae based on the compact body, with a short and broad thorax, and short elytra. Additionally, $H$. rasnitzyni has antennae with last three antennomeres enlarged, which is a character present in Eucnemidae rather than Elateridae. Unfortunately, the main diagnostic characters [99] are usually absent or not well visible on the original drawings or photographs [51,53].

\section{Hypnomorphus aemulus Dolin, 1975}

Hypnomorphus aemulus Dolin, 1975: 56 [51].

Type material. Holotype, sex unknown, exoskeleton, compression fossil, No. 2554/692 (PIN). Three paratypes, sex unknown, exoskeletons, compression fossils, Nos. 2239/1417, 2239/1454, 2554/690 (PIN).

Fossil deposit/age. Kazakhstan: Karabastau Formation, Karatau, Mikhailovka; 166.1157.3 Ma (Jurassic).

Literature. Dolin (1975: 56): original description [51]; Dolin (1980: 28, 30): key, additional specimens: Nos. 2784/1385, 2784/1388 (PIN) [53]; Korneev and Cate (2005: 11): checklist [120]. 


\section{Hypnomorphus angulosus Dolin, 1980}

Hypnomorphus angulosus Dolin, 1980: 29 [53].

Type material. Holotype, sex unknown, exoskeleton, compression fossil, No. 2997/415 (part + counterpart) (PIN).

Fossil deposit/age. Kazakhstan: Karabastau Formation, Karatau, Mikhailovka; 166.1157.3 Ma (Jurassic).

Literature. Dolin (1980: 29): original description [53]; Korneev and Cate (2005: 11): checklist [120].

Hypnomorphus carpolithus Dolin, 1975

Hypnomorphus carpolithus Dolin, 1975: 57 [51]. (PIN).

Type material. Holotype, sex unknown, exoskeleton, compression fossil, No. 2066/2606

Fossil deposit/age. Kazakhstan: Karabastau Formation, Karatau, Mikhailovka; 166.1157.3 Ma (Jurassic).

Literature. Dolin (1975: 57): original description [51]; Dolin (1980: 27,30): key, additional specimens: Nos. 2997/419, 2997/2007, 2997/4457, 2784/1392, 2784/1386 (PIN) [53]; Korneev and Cate (2005: 13): checklist [120].

\section{Hypnomorphus confusus Dolin, 1975}

Hypnomorphus confusus Dolin, 1975: 59 [51]. (PIN).

Type material. Holotype, sex unknown, exoskeleton, compression fossil, No. 1789/213

Fossil deposit/age. Kazakhstan: Karabastau Formation, Karatau, Galkino; 166.1-157.3 Ma (Jurassic).

Literature. Dolin (1975: 59): original description [51]; Dolin (1980: 27): key, additional specimens: Nos. 2784/1382, 2784/1393, 2784/1401, 2904/903, 2904/910, 2997/1393, 2997/2009 (PIN) [53]; Korneev and Cate (2005: 14): checklist [120].

\section{Hypnomorphus curtus Dolin, 1980}

Hypnomorphus curtus Dolin, 1980: 28 [53].

Type material. Holotype, sex unknown, exoskeleton, compression fossil, No. 2066/2806 (PIN). Paratype, sex unknown, exoskeleton, compression fossil, No. 2384/472 (PIN).

Fossil deposit/age. Kazakhstan: Karabastau Formation, Karatau, Mikhailovka; 166.1157.3 Ma (Jurassic).

Literature. Dolin (1980: 28): original description [53]; Korneev and Cate (2005: 14): checklist [120].

\section{Hypnomorphus distinctus Dolin, 1975}

Hypnomorphus distinctus Dolin, 1975: 56 [51].

Type material. Holotype, sex unknown, exoskeleton, compression fossil, No. 2554/653 (part + counterpart) (PIN). Three paratypes, sex unknown, exoskeletons, compression fossils, Nos. 2554/652, 2239/1469, 2066/2764 (PIN).

Fossil deposit/age. Kazakhstan: Karabastau Formation, Karatau, Mikhailovka; 164.7155.7 Ma (Jurassic).

Literature. Dolin (1975: 56): original description [51]; Dolin (1980: 27,30): key, additional specimens: Nos. 2904/907 (part + counterpart), 2997/4470, 2904/923 (PIN) [53]; Korneev and Cate (2005: 14): checklist [120].

\section{Hypnomorphus dubius Dolin, 1975}

Hypnomorphus dubius Dolin, 1975: 60 [51]. (PIN).

Type material. Holotype, sex unknown, exoskeleton, compression fossil, No. 2554/651 
Fossil deposit/age. Kazakhstan: Karabastau Formation, Karatau, Mikhailovka; 166.1157.3 Ma (Jurassic).

Literature. Dolin (1975: 60): original description [51]; Dolin (1980: 27,30): key, additional specimens: Nos. 2239/1453, 2784/1403, 2997/428, 2997/1969 (PIN) [53]; Korneev and Cate (2005: 14): checklist [120].

\section{Hypnomorphus gigas Dolin, 1980}

Hypnomorphus gigas Dolin, 1980: 29 [53]. (PIN).

Type material. Holotype, sex unknown, exoskeleton, compression fossil, No. 2997/4472

Fossil deposit/age. Kazakhstan: Karabastau Formation, Karatau, Mikhailovka; 166.1157.3 Ma (Jurassic).

Literature. Dolin (1980: 29): original description [53]; Korneev and Cate (2005: 15): checklist [120].

\section{Hypnomorphus imperspicuus Dolin, 1975}

Hypnomorphus imperspicuus Dolin, 1975: 59 [51].

Type material. Holotype, sex unknown, exoskeleton, compression fossil, No. 2554/678 (PIN). Two paratypes, sex unknown, exoskeletons, compression fossils, Nos. 2066/3036, 2066/2838 (PIN).

Fossil deposit/age. Kazakhstan: Karabastau Formation, Karatau, Mikhailovka; 166.1157.3 Ma (Jurassic).

Literature. Dolin (1975: 59): original description [51]; Dolin (1980: 28,30): key, additional specimen: No. 2904/904 (PIN) [53]; Korneev and Cate (2005: 16): checklist [120].

\section{Hypnomorphus induratus Dolin, 1975}

Hypnomorphus induratus Dolin, 1975: 57 [51].

Type material. Holotype, sex unknown, exoskeleton, compression fossil, No. 2239/1427 (part + counterpart) (PIN).

Fossil deposit/age. Kazakhstan: Karabastau Formation, Karatau, Mikhailovka; 166.1157.3 Ma (Jurassic).

Literature. Dolin (1975: 57): original description [51]; Dolin (1980: 27,30): key, additional specimen: No. 2997/4468 (PIN) [53]; Korneev and Cate (2005: 10): checklist [120].

\section{Hypnomorphus inventus Dolin, 1975}

Hypnomorphus inventus Dolin, 1975: 57 [51].

Type material. Holotype, sex unknown, exoskeleton, compression fossil, No. 2384/491 (PIN). Four paratypes, sex unknown, exoskeletons, compression fossils, Nos. 2231/15 (Galkino), 2384/450, 2384/479, 2554/697 (Mikhailovka) (PIN).

Fossil deposit/age. Kazakhstan: Karabastau Formation, Karatau, Mikhailovka (type locality), Karatau, Galkino; 166.1-157.3 Ma (Jurassic).

Literature. Dolin (1975: 57): original description [51]; Dolin (1980: 27,30): key, additional specimens: Nos. 2384/498, 2784/1381, 2784/1364 (PIN) [53]; Korneev and Cate (2005: 17): checklist [120].

\section{Hypnomorphus minutus Dolin, 1975}

Hypnomorphus minutus Dolin, 1975: 59 [51].

Type material. Holotype, sex unknown, exoskeleton, compression fossil, No. 2239/1431 (PIN). 10 paratypes, sex unknown, exoskeletons, compression fossils, Nos. 2239/1428, 2239/1429, 2239/1430, 2239/1440 (part + counterpart), 2239/1424, 2554/691, 2554/655, 2554/700, 2066/2318, 2066/3248 (part) + 2066/2744 counterpart) (PIN).

Fossil deposit/age. Kazakhstan: Karabastau Formation, Karatau, Mikhailovka; 164.7155.7 Ma (Jurassic). 
Literature. Dolin (1975: 59): original description [51]; Dolin (1980: 27,30): key, additional specimens: Nos. 2784/1395, 2997/1981, 2997/2004, 2997/4457, 2997/4460 (PIN) [53]; Korneev and Cate (2005: 20): checklist [120].

\section{Hypnomorphus rasnitzyni Dolin, 1980}

Hypnomorphus rasnitzyni Dolin, 1980: 28 [53].

Type material. Holotype, male, exoskeleton, compression fossil, No. 2784/1362 (part + counterpart) (PIN). Paratype, female, sex unknown, exoskeleton, compression fossil, No. 2997/418 (PIN).

Fossil deposit/age. Kazakhstan: Karabastau Formation, Karatau, Mikhailovka; 164.7155.7 Ma (Jurassic).

Literature. Dolin (1980: 28): original description [53]; Korneev and Cate (2005: 22): checklist [120]; Dong et al. (2011: 482): remark [214].

Remark. Based on the habitus and shapes of antenna and pronotum, this species most probably belongs to Eucnemidae.

\section{Hypnomorphus rohdendorfi Dolin, 1975}

Hypnomorphus rohdendorfi Dolin, 1975: 56 [51].

Hypnomorphus rohdendorphi: Dolin, 1980: 28 [53] [unavailable name, incorrect subsequent spelling not in prevailing usage; [129], Art. 33.3].

Type material. Holotype, male, exoskeleton, compression fossil, No. 2384/457 (part + counterpart) (PIN). Three paratypes, sex unknown, exoskeletons, compression fossils, Nos. 2231/76 (Galkino), 2066/2341, 254/676 (Mikhailovka) (PIN).

Fossil deposit/age. Kazakhstan: Karabastau Formation, Karatau, Mikhailovka (type locality), Karatau, Galkino; 166.1-157.3 Ma (Jurassic).

Literature. Dolin (1975: 56): original description [51]; Dolin (1980: 28,30): key, additional specimens: Nos. 2997/415, 2997/416, 2997/4469 (PIN) [53]; Carpenter (1992: 304): generic catalogue [68]; Korneev and Cate (2005: 10): checklist [120]; Kundrata et al. (2020: 12): generic catalogue [12].

\section{- Genus Lapidiconides Dolin, 1980}

Lapidiconides Dolin, 1980: 43 [53]. Type species: Lapidiconides excellens Dolin, 1980: 43 [53]. For more information, see Kundrata et al. [12].

Remark. Species of this genus strongly resemble Eucnemidae in the compact body, with wide prothorax and relatively short elytra, and also Throscidae in almost trapezoidal pronotum. Unfortunately, the main diagnostic characters [99] are either absent or not well visible on the original drawings or photographs [53]. Additionally, L. innatus is most probably not congeneric with other two species.

\section{Lapidiconides brevis Dolin, 1980}

Lapidiconides brevis Dolin, 1980: 44 [53].

Type material. Holotype, sex unknown, exoskeleton, compression fossil, No. 2066/3156 (PIN).

Fossil deposit/age. Kazakhstan: Karabastau Formation, Karatau, Mikhailovka; 166.1157.3 Ma (Jurassic).

Literature. Dolin (1980: 44): original description [53]; Korneev and Cate (2005: 13): checklist [120].

\section{Lapidiconides excellens Dolin, 1980}

Lapidiconides excellens Dolin, 1980: 43 [53].

Lapidoconides [sic!] excellens Dolin, 1980: 43 [53].

Type material. Holotype, sex unknown, exoskeleton, compression fossil, No. 2066/2453 (PIN). 
Fossil deposit/age. Kazakhstan: Karabastau Formation, Karatau, Mikhailovka; 166.1157.3 Ma (Jurassic).

Literature. Dolin (1980: 43): original description [53]; Carpenter (1992: 304): generic catalogue [68]; Korneev and Cate (2005: 10): checklist [120]; Kundrata et al. (2020: 12): generic catalogue [12].

\section{Lapidiconides innatus Dolin, 1980}

Lapidiconides innatus Dolin, 1980: 44 [53].

Type material. Holotype, sex unknown, exoskeleton, compression fossil, No. 2784/1376 (part) + 2784/1400 (counterpart) (PIN).

Fossil deposit/age. Kazakhstan: Karabastau Formation, Karatau, Mikhailovka; 166.1157.3 Ma (Jurassic).

Literature. Dolin (1980: 44): original description [53]; Korneev and Cate (2005: 17): checklist [120].

Remark. This species differs considerably from its congeners in the large body size, shape of prosternum and hypomeron, less developed longitudinal sutures on prosternum, and more broadened metacoxal plates. Its systematic placement should be re-evaluated after the study of the type material.

\section{- $\quad$ Genus Lapidostenus Dolin, 1980}

Lapidostenus Dolin, 1980: 30 [53]. Type species: Lapidostenus infossus Dolin, 1980: 31 [53]. For more information, see Kundrata et al. [12].

Remark. This genus needs a revision since some species differ from the type species (and also from each other) in the body proportions, the shape of pronotum, elytra, etc. Most species probably belong to Eucnemidae based on the compact body, with a short and broad thorax, and short elytra. Only L. tarbinskyi Dolin, 1980 looks like a typical elaterid. Unfortunately, the main diagnostic characters [99] are usually absent or not well visible on the original drawings or photographs [53].

\section{Lapidostenus infossus Dolin, 1980}

Lapidostenus infossus Dolin, 1980: 31 [53].

Lapidostenus intossus: Dolin, 1980: legend of Figure 15 [53] [unavailable name, incorrect original spelling ([129], Art. 19.3); First Reviser ([129], Art. 24.2): Carpenter (1992: 304) [68]].

Type material. Holotype, sex unknown, exoskeleton, compression fossil, No. 2239/1439 (part + counterpart) (PIN). Two paratypes, sex unknown, exoskeletons, compression fossils, Nos. 2066/2431, 2997/2015 (part + counterpart) (PIN).

Fossil deposit. Kazakhstan: Karabastau Formation, Karatau, Mikhailovka; 166.1-157.3 Ma (Jurassic).

Literature. Dolin (1980: 31): original description [53]; Carpenter (1992: 304): generic catalogue [68]; Korneev and Cate (2005: 10): checklist [120]; Kundrata et al. (2020: 12): generic catalogue [12].

Lapidostenus insignis Dolin, 1980

Lapidostenus insignis Dolin, 1980: 32 [53].

Type material. Holotype, sex unknown, exoskeleton, compression fossil, No. 2784/1379 (PIN).

Fossil deposit. Kazakhstan: Karabastau Formation, Karatau, Mikhailovka; 166.1-157.3 Ma (Jurassic).

Literature. Dolin (1980: 32): original description [53]; Korneev and Cate (2005: 17): checklist [120].

Lapidostenus longicornis Dolin, 1980

Lapidostenus longicornis Dolin, 1980: 31/32 [53]. 
Lapidostenus lognicornis Dolin, 1980: 32 [53] [unavailable name, incorrect original spelling ([129], Art. 19.3); First Revisers ([129], Art. 24.2): Korneev and Cate (2005: 19) [120]]. (PIN).

Type material. Holotype, sex unknown, exoskeleton, compression fossil, No. 2784/1393

Fossil deposit/age. Kazakhstan: Karabastau Formation, Karatau, Mikhailovka; 166.1157.3 Ma (Jurassic).

Literature. Dolin (1980: 32): original description [53]; Korneev and Cate (2005: 19): checklist [120].

\section{Lapidostenus scutellaris Dolin, 1980}

Lapidostenus scutellaris Dolin, 1980: 31 [53].

Type material. Holotype, sex unknown, exoskeleton, compression fossil, No. 2066/2909 (PIN). Paratype, sex unknown, exoskeleton, compression fossil, No. 2997/2010 (PIN).

Fossil deposit/age. Kazakhstan: Karabastau Formation, Karatau, Mikhailovka; 166.1157.3 Ma (Jurassic).

Literature. Dolin (1980: 31): original description [53]; Korneev and Cate (2005: 23): checklist [120].

\section{Lapidostenus tarbinskyi Dolin, 1980}

Lapidostenus tarbinskyi Dolin, 1980: 32 [53]. (PIN).

Type material. Holotype, sex unknown, exoskeleton, compression fossil, No. 2239/1450

Fossil deposit/age. Kazakhstan: Karabastau Formation, Karatau, Mikhailovka; 166.1157.3 Ma (Jurassic).

Literature. Dolin (1980: 32): original description [53]; Korneev and Cate (2005: 24): checklist [120].

Remark. This species resembles Cardiophorinae in having the strongly arcuate sides of pronotum, with posterior angles short and curved inwards.

\section{- Genus Lithoptychus Dolin, 1980}

Lithoptychus Dolin, 1980: 57 [53]. Type species: Lithoptychus handlirschi Dolin, 1980: 57 [53]. For more information, see Kundrata et al. [12].

Remark: This genus might belong to Eucnemidae because its type species, L. handirschi, along with L. minutus Dolin, 1980, have a compact body, with a short and broad thorax, and short elytra. Unfortunately, the main diagnostic characters [99] are missing in the original figures [53], and, therefore, the taxonomic decision should be postponed until the type material is studied. Additionally, L. incertus Dolin, 1980 has antennae with last three antennomeres enlarged, which is a character present in Eucnemidae rather than Elateridae. Lithoptychus carinatissimus Dolin, 1980 externally also resembles Eucnemidae but it has very conspicuous sublateral carina on each side of pronotum.

\section{Lithoptychus carinatissimus Dolin, 1980}

Lithoptychus carinatissimus Dolin, 1980: 59 [53].

Type material. Holotype, sex unknown, exoskeleton, compression fossil, No. 2904/921 (PIN).

Fossil deposit/age. Kazakhstan: Karabastau Formation, Karatau, Mikhailovka; 166.1157.3 Ma (Jurassic).

Literature. Dolin (1980: 59): original description [53]; Korneev and Cate (2005: 13): checklist [120].

Lithoptychus handlirschi Dolin, 1980

Lithoptychus handlirschi Dolin, 1980: 57 [53]. 
Lithoptychus handlischi: Dolin, 1980: legend of Figure 60 [53] [unavailable name, incorrect original spelling ([129], Art. 19.3); First Reviser ([129], Art. 24.2): Carpenter (1992: 305) [68]].

Type material. Holotype, sex unknown, exoskeleton, compression fossil, No. 2554/696 (PIN). Paratype, sex unknown, exoskeleton, compression fossil, No. 2997/4439 (PIN).

Fossil deposit/age. Kazakhstan: Karabastau Formation, Karatau, Mikhailovka; 166.1157.3 Ma (Jurassic).

Literature. Dolin (1980: 57): original description [53]; Carpenter (1992: 305): generic catalogue [68]; Korneev and Cate (2005: 10): checklist [120]; Kundrata et al. (2020: 12): generic catalogue [12].

\section{Lithoptychus incertus Dolin, 1980}

Lithoptychus incertus Dolin, 1980: 58 [53].

Type material. Holotype, sex unknown, exoskeleton, compression fossil, No. 2452/41 (PIN). Three paratypes, sex unknown, exoskeletons, compression fossils, Nos. 2784/1373, 2784/1390, 2784/1398 (Mikhailovka) (PIN).

Fossil deposit/age. Kazakhstan: Karabastau Formation, Karatau, Galkino (type locality), Mikhailovka; 166.1-157.3 Ma (Jurassic).

Literature. Dolin (1980: 58): original description [53]; Korneev and Cate (2005: 16): checklist [120]; Dong and Huang (2011: 1228): morphological remark [81].

\section{Lithoptychus minutus Dolin, 1980}

Lithoptychus minutus Dolin, 1980: 58 [53].

Type material. Holotype, sex unknown, exoskeleton, compression fossil, No. 2239/1461 (PIN). Three paratypes, sex unknown, exoskeletons, compression fossils, Nos. 2239/1495, 2997/426, 2997/427 (PIN).

Fossil deposit/age. Kazakhstan: Karabastau Formation, Karatau, Mikhailovka; 166.1157.3 Ma (Jurassic).

Literature. Dolin (1980: 58): original description [53]; Korneev and Cate (2005: 20): checklist [120].

\section{- Genus Lithosomus Dolin, 1980}

Lithosomus Dolin, 1980: 46 [53]. Type species: Lithosomus erosus Dolin, 1980: 47 [53]. For more information, see Kundrata et al. [12].

Remark. The systematic placement of this genus needs to be re-evaluated since its type species might actually represent Eucnemidae.

\section{Lithosomus erosus Dolin, 1980}

Lithosomus erosus Dolin, 1980: 47 [53].

Type material. Holotype, sex unknown, exoskeleton, compression fossil, No. 2997/1987 (part) + 2997/1995 (counterpart) (PIN). Two paratypes, sex unknown, exoskeletons, compression fossils, Nos. 2997/1989, 2997/1967 (PIN).

Fossil deposit/age. Kazakhstan: Karabastau Formation, Karatau, Mikhailovka; 166.1157.3 Ma (Jurassic).

Literature. Dolin (1980: 47): original description [53]; Carpenter (1992: 305): generic catalogue [68]; Korneev and Cate (2005: 10): checklist [120]; Kundrata et al. (2020: 12): generic catalogue [12].

Remark. This species probably belongs to Eucnemidae due to its compact body, with a short and broad thorax, short elytra, and antennae with last three antennomeres enlarged. However, since the main diagnostic characters [99] are not well visible in the original figures [53], we prefer to postpone any taxonomic decision until the type material is examined in detail. 


\section{Lithosomus longicollis Dolin, 1980}

Lithosomus longicollis Dolin, 1980: 47 [53]. (PIN).

Type material. Holotype, sex unknown, exoskeleton, compression fossil, No. 2784/1363

Fossil deposit/age. Kazakhstan: Karabastau Formation, Karatau, Mikhailovka; 166.1157.3 Ma (Jurassic).

Literature. Dolin (1980: 47): original description [53]; Korneev and Cate (2005: 19): checklist [120].

Remark. Generic attribution of this species needs re-examination as it strongly differs from the type species of Lithosomus in the elongated pronotum, with slightly arcuate sides (pronotum short, broad, strongly campaniform in L. erosus), more elongated elytra (elytra rather short in L. erosus), more broadened metacoxal plates which are only slightly narrowed outwards (metacoxal plates notably narrowed outwards in L. erosus), and larger punctures in elytral striae (small punctures in L. erosus).

Although the body proportions of L. longicollis somewhat resemble Cardiophorinae or Negastriinae, with almost parallel-sided prosternal sutures and only slightly broadened prosternum being more typical for Cardiophorinae, there are no reliable characters that would point us to the proper systematic placement of this species.

\section{- Genus Necrocoelus Dolin, 1980}

Necrocoelus Dolin, 1980: 59 [53]. Type species: Necrocoelus aselloides Dolin, 1980: 59 [53]. For more information, see Kundrata et al. [12].

Remark. This genus might represent Cardiophorinae based on the globose pronotum, with short posterior angles, and a thickened and short prosternal process.

\section{Necrocoelus aselloides Dolin, 1980}

Necrocoelus aselloides Dolin, 1980: 59 [53].

Type material. Holotype, sex unknown, exoskeleton, compression fossil, No. 2066/2520 (part + counterpart) (PIN). Paratype, sex unknown, exoskeleton, compression fossil, No. 2904/909 (PIN).

Fossil deposit/age. Kazakhstan: Karabastau Formation, Karatau, Mikhailovka; 166.1157.3 Ma (Jurassic).

Literature. Dolin (1980: 59): original description [53]; Carpenter (1992: 305): generic catalogue [68]; Korneev and Cate (2005: 10): checklist [120]; Kundrata et al. (2020: 12): generic catalogue [12].

\section{- Genus Negastrioides Dolin, 1980}

Negastrioides Dolin, 1980: 52 [53]. Type species: Negastrioides tenuis Dolin, 1980: 52 [53]. For more information, see Kundrata et al. [12].

Remark. Species of this genus resemble Eucnemidae in having a broad prothorax with short pronotal posterior angles and a short prosternal process. Available figures in Dolin [53] also suggest that the type species, N. tenuis, along with N. globicollis Dolin, 1980, have a pedicel subapically attached to scape, a condition typical for Eucnemidae [99]. Basal antennomeres in remaining two species are absent in figures [53]. Systematic placement of Negastrioides needs further investigation.

Negastrioides globicollis Dolin, 1980

Negastrioides globicollis Dolin, 1980: 53 [53].

Type material. Holotype, sex unknown, exoskeleton, compression fossil, No. 2554/705 (PIN). Three paratypes, sex unknown, exoskeletons, compression fossils, Nos. 2784/1375, 2997/1972, 2997/1993 (PIN).

Fossil deposit/age. Kazakhstan: Karabastau Formation, Karatau, Mikhailovka; 166.1157.3 Ma (Jurassic). 
Literature. Dolin (1980: 53): original description [53]; Korneev and Cate (2005: 15): checklist [120].

Negastrioides tenuicornis Dolin, 1980

Negastrioides tenuicornis Dolin, 1980: 53 [53].

Type material. Holotype, sex unknown, exoskeleton, compression fossil, No. 2066/2847 (PIN). Three paratypes, sex unknown, exoskeletons, compression fossils, Nos. 2997/1971, 2997/2001, 2997/2006 (PIN).

Fossil deposit/age. Kazakhstan: Karabastau Formation, Karatau, Mikhailovka; 166.1157.3 Ma (Jurassic).

Literature. Dolin (1980: 53): original description [53]; Korneev and Cate (2005: 24): checklist [120].

Negastrioides tenuis Dolin, 1980

Negastrioides tenuis Dolin, 1980: 52 [53].

Type material. Holotype? male, exoskeleton, compression fossil, No. 2066/2320 (part + counterpart) (PIN). Three paratypes, sex unknown, exoskeletons, compression fossils, Nos. 2066/2886 (part + counterpart), 2239/1447, 2997/2002 (PIN).

Fossil deposit/age. Kazakhstan: Karabastau Formation, Karatau, Mikhailovka; 166.1157.3 Ma (Jurassic).

Literature. Dolin (1980: 52): original description [53]; Carpenter (1992: 305): generic catalogue [68]; Korneev and Cate (2005: 10): checklist [120]; Kundrata et al. (2020: 13): generic catalogue [12].

\section{Negastrioides tscherepanovi Dolin, 1980}

Negastrioides tscherepanovi Dolin, 1980: 54 [53].

Type material. Holotype, sex unknown, exoskeleton, compression fossil, No. 2066/2451 (PIN). Two paratypes, sex unknown, exoskeletons, compression fossils, Nos. 2997/424, 2997/4458 (part + counterpart) (PIN).

Fossil deposit/age. Kazakhstan: Karabastau Formation, Karatau, Mikhailovka; 166.1157.3 Ma (Jurassic).

Literature. Dolin (1980: 54): original description [53]; Korneev and Cate (2005: 24): checklist [120].

\section{- Genus Parahypnomorphus Dolin, 1980}

Parahypnomorphus Dolin, 1980: 33 [53]. Type species: Parahypnomorphus jurassicus Dolin, 1980: 33 [53]. For more information, see Kundrata et al. [12].

Remark. This genus might belong to Eucnemidae as all its species have a compact body, with a short and broad thorax, and short elytra. Illustrations of antennae also support this hypothesis [99], especially those of P. longicornis Dolin, 1980; however, they should be examined directly on the type material.

\section{Parahypnomorphus jurassicus Dolin, 1980}

Parahypnomorphus jurassicus Dolin, 1980: 33 [53].

Type material. Holotype, sex unknown, exoskeleton, compression fossil, No. 2554/703 (PIN).

Fossil deposit/age. Kazakhstan: Karabastau Formation, Karatau, Mikhailovka; 166.1157.3 Ma (Jurassic).

Literature. Dolin (1980: 33): original description [53]; Carpenter (1992: 305): generic catalogue [68]; Korneev and Cate (2005: 10): checklist [120]; Kundrata et al. (2020: 13): generic catalogue [12].

Parahypnomorphus longicornis Dolin, 1980

Parahypnomorphus longicornis Dolin, 1980: 34 [53]. 
Type material. Holotype, sex unknown, exoskeleton, compression fossil, No. 2239/1442 (PIN). Paratype, sex unknown, exoskeleton, compression fossil, No. 2997/1988 (PIN).

Fossil deposit. Kazakhstan: Karabastau Formation, Karatau, Mikhailovka; 1661.1157.3 Ma (Jurassic).

Literature. Dolin (1980: 34): original description [53]; Korneev and Cate (2005: 19): checklist [120].

\section{Parahypnomorphus similis Dolin, 1980}

Parahypnomorphus similis Dolin, 1980: 34 [53].

Type material. Holotype, sex unknown, exoskeleton, compression fossil, No. 2997/2013 (PIN).

Fossil deposit. Kazakhstan: Karabastau Formation, Karatau, Mikhailovka; 166.1-157.3 Ma (Jurassic).

Literature. Dolin (1980: 34): original description [53]; Korneev and Cate (2005: 23): checklist [120].

\section{- Genus Platyelater Dolin, 1980}

Platyelater Dolin, 1980: 40 [53]. Type species: Platyelater reflexicollis Dolin, 1980: 41 [53]. For more information, see Kundrata et al. [12].

Remark. All species other than the type species, P. reflexicollis, resemble Eucnemidae in having a compact body, with a short and broad thorax, and short elytra. Available figures of antennae also support this hypothesis. Additionally, P. figeratus Dolin, 1980 shares almost trapezoidal pronotum, with Throscidae. Systematic position of those species should be re-evaluated after study of the type material.

\section{Platyelater figeratus Dolin, 1980}

Platyelater figeratus Dolin, 1980: 42 [53].

Type material. Holotype, sex unknown, exoskeleton, compression fossil, No. 2239/1963 (PIN). Seven paratypes, sex unknown, exoskeletons, compression fossils, Nos. 2239/1408, 2239/1410 (female), 2239/1457, 2066/2461, 2904/920, 2997/1990, 2997/2003 (PIN).

Fossil deposit/age. Kazakhstan: Karabastau Formation, Karatau, Mikhailovka; 166.1157.3 Ma (Jurassic).

Literature. Dolin (1980: 42): original description [53]; Korneev and Cate (2005: 15): checklist [120].

Remark. This species notably differs from its congeners, especially in the body proportions and the shape of metacoxal plates.

\section{Platyelater quiescentus Dolin, 1980}

Platyelater quiescentus Dolin, 1980: 42 [53].

Type material. Holotype, sex unknown, exoskeleton, compression fossil, No. 2997/416 (PIN). Two paratypes, sex unknown, exoskeletons, compression fossils, Nos. 2784/1402, 2997/414 (part + counterpart) (PIN).

Fossil deposit/age. Kazakhstan: Karabastau Formation, Karatau, Mikhailovka; 166.1157.3 Ma (Jurassic).

Literature. Dolin (1980: 42): original description [53]; Korneev and Cate (2005: 22): checklist [120].

\section{Platyelater reflexicollis Dolin, 1980}

Platyelater reflexicollis Dolin, 1980: 41 [53].

Type material. Holotype, sex unknown, exoskeleton, compression fossil, No. 2904/900 (part + counterpart) (PIN). Six paratypes, sex unknown, exoskeletons, compression fossils, Nos. 2384/485, 2554/683, 2784/1387, 2904/906 (part + counterpart), 2904/914, 2997/423 (PIN). 
Fossil deposit/age. Kazakhstan: Karabastau Formation, Karatau, Mikhailovka; 166.1157.3 Ma (Jurassic).

Literature. Dolin (1980: 41): original description [53]; Carpenter (1992: 305): generic catalogue [68]; Korneev and Cate (2005: 10): checklist [120]; Dong et al. (2011: 482): remark [214]; Kundrata et al. (2020: 13): generic catalogue [12].

\section{Platyelater sukatschevae Dolin, 1980}

Platyelater sukatschevae Dolin, 1980: 41 [53]. (PIN).

Type material. Holotype, sex unknown, exoskeleton, compression fossil, No. 2997/2026

Fossil deposit/age. Kazakhstan: Karabastau Formation, Karatau, Mikhailovka; 166.1157.3 Ma (Jurassic).

Literature. Dolin (1980: 41): original description [53]; Korneev and Cate (2005: 24): checklist [120].

\subsubsection{Tribe Pollostelaterini Alekseev, 2011}

Pollostelaterini Alekseev, 2011: 424 [75]. Type genus: Pollostelater Alekseev, 2011: 424 [75]. For more information, see Kundrata et al. [12].

\section{- $\quad$ Genus Pollostelater Alekseev, 2011}

Pollostelater Alekseev, 2011: 424 [75]. Type species: Pollostelater baissensis Alekseev, 2011: 424 [75]. For more information, see Kundrata et al. [12].

Remark. Based on the original description and available images [75], we cannot exclude the possibility that this genus belongs to Eucnemidae due to the compact body, with a short and broad thorax. The longitudinal sutures on prosternum are obviously situated close to pronotosternal sutures, so they might be just deeply furrowed pronotosternal sutures as in some Eucnemidae. The presence of a sublateral carina on pronotum, which is a character typical for Eateridae rather than Eucnemidae, should be re-evaluated on the type material.

\section{Pollostelater baissensis Alekseev, 2011}

Pollostelater baissensis Alekseev, 2011: 424 [75].

Type material. Holotype, sex unknown, exoskeleton, impression, No. 3064/7100 (PIN). ceous).

Fossil deposit/age. Russia: Buryatia, Zaza Formation, Baissa; 125.0-113.0 Ma (Creta-

Literature. Alekseev (2011: 424): original description [75]; Kundrata et al. (2020: 13): generic catalogue [12].

\subsubsection{Tribe Protagrypnini Dolin, 1973}

Protagrypnini Dolin, 1973: 74 [50]. Type genus: Protagrypnus Dolin, 1973: 75 [50]. For more information, see Bouchard et al. [110] and Kundrata et al. [12].

\section{- Genus Acheonus Dolin, 1980}

Acheonus Dolin, 1980: 20 [53]. Type species: Acheonus abbreviatus Dolin, 1980: 21 [53]. For more information, see Kundrata et al. [12].

Remark. Species of this genus externally resemble Negastriinae but their placement in Elateridae should be rather confirmed by a study of type material. There are inconsistencies in original descriptions and corresponding images in Dolin [53] regarding the presence or absence of sublateral carinae in pronotal posterior angles. 
Type material. Holotype, sex unknown, exoskeleton, compression fossil, No. 2384/462 (PIN). Paratype, sex unknown, exoskeleton, compression fossil, No. 2384/462 [in the original description, holotype and paratype have the same number, which is probably an error] (PIN).

Fossil deposit/age. Kazakhstan: Karabastau Formation, Karatau, Mikhailovka; 166.1157.3 Ma (Jurassic).

Literature. Dolin (1980: 21): original description [53]; Carpenter (1992: 304): generic catalogue [68]; Korneev and Cate (2005: 9): checklist [120]; Schimmel and Tarnawski (2012: 265): remark [132]; Kundrata et al. (2020: 13): generic catalogue [12].

\section{Acheonus gracilis Dolin, 1980}

Acheonus gracilis Dolin, 1980: 21 [53]. (PIN).

Type material. Holotype, sex unknown, exoskeleton, compression fossil, No. 2784/1404

Fossil deposit/age. Kazakhstan: Karabastau Formation, Karatau, Mikhailovka; 166.1157.3 Ma (Jurassic).

Literature. Dolin (1980: 21): original description [53]; Schimmel and Tarnawski (2012: 265): remark [132].

Acheonus minutissimus Dolin, 1980

Acheonus minutissimus Dolin, 1980: 21 [53]. (PIN).

Type material. Holotype, sex unknown, exoskeleton, compression fossil, No. 2997/1992

Fossil deposit/age. Kazakhstan: Karabastau Formation, Karatau, Mikhailovka; 166.1157.3 Ma (Jurassic).

Literature. Dolin (1980: 21): original description [53]; Korneev and Cate (2005: 20): checklist [120]; Schimmel and Tarnawski (2012: 265): remark [132].

\section{- Genus Clavelater Dong and Huang, 2011}

Clavelater Dong and Huang, 2011: 1225 [81]. Type species: Clavelater ningchengensis Dong and Huang, 2011: 1226 [81]. For more information, see Kundrata et al. [12].

Remark. Muona et al. [99] suggested that this genus may represent an unknown lineage of Eucnemidae rather than Elateridae. They based their conclusions on the available literature and figures but the type material should be studied to solve the placement of Clavelater within Elateroidea. Nevertheless, we agree with the conclusions made by Muona et al. [99].

\section{Clavelater ningchengensis Dong and Huang, 2011}

Clavelater ningchengensis Dong and Huang, 2011: 1226 [81].

Type material. Holotype, sex unknown, exoskeleton, compression fossil, No. 151,836 (NIGP).

Fossil deposit/age. China: Inner Mongolia, Ningcheng County, Jiulongshan Formation, Daohugou; 166.1-157.3 Ma (Jurassic).

Literature. Dong and Huang (2011: 1226): original description [81]; Yu et al. (2019: 384): remark [89]; Kundrata et al. (2020: 14): generic catalogue [12]; Muona et al. (2020: 11): revision [99].

\section{- Genus Koreagrypnus Sohn and Nam, 2019}

Koreagrypnus Sohn and Nam in Sohn et al., 2019: 6 [76]. Type species: Koreagrypnus jinju Sohn and Nam in Sohn et al., 2019: 6 [76]. For more information, see Kundrata et al. [12]. 


\section{Koreagrypnus jinju Sohn and Nam, 2019}

Koreagrypnus jinju Sohn and Nam in Sohn et al., 2019: 6 [76].

Type material. Holotype, sex unknown, exoskeleton, compression fossil, GNUE-I2013002 and GNUE-I-2013002c (GNUE).

Fossil deposit/age. South Korea: Gyeongsangnamdo Province, Jinju Formation, Jeongchon Mountain, Jeongchon City, Jinju; 113.0-100.5 Ma (Cretaceous).

Literature. Sohn et al. (2019: 6): original description [76]; Kundrata et al. (2020: 14): generic catalogue [12].

\section{- Genus Lithocoelus Dolin, 1975}

Lithocoelus Dolin, 1975: 53 [51]. Type species: Lithocoelus detrusus Dolin, 1975: 53 [51]. For more information, see Kundrata et al. [12].

Remark. This genus might belong to Eucnemidae as both its species have a compact body, with a short and broad thorax, and relatively short elytra [51]. The type material needs to be checked for characters distinguishing (with more or less certainty) Elateridae from Eucnemidae [99].

\section{Lithocoelus detrusus Dolin, 1975}

Lithocoelus detrusus Dolin, 1975: 53 [51].

Type material. Holotype, sex unknown, exoskeleton, compression fossil, No. 2384/484 (PIN).

Fossil deposit/age. Kazakhstan: Karabastau Formation, Karatau, Mikhailovka; 166.1157.3 Ma (Jurassic).

Literature. Dolin (1975: 53): original description [51]; Dolin (1980: 20): remark [53]; Carpenter (1992: 305): generic catalogue [68]; Korneev and Cate (2005: 10): checklist [120]; Kundrata et al. (2020: 14): generic catalogue [12].

\section{Lithocoelus karatavicus Dolin, 1975}

Lithocoelus karatavicus Dolin, 1975: 54 [51].

Type material. Holotype, sex unknown, exoskeleton, compression fossil, No. 2239/1425 (part + counterpart) (PIN). Paratype, sex unknown, exoskeleton, compression fossil, No. 2335/82 (PIN).

Fossil deposit/age. Kazakhstan: Karabastau Formation, Karatau, Mikhailovka; 166.1157.3 Ma (Jurassic).

Literature. Dolin (1975: 54): original description [51]; Dolin (1980: 20): remark [53]; Korneev and Cate (2005: 18): checklist [120].

\section{- Genus Lithomerus Dolin, 1980}

Lithomerus Dolin, 1980: 23 [53]. Type species: Lithomerus cockerelli Dolin, 1980: 23 [53]. For more information, see Kundrata et al. [12].

Remark. This genus needs a revision since some species differ from the type species (and also from each other) in the body proportions, the shape of pronotum, elytra, etc. Lithomerus cockerelli (type species) and L. brevicollis Dolin, 1980 are probably correctly assigned to Elateridae but other species from the Palearctic deposits currently assigned to this genus might belong to Eucnemidae as they have a compact body, with a short and broad thorax, and short elytra. Lithomerus wunda Martin, 2010 from Australia is most probably a member of Throscidae [99].

\section{Lithomerus brachycollis Dolin, 1980}

Lithomerus brachycollis Dolin, 1980: 23 [53].

Type material. Holotype, sex unknown, exoskeleton, compression fossil, No. 2997/4463 (PIN). 
Fossil deposit/age. Kazakhstan: Karabastau Formation, Karatau, Mikhailovka; 166.1157.3 Ma (Jurassic).

Literature. Dolin (1980: 23): original description [53]; Martin (2010: 934): remark [73].

Remark. This species was mentioned by Dolin [53] only in the key on page 23 but the author obviously forgot to include the usual description which was otherwise available for all other new species in that paper. The holotype was figured in Figure 9 (drawing) and Plate I, Figure 3 (photograph), its collection number was mentioned in the figure legends, and the depository was specified in the introductory part of the paper [53]. The character states mentioned in the key on page 23 in Dolin [53] fulfill the requirements of Art. 13.1.1 [129] and, therefore, L. brachycollis is an available name.

\section{Lithomerus brevicollis Dolin, 1980}

Lithomerus brevicollis Dolin, 1980: 24 [53].

Type material. Holotype, sex unknown, exoskeleton, compression fossil, No. 2904/908 (PIN).

Fossil deposit/age. Kazakhstan: Karabastau Formation, Karatau, Mikhailovka; 166.1157.3 Ma (Jurassic).

Literature. Dolin (1980: 24): original description [53]; Dolin and Nel (2002: 341): remark [82]; Korneev and Cate (2005: 13): checklist [120]; Martin (2010: 934): remark [73]; Schimmel and Tarnawski (2012: 265): remark [132].

\section{Lithomerus buyssoni Dolin and Nel, 2002}

Lithomerus buyssoni Dolin and Nel, 2002: 341 [82].

Type material. Holotype, sex unknown, exoskeleton, compression fossil, MNHN-LPR.55231 (MNHN).

Fossil deposit/age. China: Liaoning Province, Yixian Formation, Beipiao City, Chaomidian Village; 125.45-122.46 Ma (Cretaceous).

Literature. Dolin and Nel (2002: 341): original description [82]; Kirejtshuk et al. (2010: 792): checklist [87]; Martin (2010: 934): remark [73]; Muona et al. (2020: 11): revision [99].

Remark. Muona et al. [99] suggested that this species might belong either to Elateridae: Protagrypninae or Eucnemidae: Palaeoxeninae but they did not study the holotype.

\section{Lithomerus cockerelli Dolin, 1980}

Lithomerus cockerelli Dolin, 1980: 23 [53].

Type material. Holotype, sex unknown, exoskeleton, compression fossil, No. 2384/454 (PIN). Seven paratypes, sex unknown, exoskeletons, compression fossils, Nos. 2384/460, 2384/492, 2784/1389, 2554/685, 2997/1977, 2997/1984, 2784/1366 (part + counterpart) (PIN).

Fossil deposit/age. Kazakhstan: Karabastau Formation, Karatau, Mikhailovka; 166.1157.3 Ma (Jurassic).

Literature. Dolin (1980: 23): original description [53]; Carpenter (1992: 305): generic catalogue [68]; Dolin and Nel (2002: 341): remark [82]; Korneev and Cate (2005: 10): checklist [120]; Martin (2010: 932): remark [73]; Schimmel and Tarnawski (2012: 265): remark [132]; Yu et al. (2019: 382): remark [89]; Kundrata et al. (2020: 14): generic catalogue [12].

\section{Lithomerus contiguus Dolin, 1980}

Lithomerus contiguus Dolin, 1980: 24 [53].

Lithomerus contiguous: Schimmel and Tarnawski, 2012: 265 [132] [unavailable name, incorrect subsequent spelling not in prevailing usage; [129], Art. 33.3].

Type material. Holotype, sex unknown, exoskeleton, compression fossil, No. 2066/2978 (PIN). Paratype, sex unknown, exoskeleton, compression fossil, No. 2997/2019 (PIN).

Fossil deposit/age. Kazakhstan: Karabastau Formation, Karatau, Mikhailovka; 166.1157.3 Ma (Jurassic). 
Literature. Dolin (1980: 24): original description [53]; Dolin and Nel (2002: 341): remark [82]; Korneev and Cate (2005: 14): checklist [120]; Martin (2010: 934): remark [73]; Schimmel and Tarnawski (2012: 265): remark [132].

\section{Lithomerus longulus Dolin, 1980}

Lithomerus longulus Dolin, 1980: 24 [53].

Lithomerus longulatus: Schimmel and Tarnawski, 2012: 265 [132] [unavailable name, incorrect subsequent spelling not in prevailing usage; [129], Art. 33.3]. (PIN).

Type material. Holotype, sex unknown, exoskeleton, compression fossil, No. 2066/3018

Fossil deposit/age. Kazakhstan: Karabastau Formation, Karatau, Mikhailovka; 166.1157.3 Ma (Jurassic).

Literature. Dolin (1980: 24): original description [53]; Dolin and Nel (2002: 341): remark [82]; Korneev and Cate (2005: 19): checklist [120]; Martin (2010: 934): remark [73]; Schimmel and Tarnawski (2012: 265): remark [132].

\section{Lithomerus wunda Martin, 2010}

Lithomerus wunda Martin, 2010: 932 [73].

Type material. Holotype, sex unknown, exoskeleton, compression fossil, WAM 08.179 (WAM). One paratype, sex unknown, exoskeleton, compression fossil, WAM 08.180 (WAM).

Fossil deposit/age. Australia: Cattamarra Coal Measures Formation, Mintaja M1 site; 182.7-174.1 Ma (Jurassic).

Literature. Martin (2010: 932): original description [73].

Remark. This species is obviously not conspecific with any other Lithomerus species. Based on the figures in the original description [73], Muona et al. [99] suggested that it should be placed within Throscidae based on the body size and the shape of antenna resting in the antennal groove. They postponed its transfer to Throscidae until the holotype is examined.

\section{- Genus Megalithomerus Sohn and Nam, 2019}

Megalithomerus Sohn and Nam in Sohn et al., 2019: 3 [76]. Type species: Megalithomerus magohalmii Sohn and Nam in Sohn et al., 2019: 3 [76]. For more information, see Kundrata et al. [12].

\section{Megalithomerus magohalmii Sohn and Nam, 2019}

Megalithomerus magohalmii Sohn and Nam in Sohn et al. 2019: 4 [76].

Type material. Holotype, sex unknown, exoskeleton, compression fossil, GNUE-I2013001 and GNUE-I-2013001c (GNUE).

Fossil deposit/age. South Korea: Gyeongsangnamdo Province, Jinju Formation, Jeongchon Mountain, Jeongchon City; 113.0-100.5 Ma (Cretaceous).

Literature. Sohn and Nam (2019: 4): original description [76]; Kundrata et al. (2020: 14): generic catalogue [12].

\section{- Genus Micragrypnites Dolin, 1973}

Micragrypnites Dolin, 1973: 76 [50]. Type species: Micragrypnites issykiensis Dolin, 1973: 77 [50]. For more information, see Kundrata et al. [12].

Remark. We cannot exclude the possibility that this genus might belong to Eucnemidae based on a short and broad prothorax with short posterior pronotal angles. Unfortunately, the main diagnostic characters [99] are either absent or not well visible on the images [53] and so the type material should be examined to confirm the placement of Micragrypnites in Elateridae. 


\section{Micragrypnites issykiensis Dolin, 1973}

Micragrypnites issykiensis Dolin, 1973: 77 [50].

Type material. Holotype, sex unknown, exoskeleton, impression, No. 371/1648 (PIN).

Fossil deposit/age. Kyrgyzstan: Dzhil Formation, Ak-Bulak-Say, Sogjuta, Issyk-Kul; 201.3-190.8 Ma (Jurassic).

Literature. Dolin (1973: 77): original description [50]; Carpenter (1992: 305): generic catalogue [68]; Korneev and Cate (2005: 10): check list [120]; Dong et al. (2011: 482): remark [214]; Kundrata et al. (2020: 14): generic catalogue [12].

\section{- Genus Paragrypnites Dolin, 1980}

Paragrypnites Dolin, 1980: 22 [53]. Type species: Paragrypnites jagemanni Dolin, 1980: 22 [53]. For more information, see Kundrata et al. [12].

\section{Paragrypnites jagemanni Dolin, 1980}

Paragrypnites jagemanni Dolin, 1980: 22 [53].

Type material. Holotype, sex unknown, exoskeleton, compression fossil, No. 2997/1980 (PIN).

Fossil deposit/age. Kazakhstan: Karabastau Formation, Karatau, Mikhailovka; 166.1157.3 Ma (Jurassic).

Literature. Dolin (1980: 22): original description [53]; Carpenter (1992: 305): generic catalogue [68]; Korneev and Cate (2005: 10): checklist [120]; Schimmel and Tarnawski (2012: 265): remark [132]; Kundrata et al. (2020: 15): generic catalogue [12].

\section{- Genus Paraprotagrypnus Chang, Zhao and Ren, 2009}

Paraprotagrypnus Chang, Zhao and Ren, 2009: 1433 [79]. Type species: Paraprotagrypnus superbus Chang, Zhao and Ren, 2009: 1434 [79]. For more information, see Kundrata et al. [12].

Remark. Muona et al. [99] examined this genus and concluded that it should be retained in Elateridae rather than transferred to Eucnemidae or Throscidae. They supported their conclusion by several morphological features, including antennomere II being attached apically to antennomere I. However, on the figures provided by Chang et al. [79], the antennomere II is attached somewhat subapically to antennomere I, and what is more, three apical antennomeres are enlarged, which is a character usually found in other clicking elateroids than Elateridae. The shape of prothorax, with broad and stout pronotum and anteriorly truncate prosternum, is also pointing to Eucnemidae. Another interesting character in this genus is a presence of sublateral carinae on pronotum, described and figured in Chang et al. [79]. These usually run from posterior angles more or less subparallel with sides. Muona et al. [99], however, wrote that "pronotal hind angles seemed to lack carina". Here, we follow Muona et al. [99], who studied the type material of the only species classified in Paraprotagrypnus, and retain this genus in Protagrypnini as originally proposed.

\section{Paraprotagrypnus superbus Chang, Zhao and Ren, 2009}

Paraprotagrypnus superbus Chang, Zhao and Ren, 2009: 1434 [79].

Type material. Holotype, sex unknown, exoskeleton, compression fossil, CNU-COLNN2006878 (CNU). One paratype, male, exoskeleton, compression fossil, CNU-COLNN2006879PC (CNU).

Fossil deposit/age. China: Inner Mongolia, Ningcheng County, Jiulongshan Formation, Shantou Township Daohugou; 166.1-157.3 Ma (Jurassic).

Literature. Chang et al. (2009: 1434): original description [79]; Kirejtshuk et al. (2010: 791): checklist [87]; Dong and Huang (2011: 1228): remark [81]; Yu et al. (2019: 382): remark [89]; Kundrata et al. (2020: 15): generic catalogue [12]; Muona et al. (2020: 10): revision [99]. 


\section{- Genus Protagrypnus Dolin, 1973}

Protagrypnus Dolin, 1973: 75 [50]. Type species: Protagrypnus exoletus Dolin, 1973: 75 [50]. For more information, see Kundrata et al. [12].

Remarks. In the original description of the genus, Dolin erroneously used the name Praelaterium Dolin, 1973 and vice versa, Protagrypnus was used in the original description of Praelaterium. However, species names were used correctly in both cases ([50], pp. 75, 78). Species in Protagrypnus do not seem to be congeneric. They differ considerably in body size, shape of pronotum and elytra, and shape and proportions of prosternum and hypomeron. We cannot exclude the possibility that the type species belongs to Eucnemidae.

\section{Protagrypnus exoletus Dolin, 1973}

Protagrypnus exoletus Dolin, 1973: 75 [50].

Type material. Holotype, sex unknown, exoskeleton, impression, No. 358/785 (PIN).

Fossil deposit/age. Kyrgyzstan: Dzhil Formation, Sogyuty, Issyk-Kul; 201.3-190.8 Ma (Jurassic).

Literature. Dolin (1973: 75): original description [50]; Carpenter (1992: 305): generic catalogue [68]; Korneev and Cate (2005: 10): checklist [120]; Dong and Huang (2009: 104): remark [80]; Chang et al. (2009: 10): remark [78]; Kundrata et al. (2020: 15): generic catalogue [12].

\section{Protagrypnus robustus Chang, Kirejtshuk and Ren, 2009}

Protagrypnus robustus Chang, Kirejtshuk and Ren, 2009: 11 [78].

Type material. Holotype, male, exoskeleton, impression, CNU-COL-NN2006843 (CNU). Two paratypes, exoskeletons, impressions, males, CNU-COL-NN2006875 and CNU-COL-NN2007869 (CNU).

Fossil deposit/age. China: Inner Mongolia, Ningcheng County, Jiulongshan Formation, Daohugou;166.1-157.3 Ma (Jurassic).

Literature. Chang et al. (2009: 11): original description [78]; Kirejtshuk et al. (2010: 791): checklist [87]; Dong and Huang (2011: 1227): remark [81]; Schimmel and Tarnawski (2012: 265): remark [132]; Yu et al. (2019: 381): remark [89]; Muona et al. (2020: 10): revision [99].

Remark. This species was examined by Muona et al. [99], who concluded that it should retain in Elateridae: Protagrypninae.

\section{- Genus Sinolithomerus Dong and Huang, 2009}

Sinolithomerus Dong and Huang, 2009: 103 [80]. Type species: Sinolithomerus dolini Dong and Huang, 2009: 104 [80]. For more information, see Kundrata et al. [12].

Remark. This genus might belong to Eucnemidae due to its relatively short and broad thorax, and short posterior angles of pronotum. Unfortunately, the main diagnostic characters [99] are either absent or not well visible on the original figures [53].

\section{Sinolithomerus dolini Dong and Huang, 2009}

Sinolithomerus dolini Dong and Huang, 2009: 104 [80]. (NIGP).

Type material. Holotype, sex unknown, exoskeleton, compression fossil, No. 149,367

Fossil deposit/age. China: Inner Mongolia, Ningcheng County, Haifanggou Formation, Beipiao City, Jiangjiagou village; 166.1-157.3 Ma (Jurassic).

Literature. Dong and Huang (2009: 104): original description [80]; Yu et al. (2019: 383): remark [89]; Kundrata et al. (2020: 15): generic catalogue [12]; Muona et al. (2020: 11): remark [99]. 


\subsection{Elateridae Incertae Sedis}

\section{- Genus Adocetus Scudder, 1900}

Adocetus Scudder, 1900: 97 [157]. Type species: Adocetus buprestoides Scudder, 1900: 97 [157]. For more information, see Kundrata et al. [12].

Remark. Based on the figure in original description, this genus might belong to Buprestidae rather than Elateridae. Therefore, the type material should be examined to confirm the proper family placement of Adocetus.

\section{Adocetus buprestoides Scudder, 1900}

Adocetus buprestoides Scudder, 1900: 97 [157].

Type material. Holotype, sex unknown, compression fossil (Newberry coll., Columbia University, NY, USA; based on the original description).

Fossil deposit/age. USA: Wyoming, Green River Formation, Bluffs by Twin Creek; 55.8-50.3 Ma (Eocene).

Literature. Scudder (1900: 97): original description [157]; Handlirsch (1907: 747): catalogue [127]; Carpenter (1992: 304): generic catalogue [68]; Kundrata et al. (2020: 16): generic catalogue [12].

\section{- Genus Artinama Lin, 1986}

Artinama Lin, 1986: 72 [55]. Type species: Artinama qinghuoensis Lin, 1986: 73 [55]. For more information, see Ponomarenko et al. [88] and Kundrata et al. [12].

Remark. Muona et al. [99] suggested that this genus belongs to Elateridae. Brief description of Artinama does not enable its placement even to a subfamily level. According to the images in Dong and Huang [81], Artinama resembles members of tribe Agrypnini (note that the drawing in that publication does not fully correspond with the photograph).

\section{Artinama qinghuoensis Lin, 1986}

Artinama qinghuoensis Lin, 1986: 73 [55].

Artinama qinghuanensis: Muona et al., 2020: 9 [99] [unavailable name, incorrect subsequent spelling not in prevailing usage; [129], Art. 33.3].

Type material. Holotype, sex unknown, exoskeleton, impression, No. 70,064 (NIGP).

Fossil deposit/age. China: Zaoshang Formation, Liuyang City, KHG 100, Shijiaba section, Wenjiashi; 199.3-190.8 Ma (Jurassic).

Literature. Lin (1986: 73): original description [55]; Dong et al. (2011: 483): remark [214]; Dong and Huang (2011: 1228): remark [81]; Ponomarenko et al. (2012: 480): revision [88]; Kundrata et al. (2020: 16): generic catalogue [12]; Muona et al. (2020: 9): revision [99].

\section{- Genus Bilineariselater Chang and Ren, 2008}

Bilineariselater Chang and Ren, 2008: 237 [84]. Type species: Bilineariselater foveatus Chang and Ren, 2008: 237 [84]. For more information, see Kundrata et al. [12].

Remark. This genus superficially resembles Selatosomini in having the frontal carina obsolete, the antenna weakly serrate, with elongated antennomere III, the pronotum rather broad, arcuate at sides and sinuate near hind angles, the posterior angles of pronotum moderately long, with sublateral carina, the prosternal lobe well developed, and elytra more or less ellipsoidal.

Bilineariselater foveatus Chang and Ren, 2008

Bilineariselater foveatus Chang and Ren, 2008: 237 [84].

Type material. Holotype, male, exoskeleton, compression fossil, CNU-C-LB2006801 $(\mathrm{CNU})$. 
Fossil deposit/age. China: Liaoning Province, Shangyan County, Beipiao City, Yixian Formation, Huangbanjigou, near Chaomidian Village; 125.45-122.46 Ma (Cretaceous).

Literature. Chang and Ren (2008: 237): original description [84]; Kirejtshuk et al. (2010: 792): checklist [87]; Dong and Huang (2011: 1225): checklist [81]; Yu et al. (2019: 382): remark [89]; Kundrata et al. (2020: 16): generic catalogue [12]; Muona et al. (2020: 9): revision [99].

\section{- Genus Cretoelaterium Alekseev, 2008}

Cretoelaterium Alekseev, 2008: 56 [74]. Type species: Cretoelaterium kazanovense Alekseev, 2008: 57 [74]. For more information, see Kundrata et al. [12].

Remark. We cannot exclude the possibility that this genus might belong to Eucnemidae.

Cretoelaterium kazanovense Alekseev, 2008

Cretoelaterium kazanovense Alekseev, 2008: 57 [74].

Type material. Holotype, sex unknown, exoskeleton, impression, No. 3693/1 (PIN).

Fossil deposit/age. Russia: Mirsanovo Formation, Chita Region, Shilka District, left bank of the Shilka river, Kazanovo railway station; 129.4-125.0 Ma (Cretaceous).

Literature. Alekseev (2008: 57): original description [74]; Kundrata et al. (2020: 16): generic catalogue [12].

\section{- Genus Cryptagriotes Wickham, 1916}

Cryptagriotes Wickham, 1916: 512 [28]. Type species: Cryptagriotes minusculus Wickham, 1916: 512 [28]. For more information, see Kundrata et al. [12].

Remark. Wickham [28] suggested that the body form of this genus is similar to Cryptohypnus Eschscholtz, 1830 (=Hypolithus Eschscholtz, 1829; Dendrometrinae: Hypnoidini) and the metacoxal plates are similar to those of Agriotes Eschscholtz, 1829 (Elaterinae: Agriotini). Based on the body size and proportions, and the more or less narrow prosternum with pronotosternal sutures almost parallel sided, this genus might belong to Cardiophorinae. However, its potential affinities to Eucnemidae should also be taken into account.

Cryptagriotes minusculus Wickham, 1916

Cryptagriotes minusculus Wickham, 1916: 512 [28].

Cryptagriotes minisculus: Carpenter, 1992: 304 [68] [unavailable name, incorrect subsequent spelling not in prevailing usage; [129], Art. 33.3].

Type material. Holotype, sex unknown, exoskeleton, compression fossil, MCZ 2749 (=8653 in Scudder coll.) (MCZ).

Fossil deposit/age. USA: Colorado, Florissant Formation, Florissant; 37.2-33.9 Ma (Eocene).

Literature. Wickham (1916: 512): original description [28]; Wickham (1920: 354): catalogue [29]; Hyslop (1921: 637): generic catalogue [111]; Carpenter (1992: 304): generic catalogue [68]; Kundrata et al. (2020: 7): catalogue [12].

\section{- Genus Cryptocoelus Dolin and Nel, 2002}

Cryptocoelus Dolin and Nel, 2002: 342 [82]. Type species: Cryptocoelus buffoni Dolin and Nel, 2002: 342 [82]. For more information, see Chang et al. [83] and Kundrata et al. [12].

Remark. Species in this genus superficially resemble Selatosomini in the frontal carina obsolete, the antenna more or less weakly serrate, the pronotum rather broad, arcuate at sides and sinuate near posterior angles, the posterior angles of pronotum moderately long, with sublateral carina, and elytra more or less ellipsoidal. 
Cryptocoelus baissensis Alekseev, 2011

Cryptocoelus baissensis Alekseev, 2011: 428 [75]. (PIN).

Type material. Holotype, sex unknown, exoskeleton, impression, No. 1989/2636

Fossil deposit/age. Russia: Buryatia, Zaza Formation, Baissa; 125.0-113.0 Ma (Cretaceous).

Literature. Alekseev (2011: 428): original description [75].

Cryptocoelus buffoni Dolin and Nel, 2002

Cryptocoelus buffoni Dolin and Nel, 2002: 342 [82].

Type material. Holotype, sex unknown, exoskeleton, compression fossil, MNHNLP-R.55227 (MNHN). Three paratypes, sex unknown, exoskeletons, compression fossils, MNHN-LP-R.55228, MNHN-LP-R.55229, MNHN-LP-R.55230 (MNHN).

Fossil deposit/age. China: Liaoning Province, Beipiao City, Yixian Formation, Chaomidian Village; 125.45-122.46 Ma (Cretaceous).

Literature. Dolin and Nel (2002: 342): original description [82]; Korneev and Cate (2005: 13): checklist [120]; Chang et al. (2007: 1248): remark [83]; Kirejtshuk et al. (2010: 792): checklist [87]; Alekseev (2011: 424): checklist [75]; Dong and Huang (2011: 1225): checklist [81]; Kundrata et al. (2020: 16): generic catalogue [12]; Muona et al. (2020: 9): revision [99].

Remark. Based on Figures 3-10 in Dolin and Nel [82], there are more species included in the type series of $C$. buffoni. The specimen in Figure 10 has a well developed prosternal lobe, while specimens in Figures 4, 6 and 8 have anterior portion of prosternum truncate. Additionally, the shape of elytra is also different among the figured specimens.

Cryptocoelus dolini Alekseev, 2011

Cryptocoelus dolini Alekseev, 2011: 430 [75].

Type material. Holotype, sex unknown, exoskeleton, impression, No. 4210/6420 (PIN). ceous).

Fossil deposit/age. Russia: Buryatia, Zaza Formation, Baissa; 125.0-113.0 Ma (Creta-

Literature. Alekseev (2011: 430): original description [75].

Cryptocoelus gianteus Chang, Ren and Shih, 2007

Cryptocoelus gianteus Chang, Ren and Shih, 2007: 1245 [83].

Cryptocoelus giganteus: Kirejtshuk et al., 2010: 792 [87] [unavailable name, incorrect subsequent spelling not in prevailing usage; [129], Art. 33.3].

Type material. Holotype, sex unknown, exoskeleton, compression fossil, CNU-CLB2006851-1, CNU-C-LB2006851-2 (CNU).

Fossil deposit/age. China: Liaoning Province, Shangyuan County, Beipiao City, Yixian Formation, Huangbanjigou, near Chaomidian Village; 125.45-122.46 Ma (Cretaceous).

Literature. Chang et al. (2007: 1245): original description [83]; Kirejtshuk et al. (2010: 792): checklist [87]; Alekseev (2011: 425): checklist [75]; Dong and Huang (2011: 1225): checklist [81]; Muona et al. (2020: 9): revision [99].

\section{Cryptocoelus lukashevichae Alekseev, 2011}

Cryptocoelus lukashevichae Alekseev, 2011: 428 [75].

Type material. Holotype, sex unknown, exoskeleton, impression, No. 3064/7102 (PIN). ceous).

Fossil deposit/age. Russia: Buryatia, Zaza Formation, Baissa; 125.0-113.0 Ma (Creta-

Literature. Alekseev (2011: 428): original description [75].

Cryptocoelus major Dolin and Nel, 2002 
Cryptocoelus major Dolin and Nel, 2002: 343 [82].

Crytocoleus [sic!] major: Yu et al., 2019: 381 [89].

Type material. Holotype, sex unknown, exoskeleton, compression fossil, MNHN-LPR.55226 (MNHN).

Fossil deposit/age. China: China: Liaoning Province, Shangyuan County, Beipiao City, Yixian Formation, Huangbanjigou, near Chaomidian Village; 125.45-122.46 Ma (Cretaceous).

Literature. Dolin and Nel (2002: 343): original description [82]; Korneev and Cate (2005: 10): checklist [120]; Chang et al. (2007: 1245): remark [83]; Chang et al. (2010: 873): remark [86]; Kirejtshuk et al. (2010: 787, 792): checklist [87]; Alekseev (2011: 424): checklist [75]; Dong and Huang (2011: 1225): checklist [81]; Yu et al. (2019: 381): remark [89]; Kundrata et al. (2020: 16): generic catalogue [12]; Muona et al. (2020: 9): revision [99].

\section{Cryptocoelus shcherbakovi Alekseev, 2011}

Cryptocoelus shcherbakovi Alekseev, 2011: 428 [75].

Type material. Holotype, sex unknown, exoskeleton, impression, No. 4210/6417 (PIN).

Fossil deposit/age. Russia: Buryatia, Zaza Formation, Baissa; 125.0-113.0 Ma (Cretaceous).

Literature. Alekseev (2011: 428): original description [75].

Cryptocoelus sinitshenkovae Alekseev, 2011

Cryptocoelus sinitshenkovae Alekseev, 2011: 427 [75].

Type material. Holotype, sex unknown, exoskeleton, No. 3002/2 (PIN).

Fossil deposit/age. Russia: Buryatia, Zaza Formation, Romanovka; 125.0-113.0 Ma (Cretaceous).

Literature. Alekseev (2011: 427): original description [75].

\section{- Genus Curtelater Chang and Ren, 2008}

Curtelater Chang and Ren, 2008: 238 [84]. Type species: Curtelater wui Chang and Ren, 2008: 239 [84]. For more information, see Kundrata et al. [12].

Remark. Muona et al. [99] examined this genus and confirmed that it belongs to Elateridae. However, based on the available morphological evidence, its position remains unclear, although it superficially resembles Selatosomini in several aspects including the morphology of head, antennae, and prothorax [84].

Curtelater wui Chang and Ren, 2008

Curtelater wui Chang and Ren, 2008: 239 [84]. (CNU).

Type material. Holotype, female, exoskeleton, compression fossil, CNU-C-LB2006830

Fossil deposit/age. China: Liaoning Province, Shangyuan County, Beipiao City, Yixian Formation, Huangbanjigou, near Chaomidian Village; 125.45-122.46 Ma (Cretaceous).

Literature. Chang and Ren (2008: 239): original description [84]; Kirejtshuk et al. (2010: 792): checklist [87]; Dong and Huang (2011: 1225): checklist [81]; Yu et al. (2019: 382): remark [89]; Kundrata et al. (2020: 17): generic catalogue [12]; Muona et al. (2020: 9): revision [99].

\section{- $\quad$ Genus Elateridium Tillyard, 1918}

Elateridium Tillyard, 1918: 751 [38]. Replacement name for Elaterites Tillyard, 1916: 41 [37]. Type species: Elaterites wianamattense Tillyard, 1916: 41 [37]. For more information, see Kundrata et al. [12].

Remark. The description of the type species of Elateridium is based on an isolated elytron, which makes the systematic placement of this genus rather problematic. Both 
species described by Dunstan [39] which are listed below should be excluded from Elateridae.

\section{Elateridium subulatum (Dunstan, 1923)}

Elaterites subulatus Dunstan, 1923: 44 [39].

Elateridium subulatum: Jell, 2004: 76 [215].

Type material. Holotype, sex unknown, elytron, compression fossil, Nr. 263a,b (part + counterpart) (QM; ex Geological Survey of Queensland).

Fossil deposit/age. Australia: Queensland, Blackstone Formation (Ipswich Coal Measures Group), Denmark Hill Insect Bed; 228.0-208.5 Ma (Triassic).

Literature. Dunstan (1923: 44): original description [39]; Handlirsch (1938: 13): catalogue [212]; Jell (2004: 76): catalogue [215]; Martins-Neto et al. (2006: 602): remark [71]; Martin (2010: 936): remark [73].

Remark. According to the drawing in the original description [39], it seems doubtful that this species belongs to Elateridae since the shape and structure of elytron are not typically elaterid like.

\section{Elateridium transversum (Dunstan, 1923)}

Elaterites trans versus Dunstan, 1923: 45 [39].

Elateridium transversum: Handlirsch, 1938: 13 [212].

Type material. Holotype, sex unknown, elytron, compression fossil, Nr. 159a, A (part+counterpart) (QM; ex Geological Survey of Queensland).

Fossil deposit/age. Australia: Queensland, Blackstone Formation (Ipswich Coal Measures Group), Denmark Hill Insect Bed; 228.0-208.5 Ma (Triassic).

Literature. Dunstan (1923: 45): original description [39]; Handlirsch (1938: 13): catalogue [212]; Jell (2004: 76): remark [215].

Remark. According to the drawing in the original description [39], it seems doubtful that this species belongs to Elateridae since the shape and structure of elytron are not typically elaterid like.

\section{Elateridium wianamattense (Tillyard, 1916)}

Elaterites wianamattensis Tillyard, 1916: 41 [37].

Elateridium wianamattense: Jell, 2004: 76 [215].

Type material. Holotype, sex unknown, elytron, impression, Nr. 130 (QM; ex Geological Survey of Queensland).

Fossil deposit. Australia: Ashfield Shales Formation, Carrington Brick Company's clay pit, St. Peter's; 247.2-242.0 Ma (Triassic).

Literature. Tillyard (1916: 41): original description [37]; Tillyard (1918: 751): nomenclatural remark [38]; Handlirsch (1938: 158): remark [212]; Carpenter (1992: 304): generic catalogue [68]; Jell (2004: 76): remark [215]; Kundrata et al. (2020: 17): generic catalogue [12].

\section{- $\quad$ Genus Elaterites Heer, 1847}

Elaterites Heer, 1847: 141 [14]. Type species: Elaterites lavateri Heer, 1847: 141 [14]. For more information, see Kundrata et al. [12].

Remark. This genus is obviously an assemblage of unrelated species of uncertain position. It includes taxa which authors were not possible to accommodate to any other genus in Elateridae [14,34]. Although it could be considered a "collective group" [129], some authors treated Elaterites as a genus with its own type species [127] and we follow this concept. The type species might represent Dendrometrinae based on the drawings in the original description [14]. 
Type material. Holotype, sex unknown, elytron, compression fossil (type depository unknown).

Fossil deposit/age. Switzerland: Greith coal mine, Hohenrhone; 28.4-23.03 Ma (Oligocene).

Literature. Heer (1847: 142): original description [14]; Giebel (1852: 651): catalogue [126]; Giebel (1856: 94): revision, redescription [16]; Scudder (1891: 518): catalogue [24]; Handlirsch (1907: 747): catalogue [127].

Remark. The description of E. amissus was based on an isolated elytron, which makes the generic attribution of this species rather problematic.

\section{Elaterites bruchi Cockerell, 1926}

Elaterites bruchi Cockerell, 1926: 320 [35].

Type material. Holotype, sex unknown, elytron, compression fossil (BMNH).

Fossil deposit/age. Argentina: Margas Verdes Formation, Station 2; Sunchal; 66.0-56.0 Ma (Paleocene).

Literature. Cockerell (1926: 320): original description [35]; Cockerell (1936: 1): checklist [216].

Remark. The description of E. bruchi was based on an isolated elytron, which makes the generic attribution of this species rather problematic.

\section{Elaterites dicrepidioides Deichmüller, 1881}

Elaterites dicrepidioides Deichmüller, 1881: 308 [21].

Type material. Unknown number of type specimens, probably only one, sex unknown, compression fossil (type depository unknown).

Fossil deposit/age. Czech Republic: Kučlín (u Bíliny); 37.2-33.9 Ma (Eocene).

Literature. Deichmüller (1881: 308): original description [21]; Scudder (1891: 518): catalogue [24]; Handlirsch (1907: 748): catalogue [127].

\section{Elaterites laconoides Cockerell, 1920}

Elaterites laconoides Cockerell, 1920: 457 [34].

Type material. Holotype, sex unknown, elytron, compression fossil, No. 18,998 (BMNH).

Fossil deposit/age. United Kingdom: Poole Formation, Bournemouth; 47.8-41.3 Ma (Eocene).

Literature. Cockerell (1920: 457): original description [34].

Remark. The description of E. laconoides was based on an isolated elytron, which makes the generic attribution of this species rather problematic.

\section{Elaterites lavateri Heer, 1847}

Elaterites lavateri Heer, 1847: 141 [14].

Type material. Holotype, sex unknown, compression fossil (ETH).

Fossil deposit/age. Germany: Upper Freshwater-Molasse Formation, Upper Öhningen beds, Öhningen; 12.7-11.608 Ma (Miocene).

Literature. Heer (1847: 141): original description [14]; Giebel (1852: 651): catalogue [126]; Giebel (1856: 94): revision, redescription [16]; Scudder (1891: 518): catalogue [24]; Handlirsch (1907: 747): catalogue [127]; Tillyard (1918: 751): remark [38]; Cockerell (1920: 457): remark [34]; Kundrata et al. (2020: 17): generic catalogue [12].

Remark. This species might represent Dendrometrinae based on the drawings in the original description [14]. It looks especially similar to genera such as Elathous Reitter, 1890, Limonius and Pheletes Kiesenwetter, 1858.

\section{Elaterites longus Haupt, 1956}

Elaterites longus Haupt, 1956: 48 [147].

Type material. Holotype, sex unknown, exoskeleton, compression fossil, G55/53 (GPIUH). 
Fossil deposit/age. Germany: Geiseltal; 47.8-41.3 Ma (Eocene).

Literature. Haupt (1956: 48): original description [147].

Remark. The description of E. longus was based only on the characters of elytra and abdomen, which makes the generic attribution of this species rather problematic.

\section{Elaterites microstictus Cockerell, 1926}

Elaterites microstictus Cockerell, 1926: 320 [35].

Type material. Holotype, sex unknown, elytron, compression fossil (part + counterpart) (BMNH, YPM).

Fossil deposit/age. Argentina: Margas Verdes Formation, Station 2; Sunchal; 66.0-56.0 Ma (Paleocene).

Literature. Cockerell (1926: 320): original description [35]; Cockerell (1936: 1): checklist [216].

Remark. The description of E. microstictus was based on an isolated elytron, which makes the generic attribution of this species rather problematic.

\section{Elaterites murchisoni (Giebel, 1856)}

Elaterium murchisoni Giebel, 1856: 93 [16].

Elaterites murchisoni: Cockerell, 1920: 456 [34].

Type material. Holotype, sex unknown, elytron, compression fossil, No. 18,996 (BMNH).

Fossil deposit/age. United Kingdom: Poole Formation, Dorset, Creech, between Corfe and Wareham; 47.8-41.3 Ma (Eocene).

Literature. Giebel (1856: 93): original description [16]; Scudder (1891: 518): catalogue [24]; Handlirsch (1907: 748): catalogue [127]; Cockerell (1920: 456): revision [34].

Remark. The description of E. murchisoni was based on an isolated elytron, which makes the generic attribution of this species rather problematic. It was treated as a member of Buprestidae by Westwood [15].

\section{Elaterites obsoletus Heer, 1847}

Elaterites obsoletus Heer, 1847: 142 [14].

Type material. Holotype, sex unknown, compression fossil, No. 7969 (ETH).

Fossil deposit/age. Germany: Upper Freshwater-Molasse Formation, Upper Öhningen; 12.7-11.608 Ma (Miocene).

Literature. Heer (1847: 142): original description [14]; Giebel (1852: 651): catalogue [126]; Giebel (1856: 94): revision, redescription [16]; Scudder (1891: 518): catalogue [24]; Handlirsch (1907: 747): catalogue [127].

\section{Elaterites palaeophilus Cockerell, 1920}

Elaterites palaeophilus Cockerell, 1920: 458 [34].

Type material. Holotype, sex unknown, elytron, compression fossil, No. 1467 (BMNH). (Eocene).

Fossil deposit/age. United Kingdom: Lambeth Group, Peckham; 56.0-47.8 Ma

Literature. Cockerell (1920: 458): original description [34].

Remark. The description of E. palaeophilus was based on an isolated elytron, which makes the generic attribution of this species rather problematic.

\section{Elaterites perditulus Cockerell, 1920}

Elaterites perditulus Cockerell, 1920: 457 [34].

Type material. Holotype, sex unknown, elytron, compression fossil, No. 10,418 (BMNH).

Fossil deposit/age. United Kingdom: Poole Formation, Dorset, Corfe, Isle of Purbeck; 47.8-41.3 Ma (Eocene).

Literature. Cockerell (1920: 457): original description [34]. 
Remark. The description of E. perditulus was based on an isolated elytron, which makes the generic attribution of this species rather problematic.

\section{Elaterites sculptilis Cockerell, 1920}

Elaterites sculptilis Cockerell, 1920: 458 [34].

Elater sculptilis: Birket-Smith, 1977: 20 [154].

Type material. Holotype, sex unknown, elytron, compression fossil, No. 10420, 10,422 (two impressions of the same specimen [34]) (BMNH).

Fossil deposit/age. United Kingdom: Poole Formation, Dorset, Corfe, Isle of Purbeck; 47.8-41.3 Ma (Eocene).

Literature. Cockerell (1920: 458): original description [34]; Birket-Smith (1977: 20): taxonomic remark [154].

Remark. Remark. The description of E. sculptilis was based on an isolated elytron, which makes the generic attribution of this species rather problematic. Birket-Smith [154] suggested that this species is presumably congeneric with Semiotus ehrenswaerdi but the size of both elytra differs significantly.

\section{- $\quad$ Genus Elaterium Westwood, 1854}

Elaterium Westwood, 1854: 387/393 [15]. Type species: Elaterium pronaeus Westwood, 1854: 387/393 [15]. For more information, see Kundrata et al. [12].

Remark. The description of the type species of Elaterium is based on a part of an isolated elytron, which makes the systematic placement of this genus rather problematic. A species described by Dunstan [39] is most probably not congeneric with E. pronaeus.

\section{Elaterium bipunctatum Dunstan, 1923}

Elaterium bipunctatum Dunstan, 1923: 47 [39].

Type material. Holotype, sex unknown, elytra, compression fossil, No. 292 (QM; ex Geological Survey of Queensland).

Fossil deposit/age. Australia: Blackstone Formation (Ipswich Coal Measures Group), Denmark Hill Insect Bed; 228.0-208.5 Ma (Triassic).

Literature. Dunstan (1923: 47): original description [39]; Handlirsch (1938: 14): catalogue [212]; Jell (2004: 76): remark [215].

Remark. According to the description and drawing in the original publication [39], elytron of this species bears strong costae which are usually not present in Elateridae. The placement of this species not only in Elaterium but even in the family Elateridae needs further examination.

\section{Elaterium pronaeus Westwood, 1854}

Elaterium pronaeus Westwood, 1854: 393 [15].

Type material. Holotype, sex unknown, compression fossil (BMNH).

Fossil deposit/age. United Kingdom: Lulworth Formation, Durlston Bay, Lower Purbeck, Swanage; 145.0-140.2 Ma (Cretaceous).

Literature. Westwood (1854: 393): original description [15]; Giebel (1856: 92): revision, redescription [16]; Scudder (1891: 205): catalogue [24]; Handlirsch (1906: 553): catalogue [26]; Cockerell (1920: 456): catalogue [34]; Coram and Jepson (2012: 60): catalogue [217]; Jell (2004: 76): remark [215]; Kundrata et al. (2020: 17): generic catalogue [12].

\section{- Genus Gripecolous Lin, 1986}

Gripecolous Lin, 1986: 80 [55]. Type species: Gripecolous enallus Lin, 1986: 80 [55]. For more information, see Kundrata et al. [12].

Remark. Muona et al. [99] suggested that this genus belongs either to Elateridae: Protagrypninae or to Eucnemidae. According to the images in Dong and Huang [81], Gripecolous resembles members of tribe Agrypnini, especially Agrypnus (note that drawing 
in that publication does not fully correspond with the photograph).

Gripecolous enallus Lin, 1986

Gripecolous enallus Lin, 1986: 80 [55].

Type material. Holotype, female (see Muona et al. [99]), exoskeleton, impression, No. 70,073 (NIGP).

Fossil deposit/age. China: Shiti Formation, KHG 201, Xiwan coal mine, Hezhou City; 170.3-168.3 Ma (Jurassic).

Literature. Lin (1986: 80): original description [55]; Dong et al. (2011: 482): revision [214]; Dong and Huang (2011: 1228): remark [81]; Ponomarenko et al. (2012: 482): revision [88]; Kundrata et al. (2020: 18): generic catalogue [12]; Muona et al. (2020: 11): revision [99].

\section{- Genus Ludiophanes Wickham, 1916}

Ludiophanes Wickham, 1916: 522 [28]. Type species: Ludiophanes haydeni Wickham, 1916: 522 [28]. For more information, see Kundrata et al. [12].

\section{Ludiophanes haydeni Wickham, 1916}

Ludiophanes haydeni Wickham, 1916: 522 [28].

Ludiophanes hayden: Carpenter, 1992: 305 [68] [unavailable name, incorrect subsequent spelling not in prevailing usage; [129], Art. 33.3].

Type material. Holotype, sex unknown, compression fossil, No. 90,386 (part + counterpart) (USNM).

Fossil deposit/age. USA: Colorado, Florissant Formation, Wilson Ranch, Florissant; 37.2-33.9 Ma (Eocene).

Literature. Wickham (1916: 522): original description [28]; Wickham (1920: 354): catalogue [29]; Carpenter (1992: 305): generic catalogue [68]; Kundrata et al. (2020: 18): generic catalogue [12].

\section{- $\quad$ Genus Mercata Lin, 1986}

Mercata Lin, 1986: 79 [55]. Type species: Mercata festira Lin, 1986: 79 [55]. For more information, see Kundrata et al. [12].

Remark. Chang et al. [77] placed Mercata in Cerophytidae but this was not followed by subsequent authors $[12,88,89,218]$. Ponomarenko et al. [88] placed this genus in Elateridae: Protagrypninae.

\section{Mercata festira Lin, 1986}

Mercata festira Lin, 1986: 79 [55].

Type material. Holotype, sex unknown, exoskeleton, impression, No. 70,072 (NIGP).

Fossil deposit/age. China: Shiti Formation, KHG 201, Xiwan coal mine, Hezhou City; 170.3-168.3 Ma (Jurassic).

Literature. Lin (1986: 79): original description [55]; Chang et al. (2011: 33): remark [77]; Dong et al. (2011: 486): revision [214]; Ponomarenko et al. (2012: 481): revision [88]; Kundrata et al. (2020: 18): generic catalogue [12]; Muona et al. (2020: 11): remark [99].

\section{- Genus Mionelater Becker, 1963}

Mionelater Becker, 1963: 125 [46]. Type species: Mionelater planatus Becker, 1963: 126 [46]. For more information, see Douglas [108] and Kundrata et al. [12].

Minonelater Schimmel, 2005: 27 [91] [unavailable name, incorrect subsequent spelling not in prevailing usage; [129], Art. 33.3].

Remark. Becker [46] placed this genus in Cardiophorinae. However, Douglas [108] suggested that this genus might belong to Dendrometrinae or another subfamily based on 
the serrate antennae, large eyes, shelf-like supra-antennal carina, elongate posterior angles of pronotum, and open mesocoxal cavities. Additionally, Mionelater has a pronotum more elongated than in typical Cardiophorinae, and almost not arcuate at sides, and according to the description [46], the prosternal process in this genus is long and rather narrow, while in Cardiophorinae, it is rather short and thick. The combination of characters, such as small body size, shape of supra-antennal carina, pronotum rather large compared to elytra, and elongate posterior angles of pronotum, suggests that Mionelater might be related to Hypnoidini. However, we keep it tentatively without a subfamilial assignment until further, more detailed, study is conducted.

\section{Mionelater planatus Becker, 1963}

Mionelater planatus Becker, 1963: 126 [46].

Type material. Holotype, probably male, exoskeleton, amber inclusion, No. 12,734 (UCMP).

Fossil deposit/age. Mexico: Simojovel region, Mexican (Chiapas) amber; 23.03-15.97 Ma (Miocene).

Literature. Becker (1963: 126): original description [46]; Spahr (1981: 49): catalogue [49]; Keilbach (1982: 247): catalogue [133]; Zaragoza Caballero (1990: 147): remark [61]; Carpenter (1992: 305): generic catalogue [68]; Schimmel (2005: 27): remark [91]; Solórzano Kraemer (2007: 119): catalogue [90]; Schimmel and Tarnawski (2010: 363): remark [131]; Schimmel and Tarnawski (2012: 265): remark [132]; Kundrata et al. (2020: 6): generic catalogue [12]; Kundrata et al. (2020: 8): remark [94].

\section{- $\quad$ Genus Ovivagina Zhang, 1997}

Ovivagina Zhang, 1997: 71 [56]. Type species: Ovivagina longa Zhang, 1997: 72 [56]. For more information, see Kundrata et al. [12].

Remark. The placement of this genus in Elateridae is uncertain and needs further research $[78,81,219]$.

Ovivagina longa Zhang, 1997

Ovivagina longa Zhang, 1997: 72 [56].

Type material. Holotype, sex unknown, exoskeleton, impression, 93-NA-3/K7,8 (?NIGP).

Fossil deposit/age. China: Badaowan Formation, Xinjiang, Shawan County, Nan'anchihai; 201.3-190.8 Ma (Jurassic).

Literature. Zhang (1997: 72): original description [56]; Yan and Zhang (2010: 451): remark [219]; Dong and Huang (2011: 1228): remark [81]; Kundrata et al. (2020: 18): generic catalogue [12]; Muona et al. (2020: 11): remark [99].

\section{- Genus Paralithomerus Chang, Zhang and Ren, 2008}

Paralithomerus Chang, Zhang and Ren, 2008: 55 [85]. Type species: Paralithomerus exquisitus Chang, Zhang and Ren, 2008: 55 [85]. For more information, see Kundrata et al. [12].

Remark. This genus was considered Protagrypninae incertae sedis [12] but Muona et al. [99] placed it to Elateridae incertae sedis.

Paralithomerus exquisitus Chang, Zhang and Ren, 2008

Paralithomerus exquisitus Chang, Zhang and Ren, 2008: 55 [85].

Paralithomerus exquisitius: Muona et al., 2020: 10 [99] [unavailable name, incorrect subsequent spelling not in prevailing usage; [129], Art. 33.3].

Type material. Holotype, sex unknown, exoskeleton, impression, CNU-C-LB20068741, CNU-C-LB2006874-2 (CNU). 
Fossil deposit/age. China: Liaoning Province, Shangyuan County, Beipiao City, Yixian Formation, Huangbanjigou, near Chaomidian Village; 125.45-122.46 Ma (Cretaceous).

Literature. Chang et al. (2008: 55): original description [85]; Kirejtshuk et al. (2010: 792): checklist [87]; Dong and Huang (2011: 1225): checklist [81]; Yu et al. (2019: 382): remark [89]; Kundrata et al. (2020: 15): generic catalogue [12]; Muona et al. (2020: 10): revision [99].

\section{Paralithomerus parallelus Chang, Zhang and Ren, 2008}

Paralithomerus parallelus Chang, Zhang and Ren, 2008: 58 [85]. (CNU).

Type material. Holotype, sex unknown, exoskeleton, impression, CNU-C-LB2006872

Fossil deposit. China: Liaoning Province, Shangyuan County, Beipiao City, Yixian Formation, Huangbanjigou, near Chaomidian Village; 125.45-122.46 Ma (Cretaceous).

Literature. Chang et al. (2008: 58): original description [85]; Kirejtshuk et al. (2010: 792): checklist [87]; Dong and Huang (2011: 1225): checklist [81]; Muona et al. (2020: 10): revision [99].

\section{- Genus Protocardiophorus Dolin, 1976}

Protocardiophorus Dolin, 1976: 71 [52]. Type species: Protocardiophorus ancestralis Dolin, 1976: 73 [52]. For more information, see Douglas [108] and Kundrata et al. [12].

Photocardiophorus: Dolin, 1980: legend to Figure 84 [53] [unavailable name, incorrect subsequent spelling not in prevailing usage; [129], Art. 33.3].

Remark. Species of this genus do not seem to be congeneric. What is more, the type material should be studied in order to confirm that they belong to Elateridae and not to other clicking elateroid lineages, especially Eucnemidae.

\section{Protocardiophorus ancestralis Dolin, 1976}

Protocardiophorus ancestralis Dolin, 1976: 73 [52]. (PIN).

Type material. Holotype, sex unknown, exoskeleton, compression fossil, No. 2066/2571

Fossil deposit/age. Kazakhstan: Karabastau Formation, Karatau, Mikhailovka; 166.1157.3 Ma (Jurassic).

Literature. Dolin (1976: 73): original description [52]; Dolin (1980: 78): key, additional specimen No. 2997/1973 [53]; Carpenter (1992: 305): generic catalogue [68]; Korneev and Cate (2005: 10): checklist [120]; Kundrata et al. (2020: 19): generic catalogue [12].

\section{Protocardiophorus jurassicus Dolin, 1980}

Protocardiophorus jurassicus Dolin, 1980: 78 [53].

Photocardiophorus [sic!] jurassicus: Dolin, 1980: legend to Figure 84 [53]. (PIN).

Type material. Holotype, sex unknown, exoskeleton, compression fossil, No. 2997/2020

Fossil deposit/age. Kazakhstan: Karabastau Formation, Karatau, Mikhailovka; 166.1157.3 Ma (Jurassic).

Literature. Dolin (1980: 78): original description [53]; Korneev and Cate (2005: 17): checklist [120].

Remark. Protocardiophorus jurassicus is morphologically similar to the species of $I d-$ iomerus which were transferred to Cerophytidae $[77,218]$.

\section{- Genus Pseudocardiophorites Dolin, 1976}

Pseudocardiophorites Dolin, 1976: 73 [52]. Type species: Pseudocardiophorites fragilis Dolin, 1976: 73 [52]. For more information, see Douglas [108] and Kundrata et al. [12].

Remark. This genus needs a revision since some species differ from the type species (and also from each other) in the body proportions, the shape and structure of thorax, 
etc. We cannot exclude the possibility that at least some species currently classified in this genus might belong to Eucnemidae.

\section{Pseudocardiophorites angustatus Dolin, 1980}

Pseudocardiophorites angustatus Dolin, 1980: 80 [53]. (PIN).

Type material. Holotype, sex unknown, exoskeleton, compression fossil, No. 2997/2019

Fossil deposit/age. Kazakhstan: Karabastau Formation, Karatau, Mikhailovka; 166.1157.3 Ma (Jurassic).

Literature. Dolin (1980: 80): original description [53]; Korneev and Cate (2005: 11): checklist [120].

\section{Pseudocardiophorites fragilis Dolin, 1976}

Pseudocardiophorites fragilis Dolin, 1976: 73 [52].

Cardiophorites [sic!] fragilis: Dolin, 1976: 72 [52] (figure legend).

Type material. Holotype, sex unknown, exoskeleton, compression fossil, No. 2554/688 (PIN).

Fossil deposit/age. Kazakhstan: Karabastau Formation, Karatau, Mikhailovka; 166.1157.3 Ma (Jurassic).

Literature. Dolin (1976: 73): original description [52]; Dolin (1980: 79): revision [53]; Carpenter (1992: 305): generic catalogue [68]; Korneev and Cate (2005: 10): checklist [120]; Kundrata et al. (2020: 19): generic catalogue [12].

\section{Pseudocardiophorites hayeki Dolin, 1976}

Pseudocardiophorites hayeki Dolin, 1976: 73 [52].

Cardiophorites [sic!] hayeki: Dolin, 1976: 72 [52] (figure legend).

Pseudocardiophorites hayekae: Dolin, 1980: 80 [53] [unavailable name, incorrect subsequent spelling not in prevailing usage; [129], Art. 33.4]. (PIN).

Type material. Holotype, sex unknown, exoskeleton, compression fossil, No. 2066/2930

Fossil deposit/age. Kazakhstan: Karabastau Formation, Karatau, Mikhailovka; 166.1157.3 Ma (Jurassic).

Literature. Dolin (1976: 73): original description [52]; Dolin (1980: 80): key [53]; Korneev and Cate (2005: 16): checklist [120].

\section{Pseudocardiophorites infractus Dolin, 1976}

Pseudocardiophorites infractus Dolin, 1976: 74 [52].

Cardiophorites [sic!] infractus Dolin, 1976: 72 [52] (figure legend).

Type material. Holotype, sex unknown, exoskeleton, compression fossil, No. 2239/1468 (PIN).

Fossil deposit/age. Kazakhstan: Karabastau Formation, Karatau, Mikhailovka; 166.1157.3 Ma (Jurassic).

Literature. Dolin (1976: 74): original description [52]; Dolin (1980: 80): key [53]; Korneev and Cate (2005: 17): checklist [120].

\section{Pseudocardiophorites quadricollis Dolin, 1976}

Pseudocardiophorites quadricollis Dolin, 1976: 74 [52].

Type material. Holotype, sex unknown, exoskeleton, compression fossil, No. 2239/1422 (PIN). Paratype, sex unknown, exoskeleton, compression fossil, No. 2239/1435 (PIN).

Fossil deposit/age. Kazakhstan: Karabastau Formation, Karatau, Mikhailovka (PIN collection 2239); 166.1-157.3 Ma (Jurassic).

Literature. Dolin (1976: 74): original description [52]; Dolin (1980: 80): key [53]; Korneev and Cate (2005: 22): checklist [120]. 


\section{- Genus Silicernius Heyden, 1859}

Silicernius Heyden, 1859: 6 [220]. Type species: Silicernius spectabilis Heyden, 1859: 6 [220]. For more information, see Kundrata et al. [12].

Remark. This genus might be related to Oxynopterini based on the body proportions and shapes of pronotum and elytra.

\section{Silicernius spectabilis Heyden, 1859}

Silicernius spectabilis Heyden, 1859: 6 [220].

Type material. Holotype, sex unknown, compression fossil (GPIBO).

Fossil deposit/age. Germany: Rott Formation; 28.4-23.03 Ma (Oligocene).

Literature. Heyden (1859: 6): original description [220]; Scudder (1885: 797): catalogue [22]; Scudder (1891: 580): catalogue [24]; Handlirsch (1907: 747): catalogue [127]; Hyslop (1921: 669): generic catalogue [111]; Kundrata et al. (2020: 19): generic catalogue [12].

Remark. This species resembles Oxynopterini in the broadened and almost roundly campaniform pronotum, with moderately long posterior angles, and elytron notably elongated and attenuate to apex.

\section{- Genus Sinoelaterium Ping, 1928}

Sinoelaterium Ping, 1928: 22 [40]. Type species: Sinoelaterium melanocolor Ping, 1928: 23 [40]. For more information, see Kundrata et al. [12].

Remark. This genus needs a thorough re-examination as it is not clear whether it belongs to Elateridae $[12,68]$. Some authors suggested that it might belong to Artematopodidae [221,222], but it also resembles Cerophytidae by the habitus, shape of antennae, and structure of head and thorax.

\section{Sinoelaterium melanocolor Ping, 1928}

Sinoelaterium melanocolor Ping, 1928: 23 [40].

Sinoelaterium melanovolor: Carpenter, 1992: 305 [68] [unavailable name, incorrect subsequent spelling not in prevailing usage; [129], Art. 33.3].

Type material. Holotype, sex unknown, exoskeleton, compression fossil, No. 2132 (W. H. Wong coll., Geological Survey of China [40]).

Fossil deposit/age. China: Liaoning Province, Yixian Formation, Beipiao City, Locality $228 ; 125.45-122.46 \mathrm{Ma}$ (Cretaceous).

Literature. Ping (1928: 23): original description [40]; Handlirsch (1938: 167, 169): catalogue [212]; Carpenter (1992: 305): generic catalogue [68]; Dolin and Nel (2002: 345): remark [82]; Dong and Huang (2009: 102): remark [80]; Dong and Huang (2011: 1227): remark [81]; Kundrata et al. (2020: 19): generic catalogue [12]; Muona et al. (2020: 11): remark [99].

\section{- Genus Tetraraphes Iablokoff-Khnzorian, 1961}

Tetraraphes Iablokoff-Khnzorian, 1961: 95 [47]. Type species: Tetraraphes ebersini Iablokoff-Khnzorian, 1961: 96 [47]. For more information, see Douglas [108] and Kundrata et al. [12].

\section{Tetraraphes ebersini Iablokoff-Khnzorian, 1961}

Tetraraphes ebersini Iablokoff-Khnzorian, 1961: 96 [47].

Type material. Holotype, sex unknown, exoskeleton, amber inclusion, No. 364/712 (PIN).

Fossil deposit/age. Baltic amber; 38.0-33.9 Ma (Eocene).

Literature. Iablokoff-Khnzorian (1961: 96): original description [47]; Larsson (1978: 153): catalogue [48]; Spahr (1981: 49): catalogue [49]; Keilbach (1982: 247): catalogue [133]; Carpenter (1992: 305): generic catalogue [68]; Alekseev (2013: 7): checklist [92]; Chang et al. 
(2010: 867): remark [86]; Kundrata et al. (2020: 19): generic catalogue [12].

\section{- Genus Turonelater Alekseev, 2011}

Turonelater Alekseev, 2011: 430 [75]. Type species: Turonelater giganteus Alekseev, 2011: 430 [75]. For more information, see Alekseev [75] and Kundrata et al. [12].

Remark. This genus probably belongs to Dendrometrinae based on the morphology of prothorax.

\section{Turonelater giganteus Alekseev, 2011}

Turonelater giganteus Alekseev, 2011: 430 [75].

Type material. Holotype, sex unknown, impression, No. 2383/252 (PIN).

Fossil deposit/age. Kazakhstan: Kzylorda region, Pond mudstone, Kzyl-Zhar; 93.989.8 Ma (Cretaceous).

Literature. Alekseev (2011: 430): original description [75]; Kundrata et al. (2020: 20): generic catalogue [12].

Remark. Turonelater giganteus has a broad pronotum, with arcuate sides, and moderately long and carinate posterior angles. Such pronotum can be found in the widely delimited Dendrometrinae, especially within Prosternini, Selatosomini, Dimini and Oxynopterini. This species might actually belong to Oxynopterini based on the large body size, the pronotum deeply arcuated anteriorly, and the prosternal lobe seemingly weakly developed.

\section{- Genus incertae sedis}

\section{Acmaeodera burmitina Cockerell, 1917}

Acmaeodera burmitina Cockerell, 1917: 323 [33].

Type material. Holotype, sex unknown, amber inclusion, No. PI In. 19,107 (BMNH). Fossil deposit/age. Myanmar: Burmese amber; 99.6-93.5 Ma (Cretaceous).

Literature. Cockerell (1917: 14): remark [as Elateridae] [32]; Cockerell (1917: 323): original description [as Buprestidae] [33]; Fletcher (1920: 987): remark [186]; Štys (1969: 357): remark [as Buprestidae] [223]; Zherikhin (1978: 114): remark [187]; Spahr (1981: 14): catalogue [as Buprestidae] [49]; Keilbach (1982: 248): checklist [as Buprestidae] [133]; Poinar (1992: 136): remark [as Buprestidae] [188]; Bellamy (1995: 175): review [as Elateridae] [224]; Ross (1998: 13) remark [225]; Ross and York (2000: 12): catalogue [as Elateridae] [189]; Bellamy (2008: 41): catalogue [as Elateridae] [226]; Ding et al. (2014: Table ES1): checklist [as Buprestidae] [227]; Peris and Háva (2016: 496): remark [as Elateridae] [190]; Otto (2019: 2): remark [as Elateridae] [96].

Remark. Although this species was described in the buprestid genus Acmaeodera Eschscholtz, 1829, it represents a member of Elateridae [32,33,189,224,226].

\section{Discussion}

In this study, we summarized information on all described fossil species in Elateridae. Altogether, 261 fossil species classified in 99 genera and nine subfamilies are currently listed in this family. Nevertheless, our results show that our knowledge of click-beetle palaeodiversity varies widely with respect to systematic, spatial and temporal elements.

The highest diversity of fossil Elateridae lies in the only exclusively fossil subfamily Protagrypninae $[12,51,53]$. It contains 94 species in 31 genera classified in four tribes (Table A1), which is more than a third of the described species diversity of fossil Elateridae. Not surprisingly, the next most diverse subfamilies include Agrypninae (13 genera/35 spp.), Dendrometrinae (11/35) and Elaterinae (13/29), which are three most species-rich extant click-beetle subfamilies based on numbers of described species $[1,228]$. The remaining subfamilies are represented only by a few species each. It should be noted, however, that another 50 species are currently considered incertae sedis, without a subfamily assignment (Table A1). 
Regarding the geographic origin of fossil click-beetle species, the highest diversity comes from Eurasian deposits. By far the richest locality is the famous Late Jurassic Karatau in Kazakhstan from which 100 species have been described in 29 genera [51-53]. Other, at least moderately rich localities, include the Jurassic Daohugou in Inner Mongolia of China (4 genera/4 spp.), the Lower Cretaceous Yixian Formation in northeastern China $(7 / 10)$ and the Zaza Formation of Baissa in Siberia (2/6), and the Miocene Shanwang Formation in eastern China (2/6). In Europe, the highest diversity of click-beetles has been described from Eocene Baltic amber, which has been redeposited from its original stratigraphic positions mainly in marine sediments and fluvial deposits, and contains the most diverse assemblage of fossil insects to date, including 17 click-beetle species classified in 16 genera $[47,48,229]$. Other important localities include the world-famous Eocene Grube Messel Pit (2 genera/11 spp.) and the Miocene Öhningen within the Upper FreshwaterMolasse Formation in Germany (8/10). The world-famous Florissant in Colorado, USA (Eocene) is the richest deposit in North America, with 38 click-beetle species classified in 17 genera $[28,29]$. Five species in five genera are known from the Eocene Green River Formation in USA, and four species in three genera were described from Mexican "Chiapas" amber, which is Miocene in age $[46,61]$. Several fossil click-beetle lineages were reported also from South America. However, the genera Babuskaya Martins-Neto and Gallego, 2009, Cardiosyne Martins-Neto and Gallego, 2006, and Gemelina Martins-Neto and Gallego, 2006 from the Mesozoic Argentinian deposits [71,72] were recently transferred from Elateridae to Coleoptera incertae sedis [12], and two Paleocene species were described based on elytra only, and their placement in Elateridae is dubious [35]. The Australian click-beetle fossil fauna includes five species in four genera from Mesozoic deposits; however, at least four species highly probably do not belong to Elateridae (see, e.g., Muona et al. [99]). Nevertheless, considering the relatively unique and rich extant Australian click-beetle fauna [228], as well as the fact that at least some lineages were among the early splits in the elaterid phylogeny $[5,11]$, a Mesozoic fossil record of that family in Australia would make sense. Indeed, Oberprieler et al. [98] reported a possible undescribed elaterid from the Jurassic Talbragar Fish Bed but this record needs further investigation. Thus far, there are no fossil Elateridae described from African deposits.

Our knowledge of the click-beetle fossil record should help us to better estimate the origin of the group and understand the evolutionary changes throughout its history. Based on the available data, it is possible that the Elateridae originated as early as the Triassic; however, this has to be confirmed by further research on the already described specimens and new material from Triassic deposits from around the globe. Currently, six described species are reported from the Triassic, most of them of highly doubtful family attribution, especially those from the Australian Blackstone Formation [39]. The highest diversity of fossil Elateridae is reported from the Jurassic (113 species/39 genera/five subfamilies), with the vast majority of lineages described from the rich Karatau deposit [51-53]. Only 24 species were described from Cretaceous localities, mainly from China and Russia, but we can expect that many more species will be discovered in Burmese amber in the near future. While 143 click-beetle species are recorded from the Mesozoic, only 118 described species are known from Cenozoic deposits. Most of them were from the Eocene Epoch, mainly due to the rich sources such as the North American Florissant Formation and European Baltic amber. It should be noted that all Mesozoic click-beetle species belong to fossil genera, i.e., those in which no extant representatives are included, with the apparently wrongly classified Burmese-amber species Elater burmitinus and "Acmaeodera" burmitina being exceptions $[32,33]$. On the other hand, slightly more than half of the elaterid genera known from the Paleogene (i.e., Paleocene, Eocene and Oligocene) also contain extant species, and among 12 genera known from the Neogene (Miocene) only two include exclusively fossil species.

Although our current study is the first comprehensive overview of described fossil species in Elateridae and is intended to serve as a solid basis for all future studies of the clickbeetle fossil record, it is an annotated catalogue rather than a taxonomic revision. Therefore, 
it must be treated with caution and interpreted carefully. Our results clearly show that the major problem with the click-beetle fossil record lies in the highly questionable family placement of many lineages, incorrectly interpreted morphological characters for fossil higher click-beetle taxa, and dubious or sometimes clearly erroneous generic assignments of many species across the whole classification of Elateridae. Incorrect identification of Mesozoic specimens can obscure our understanding of the origin of Elateridae and greatly affect the accuracy of the dating of phylogenetic trees in various studies. Click-beetle fossils from the rich Karatau deposit were used as one of the calibration points for a recent Coleoptera phylogeny [230], and Elaterophanes was used as an important calibration point in a dated molecular phylogenetic analysis of Elateroidea [231] or even of the whole of Coleoptera [232]. Kusy et al. [10] showed that analyses using different datasets, applied models and calibrations often come to different age estimates for the major splits as well as for the origin of bioluminescence in Elateroidea.

Detailed investigation of the family placement of most Mesozoic taxa currently assigned to Elateridae is crucial for our understanding of the origin, early evolution and past diversity of the group. One of the problems is that some of the oldest fossils are known only from a single elytron or its fragment and therefore, their placement even to a (super) family remains questionable [31,37,39]. Another problem is the uncertain family placement of many compression fossils from the Mesozoic Asian deposits, mainly from China and Karatau in Kazakhstan. The systematic placement of some Chinese click-beetles was already questioned in several $[86,233,234]$. Recently, Muona et al. [99] studied the Mesozoic clicking Elateroidea from Chinese deposits and discussed the external characters for recognizing Eucnemidae from other clicking elateroids, especially Elateridae. They showed that only about a third, i.e., 12 of 27, described fossil click-beetle species from China can be attributed to Elateridae with more or less certainty. One species was transferred to Throscidae, six to Eucnemidae, three could be either Elateridae or Eucnemidae based on the available characters, and five could not be studied due to the unavailability of the type material. As correctly pointed out by the authors [99], this drastically changed our view of the Mesozoic clicking elateroid fauna in China.

Moreover, a similar or even more dramatic situation may occur after the putative click-beetle taxa from the extremely rich Karatau deposit are re-examined in detail based on the type material. Dolin [51-53] focused on that deposit and reported from there an exceptionally high diversity of Elateridae, with 107 described species in 31 genera from five subfamilies. Interestingly, the vast majority species from Karatau are classified in the only fossil elaterid subfamily, Protagrypninae (Table A1). It was proposed by Dolin [51] for the earlier defined tribe Protagrypnini, which he originally described based on two genera from the Dzhil Formation in Kyrgyzstan and placed it in Agrypninae [50], and two other tribes, Desmatini and Hypnomorphini, from Karatau. He defined the subfamily based on the presence of longitudinal furrows (sutures) definining a medial field on the prosternum, a transverse suture on the mesoventrite, and the additional division of the apex of the radial cell on the hind wing [51,53]. However, it was evident even from the descriptions and illustrations that many of the species originally assigned to the click-beetle subfamily Protagrypninae may in fact represent some other clicking elateroid lineages. Indeed, Chang et al. [77] removed several species of one genus from Hypnomorphini to Cerophytidae, and many other species with potentially eucnemidor throscid-like characters should be re-examined (see Muona et al. [99] for extensive discussion on such characters, and remarks under various protagrypnine taxa in the overview of fossil species above). The diagnosis and monophyly of Protagrypninae are questionable, and especially problematic is the inclusion of Desmatini, which do not fully fit into the subfamily diagnosis as their representatives lack a clearly defined transverse suture on the mesoventrite [51,53]. They also have considerably broadened metacoxal plates, a character common in Eucnemidae ([86,234] but see [99]). Potential transfer of a number of taxa currently listed in Elateridae to Eucnemidae or Throscidae would make sense considering the earlier origin and longer evolutionary history of eucnemids, cerophytids 
and throscids $[230,231,235]$. Indeed, many recent studies confirm a high diversity of these families in the Mesozoic fossil record [96,99,218,233,234,236-240]. On the other hand, there might be some opposite cases. For example, Alekseev [74] described the monotypic genus Cretopoena from the Lower Cretaceous of Mongolia and attributed it to Eucnemidae; however, Li et al. [234] treated it as Elateriformia incertae sedis due to the lack of characters clearly separating Eucnemidae and Elateridae. Based on the general body shape and the structure of thorax this genus might indeed belong to Elateridae. Moreover, its thorax and elytra bear strong granulation which might be possible traces of scale-like setae typical for the Agrypnini. However, Cretopoena clearly differs from Agrypnini by its closed pronotosternal sutures and, therefore, its systematic position should be further investigated. Additionally, the suggested close relationship between Elateridae and recently discovered Mysteriomorphidae from the Cretaceous Burmese amber needs to be investigated using an analytical approach [241,242].

After the family placements of Mesozoic (and also younger) taxa are investigated in detail, it would be important to classify all fossil species to the proper genera and subfamilies. This will, however, be hampered not only by the lack of visible diagnostic characters on fossil specimens but also by the constantly changing definitions of the higher taxa and apparently unstable suprageneric classification of Elateridae $[1,6,11,228]$. The presence and character of the putative median plate-like structure on the prosternum laterally defined by furrows or sutures in Protagrypninae was discussed and questioned by Muona et al. [99] who concluded based on a study of fossil and extant specimens of clicking elateroids that it might be a place which is abruptly lower than the surrounding portions of the prosternum. The second important character, i.e., the transverse suture on the mesoventrite, which mainly defines Protagrypnini and Hypnomorphini, may in fact represent the line between the mesoventrite body and the depressions on the anterior edge of the mesoventrite and mesanepisternum, which are common in clicking elateroids and for which we use the terms "procoxal rests" [243] or "anterior articulating surface" [244]. The secondarily divided radial cell in the hind wing venation needs further investigation as this character is usually difficult to observe in compression fossils. Among the Cenozoic click-beetle fossils, those which are in the most urgent need of revision are species described by Scudder [19] and Wickham [27,28], especially those from the Green River and Florissant Formations [245], and species described by Heer from Öhningen [14], for which similarly inaccurate generic attributions were also reported in other beetle families [246,247]. Of special interest are the waste-basket genera such as Elater or Ctenicera. Further, maximum effort should be put into the study of the genera incertae sedis which include 50 species from various geological ages (Table A1), and also into description of the as-yet formally undescribed click-beetle fossils reported from various deposits $[229,248,249]$, as they may provide further information on the diversity of the main click-beetle lineages.

Last but not least, Elateridae students should pay special attention to the study of fossils included in amber deposits. Although the research of beetles (and other taxa) from various ambers is nowadays very popular $[92,188,189,250-252]$ and scientists were even able to describe within a short time span several new beetle families based on amber material [241,253-257], the diversity of click-beetles in fossilized plant resins has been highly understudied. A study of the Elateridae diversity in amber is of great importance due to the three-dimensional preservation of specimens which allows us to compare the fossil fauna with extant specimens in much greater detail than in the case of compression fossils $[188,229]$. With the use of modern techniques such as micro-CT, researchers are able to reconstruct the morphology of a particular beetle even when the imporant diagnostic characters are obscured by opaque bubbles or suboptimal body position $[94,242,258]$. Regarding the clickbeetle diversity in amber, only a few formally undescribed Elateridae were reported from Cretaceous Lebanese and Oligocene/Miocene Dominican ambers [54,95,188,253]; personal observations of authors]. Becker [46] and Zaragoza Caballero [61] described four species from the Miocene Mexican "Chiapas" amber. Cretaceous Burmese amber contains a high number of Elateridae from various lineages [249,259]; personal observations of authors] but 
only two species were described by Cockerell [33] (although one originally in Bupestidae) and a single species by Otto [96]. Elateridae were among the most-represented beetle families in Eocene Baltic amber [30,48]; hence it is not surprising that most click-beetle species were described from that amber, mainly due to the work by Iablokoff-Khnzorian [47]. However, the vast majority of click-beetles known from all ambers remain undescribed.

\section{Conclusions}

An understanding of the origin, evolution, and past diversity of click-beetles is hampered by the lack of detailed knowledge on their fossil record. We summarized the current knowledge on all described fossil species in Elateridae, and assessed each species based on its description and available illustrations to conclude whether its position in Elateridae and its generic attribution can be considered reliable or not. Our results suggest that the Triassic records based largely on isolated elytra are mostly dubious and may belong to different beetle families, and numerous Jurassic and Cretaceous lineages currently listed in Elateridae might belong to Eucnemidae or Throscidae. The vast majority of the fossil click-beetle species are in urgent need of revision, and the incertae sedis genera should be investigated to correctly assign them to subfamilies and tribes. We can expect many more lineages to be discovered mainly from the more and more intensively studied amber inclusions, especially from Eocene Baltic amber and Cretaceous Burmese amber, which both include a relatively high proportion of elaterid fossils.

Author Contributions: Conceptualization, R.K.; investigation, R.K., G.P., A.S.P. and J.H.; writingoriginal draft, R.K. and G.P.; supervision, R.K.; project administration, R.K.; writing-review and editing, R.K., G.P., A.S.P. and J.H.; funding acquisition, R.K., J.H. and A.S.P. Results of this study were partly used in the unpublished bachelor thesis of G.P. supervised by R.K. All authors have read and agreed to the published version of the manuscript.

Funding: This research was funded by the internal grant of the Palacky University nr. IGA_PrF_2021_019 (to R.K. and J.H.), the Moscow State University grant for leading scientific schools “Depository of the living systems" in frame of the MSU development program, and the Moscow State University research project No. AAAA-A16-116021660095-7 (both to A.S.P.).

Institutional Review Board Statement: Not applicable.

Data Availability Statement: Not applicable.

Acknowledgments: We thank following colleagues for providing literature, photographs and/or useful information concerning this work and fossil Elateridae: David Peris (Spain), Evgeny V. Yan (PIN, Russia), Karol Szawaryn (Poland), Hume Douglas and Patrice Bouchard (Canada), Yanchen Zhao, Lu Qiu (China), Andris Bukejs (Latvia), Václav Dušánek (Czech Republic), Christel and Hans Werner Hoffeins (Germany), Maxwell V. L. Barclay and Claire Mellish (BMNH), Jyrki Muona (Finland), Christoph Schindler (Germany), Janine Mazenauer (Switzerland), Torsten Wappler (Germany), Mark G. Volkovitsh (ZIN, Russia), and Mónica M. Solórzano Kraemer (Germany). We are grateful to Matthew L. Gimmel (USA) for proofreading the earlier version of the manuscript, and to two anonymous reviewers for their valuable comments. We thank Melissa Aja and the Museum of Comparative Zoology and Harvard University (MCZ) for permission to use the photograph of Oxygonus primus, Keita Matsumoto and the Natural History Museum in London (BMNH) for permission to use the photograph of Megapenthes voigti, and Craig M. Brabant and Daniel K. Young (WIRC) for the loan of the paratype of Cretopityobius pankowskiorum.

Conflicts of Interest: R.K. and A.S.P. are Editors of Insects. The authors declare no other conflict of interest. The funders had no role in the design of the study; in the collection, analyses, or interpretation of data; in the writing of the manuscript, or in the decision to publish the results. 


\section{Appendix A}

Table A1. Overview of fossil Elateridae. Genera marked with an asterisk $\left(^{*}\right)$ also contain recent species. Geographic origin includes country and formation when available. Period/Epoch: T, Triassic; J, Jurassic; C, Cretaceous; P, Paleocene; E, Eocene; O, Oligocene; M, Miocene.

\begin{tabular}{|c|c|c|c|}
\hline Subfamily, Tribe, Genus & Species & Geographic Origin & Age (Ma), Period/Epoch \\
\hline \multicolumn{4}{|l|}{ Agrypninae } \\
\hline \multicolumn{4}{|l|}{ Agrypnini } \\
\hline Adelocera Latreille, $1829 *$ & $\begin{array}{c}\text { A. perantiqua Cockerell and } \\
\text { LeVeque, } 1931\end{array}$ & USA: Green River & $50.3-46.2(\mathrm{E})$ \\
\hline \multirow[t]{2}{*}{ Ageratus Dolin, 1980} & A. delicatus Dolin, 1980 & Kazakhstan: Karabastau & $166.1-157.3(\mathrm{~J})$ \\
\hline & A. p onomarenkoi Dolin, 1980 & Kazakhstan: Karabastau & $166.1-157.3(\mathrm{~J})$ \\
\hline Agrypnus Eschscholtz, 1829 * & A. exhumatus (Wickham, 1916) & USA: Florissant & $37.2-33.9(\mathrm{E})$ \\
\hline Compsoderus Dolin, 1980 & C. priscus Dolin, 1980 & Kazakhstan: Karabastau & $166.1-157.3(\mathrm{~J})$ \\
\hline \multirow[t]{3}{*}{ Lacon Laporte, 1838 * } & L. granulatus (Heer, 1847) & $\begin{array}{c}\text { Germany: Upper } \\
\text { Freshwater-Molasse }\end{array}$ & 12.7-11.608 (M) \\
\hline & L. jungi (Piton, 1940) & France: Menat & $61.6-59.2(\mathrm{P})$ \\
\hline & L. primordialis Heer, 1847 & $\begin{array}{l}\text { Germany: Upper } \\
\text { Freshwater-Molasse }\end{array}$ & 12.7-11.608 (M) \\
\hline \multirow[t]{7}{*}{ Litholacon Dolin, 1980} & L. conicicollis Dolin, 1980 & Kazakhstan: Karabastau & $166.1-157.3(\mathrm{~J})$ \\
\hline & L. derumpens Dolin, 1980 & Kazakhstan: Karabastau & $166.1-157.3(\mathrm{~J})$ \\
\hline & L. exilis Dolin, 1980 & Kazakhstan: Karabastau & $166.1-157.3(\mathrm{~J})$ \\
\hline & L. major Dolin, 1980 & Kazakhstan: Karabastau & $166.1-157.3(\mathrm{~J})$ \\
\hline & L. panphilovi Dolin, 1980 & Kazakhstan: Karabastau & $166.1-157.3(\mathrm{~J})$ \\
\hline & L. ohiri Dolin, 1980 & Kazakhstan: Karabastau & $166.1-157.3(\mathrm{~J})$ \\
\hline & L. petrorsus Dolin, 1980 & Kazakhstan: Karabastau & $166.1-157.3(\mathrm{~J})$ \\
\hline \multirow[t]{13}{*}{ Macropunctum Tröster, 1991} & M. angulosum Tröster 1999 & Germany: Messel & 48.6-40.4 (E) \\
\hline & M. angustiscutellum Tröster, 1994 & Germany: Messel & $48.6-40.4(\mathrm{E})$ \\
\hline & M. densipunctum Wappler, 2003 & Germany: Eifel & $48.6-40.4(\mathrm{E})$ \\
\hline & M. eckfeldi Tröster, 1992 & Germany: Eifel & $48.6-40.4(\mathrm{E})$ \\
\hline & M. eоcaenicum (Meunier, 1921) & Germany: Messel & $48.6-40.4(\mathrm{E})$ \\
\hline & M. latiscutellum Tröster & Germany: Messel & $48.6-40.4(\mathrm{E})$ \\
\hline & M. messelense Tröster, 1991 & Germany: Messel & $48.6-40.4(\mathrm{E})$ \\
\hline & M. meunieri Tröster, 1991 & Germany: Messel & $48.6-40.4(\mathrm{E})$ \\
\hline & M. minutum (Meunier, 1921) & Germany: Messel & $48.6-40.4(\mathrm{E})$ \\
\hline & M. promptum (Meunier, 1921) & Germany: Messel & $48.6-40.4(\mathrm{E})$ \\
\hline & M. rebugense Tröster, 1994 & Germany: Messel & $48.6-40.4(\mathrm{E})$ \\
\hline & M. rossi Alekseev, 2019 & United Kingdom: Bouldnor & $38.0-33.9(\mathrm{E})$ \\
\hline & M. senckenbergi Tröster, 1994 & Germany: Messel & $48.6-40.4(\mathrm{E})$ \\
\hline $\begin{array}{c}\text { Plagioraphes } \\
\text { Iablokoff-Khnzorian, } 1961\end{array}$ & $\begin{array}{l}\text { P. fasciatus Iablokoff-Khnzorian, } \\
1961\end{array}$ & Europe: Baltic amber & $38.0-33.9(\mathrm{E})$ \\
\hline
\end{tabular}


Table A1. Cont.

\begin{tabular}{|c|c|c|c|}
\hline Subfamily, Tribe, Genus & Species & Geographic Origin & Age (Ma), Period/Epoch \\
\hline \multicolumn{4}{|l|}{ Cryptocardiini } \\
\hline Cryptocardius Dolin, 1980 & C. mirabilis Dolin, 1980 & Kazakhstan: Karabastau & $166.1-157.3(\mathrm{~J})$ \\
\hline \multicolumn{4}{|l|}{ Hemirhipini } \\
\hline Alaus Eschscholtz, 1829* & A. spectabilis (Heer, 1865) & $\begin{array}{l}\text { Germany: Upper } \\
\text { Freshwater-Molasse }\end{array}$ & $12.7-11.608(\mathrm{M})$ \\
\hline \multicolumn{4}{|l|}{ Oophorini } \\
\hline $\begin{array}{l}\text { Monocrepidius Eschscholtz, } \\
1829 *\end{array}$ & M. dubiosus Wickham, 1916 & USA: Florissant & $37.2-33.9(\mathrm{E})$ \\
\hline \multicolumn{4}{|l|}{ Pseudomelanactini } \\
\hline \multirow[t]{2}{*}{ Lanelater Arnett, 1952 * } & L. nicoleae Wappler, 2003 & Germany: Eifel & $48.6-40.4(\mathrm{E})$ \\
\hline & L. verae Tröster, 1993 & Germany: Messel & $48.6-40.4(\mathrm{E})$ \\
\hline \multicolumn{4}{|l|}{ Pyrophorini } \\
\hline Eopyrophorus Haupt, 1950 & E. mixtus Haupt, 1950 & Germany: Geiseltal & $47.8-41.3(\mathrm{E})$ \\
\hline \multicolumn{4}{|l|}{ Cardiophorinae } \\
\hline \multirow[t]{8}{*}{$\begin{array}{l}\text { Cardiophorus Eschscholtz, } \\
1829 *\end{array}$} & C. braunii Heer, 1847 & $\begin{array}{l}\text { Germany: Upper } \\
\text { Freshwater-Molasse }\end{array}$ & $12.7-11.608(\mathrm{M})$ \\
\hline & C. cockerelli Wickham, 1916 & USA: Florissant & $37.2-33.9(\mathrm{E})$ \\
\hline & C. deprivatus Wickham, 1916 & USA: Florissant & $37.2-33.9(\mathrm{E})$ \\
\hline & C. exhumatus Cockerell, 1926 & USA: Green River & $50.3-46.2(\mathrm{E})$ \\
\hline & C. florissantensis Wickham, 1916 & USA: Florissant & $37.2-33.9(\mathrm{E})$ \\
\hline & C. lithographus Wickham, 1916 & USA: Florissant & $37.2-33.9(\mathrm{E})$ \\
\hline & C. requiescens Wickham, 1916 & USA: Florissant & $37.2-33.9(\mathrm{E})$ \\
\hline & $\begin{array}{c}\text { C. yatsenkokhmelevskyi } \\
\text { Iablokoff-Khnzorian, } 1961\end{array}$ & Europe: Baltic amber & $38.0-33.9(\mathrm{E})$ \\
\hline Horistonotus Candèze, 1860 * & H. coloradensis Wickham, 1916 & USA: Florissant & $37.2-33.9(\mathrm{E})$ \\
\hline \multicolumn{4}{|l|}{ Dendrometrinae } \\
\hline \multicolumn{4}{|l|}{ Dendrometrini } \\
\hline \multicolumn{4}{|l|}{ Athous Eschscholtz, $1829 *$} \\
\hline $\begin{array}{l}\text { Subg. Athousiomorphus } \\
\text { Iablokoff-Khnzorian, } 1961\end{array}$ & $\begin{array}{l}\text { A. (A.) olgae Iablokoff-Khnzorian, } \\
1961\end{array}$ & Europe: Baltic amber & $38.0-33.9(\mathrm{E})$ \\
\hline \multirow[t]{4}{*}{ Subg. incertae sedis } & A. contusus Wickham, 1916 & USA: Florissant & $37.2-33.9(\mathrm{E})$ \\
\hline & A. fractus Wickham, 1916 & USA: Florissant & $37.2-33.9(\mathrm{E})$ \\
\hline & A. holmgreni (Heer, 1870) & Norway: Firkanten & $66.0-59.2(\mathrm{P})$ \\
\hline & A. lethalis Wickham, 1916 & USA: Florissant & $37.2-33.9(\mathrm{E})$ \\
\hline \multicolumn{4}{|l|}{ Limonius Eschscholtz, 1829 * } \\
\hline $\begin{array}{c}\text { Subg. Paralimonius } \\
\text { Iablokoff-Khnzorian, } 1961\end{array}$ & $\begin{array}{c}\text { L.(P.) barovskyi } \\
\text { Iablokoff-Khnzorian, } 1961\end{array}$ & Europe: Baltic amber & $38.0-33.9(\mathrm{E})$ \\
\hline Subg. incertae sedis & L. aboriginalis Wickham, 1916 & USA: Florissant & $37.2-33.9(\mathrm{E})$ \\
\hline
\end{tabular}


Table A1. Cont.

\begin{tabular}{|c|c|c|c|}
\hline Subfamily, Tribe, Genus & Species & Geographic Origin & Age (Ma), Period/Epoch \\
\hline & L. florissantensis Wickham, 1916 & USA: Florissant & $37.2-33.9(\mathrm{E})$ \\
\hline & L. impunctus Scudder, 1895 & Canada: Allenby & $56.0-47.8(\mathrm{E})$ \\
\hline & L. optabilis Heer, 1847 & $\begin{array}{l}\text { Germany: Upper } \\
\text { Freshwater-Molasse }\end{array}$ & $12.7-11.608(\mathrm{M})$ \\
\hline & L. praecursor Wickham, 1916 & USA: Florissant & $37.2-33.9(\mathrm{E})$ \\
\hline & L. shoshonis Wickham, 1916 & USA: Florissant & $37.2-33.9(\mathrm{E})$ \\
\hline & L. volans Wickham, 1916 & USA: Florissant & $37.2-33.9(\mathrm{E})$ \\
\hline \multicolumn{4}{|l|}{ Dimini } \\
\hline Alaodima Dolin, 1980 & A. grandis Dolin, 1980 & Kazakhstan: Karabastau & $166.1-157.3(\mathrm{~J})$ \\
\hline \multicolumn{4}{|l|}{ Hypnoidini } \\
\hline Ligmargus Stibick, 1976 * & L. terrestris (Scudder, 1879) & Canada: Nicola river & $56.0-47.8(\mathrm{E})$ \\
\hline \multicolumn{4}{|l|}{ Oxynopterini } \\
\hline Campsosternus Latreille, 1834 * & C. atavus Deichmüller, 1881 & Czech Republic: Kučlín & $37.2-33.9(\mathrm{E})$ \\
\hline Melanactes LeConte, 1853 * & M. cockerelli Wickham, 1908 & USA: Florissant & $37.2-33.9(\mathrm{E})$ \\
\hline \multicolumn{4}{|l|}{ Prosternini } \\
\hline \multirow[t]{10}{*}{ Ctenicera Latreille, 1829 * } & C. emblemoelytra (Zhang, 1989) & China: Shanwang & 20.44-15.97 (M) \\
\hline & C. euprepes (Zhang et al., 1994) & China: Shanwang & 20.44-15.97 (M) \\
\hline & C. granulicollis (Wickham, 1908) & USA: Florissant & $37.2-33.9(\mathrm{E})$ \\
\hline & C. primitiva (Wickham, 1908) & USA: Florissant & $37.2-33.9(\mathrm{E})$ \\
\hline & C. prophetica (Wickham, 1916) & USA: Florissant & $37.2-33.9(\mathrm{E})$ \\
\hline & C. restructa (Wickham, 1916) & USA: Florissant & $37.2-33.9(\mathrm{E})$ \\
\hline & C. sincera (Zhang et al., 1994) & China: Shanwang & 20.44-15.97 (M) \\
\hline & C. submersa (Wickham, 1916) & USA: Florissant & $37.2-33.9(\mathrm{E})$ \\
\hline & C. sutor (Heer, 1847) & $\begin{array}{l}\text { Germany: Upper } \\
\text { Freshwater-Molasse }\end{array}$ & 12.7-11.608 (M) \\
\hline & C. velata (Scudder, 1876) & USA: Green River & $50.3-46.2(\mathrm{E})$ \\
\hline \multirow[t]{3}{*}{ Eanus LeConte, $1861 *$} & E. exanimatus (Wickham, 1916) & USA: Florissant & $37.2-33.9(\mathrm{E})$ \\
\hline & E. heeri (Wickham, 1916) & USA: Florissant & $37.2-33.9(\mathrm{E})$ \\
\hline & E. laevissimus (Wickham, 1916) & USA: Florissant & $37.2-33.9(\mathrm{E})$ \\
\hline \multirow[t]{2}{*}{ Oxygonus LeConte, 1863 * } & O. mortuus Scudder, 1876 & USA: Green River & $50.3-46.2(\mathrm{E})$ \\
\hline & O. primus Wickham, 1916 & USA: Florissant & $37.2-33.9(\mathrm{E})$ \\
\hline \multicolumn{4}{|l|}{ Selatosomini } \\
\hline Selatosomus Stephens, 1830 * & S. miegi Theobald, 1937 & Germany: Middle Member & $33.9-28.4(\mathrm{O})$ \\
\hline \multicolumn{4}{|l|}{ Semiotini } \\
\hline \multirow[t]{2}{*}{ Semiotus Eschscholtz 1829 * } & S. ehrenswaerdi (Heer, 1870) & Norway: Firkanten & $66.0-59.2(\mathrm{P})$ \\
\hline & S. menatensis Piton, 1940 & France: Menat & $61.6-59.2(\mathrm{P})$ \\
\hline
\end{tabular}


Table A1. Cont.

\begin{tabular}{|c|c|c|c|}
\hline Subfamily, Tribe, Genus & Species & Geographic Origin & Age (Ma), Period/Epoch \\
\hline \multicolumn{4}{|l|}{ Elaterinae } \\
\hline \multicolumn{4}{|l|}{ Agriotini } \\
\hline \multirow[t]{3}{*}{ Agriotes Eschscholtz, 1829 * } & A. comminutus Wickham, 1916 & USA: Florissant & $37.2-33.9(\mathrm{E})$ \\
\hline & A. nearcticus Wickham, 1916 & USA: Florissant & $37.2-33.9(\mathrm{E})$ \\
\hline & A. succiniferus Becker, 1963 & Mexico: Chiapas amber & 23.03-15.97 (M) \\
\hline \multicolumn{4}{|l|}{ Ampedini } \\
\hline \multicolumn{4}{|l|}{ Ampedus Dejean, 1833 * } \\
\hline $\begin{array}{l}\text { Subg. Octamenogonoides } \\
\text { Iablokoff-Khnzorian, } 1961\end{array}$ & $\begin{array}{c}\text { A. (O.) gebleri } \\
\text { Iablokoff-Khnzorian, } 1961\end{array}$ & Europe: Baltic amber & 38.0-33.9 (E) \\
\hline Subg. Ampedus Dejean, 1833 * & A. seyfriedii Heer, 1847 & $\begin{array}{l}\text { Germany: Upper } \\
\text { Freshwater-Molasse }\end{array}$ & 12.7-11.608 (M) \\
\hline Ischnodes Germar, $1844^{*}$ & I. gracilis Heer, 1847 & $\begin{array}{l}\text { Germany: Upper } \\
\text { Freshwater-Molasse }\end{array}$ & $12.7-11.608(\mathrm{M})$ \\
\hline \multicolumn{4}{|l|}{ Elaterini } \\
\hline $\begin{array}{c}\text { Diaraphes Iablokoff-Khnzorian, } \\
1961\end{array}$ & $\begin{array}{c}\text { D. kozhantshikovi } \\
\text { Iablokoff-Khnzorian, } 1961\end{array}$ & Europe: Baltic amber & $38.0-33.9(\mathrm{E})$ \\
\hline \multirow[t]{10}{*}{ Elater Linnaeus, 1758 * } & E. asmodeus Zhang, 1989 & China: Shanwang & $20.44-15.97(\mathrm{M})$ \\
\hline & E. berryi Wickham, 1929 & USA: Cockfield & $41.3-38.0(\mathrm{E})$ \\
\hline & E. burmitinus Cockerell, 1917 & Myanmar: Burmese amber & $99.6-93.5(\mathrm{C})$ \\
\hline & E. canabinus Zhang, 1989 & China: Shanwang & $20.44-15.97(\mathrm{M})$ \\
\hline & E. florissantensis Wickham, 1916 & USA: Florissant & $37.2-33.9(\mathrm{E})$ \\
\hline & E. mitrus Zhang, 1989 & China: Shanwang & $20.44-15.97(\mathrm{M})$ \\
\hline & E. naumanni Giebel, 1856 & Europe: Baltic amber & $38.0-33.9(\mathrm{E})$ \\
\hline & E. rohweri Wickham, 1916 & USA: Florissant & $37.2-33.9(\mathrm{E})$ \\
\hline & E. scudderi Wickham, 1916 & USA: Florissant & $37.2-33.9(\mathrm{E})$ \\
\hline & E. wisniowskii Lomnicki, 1902 & Ukraine: Bashkev & 13.65-12.7 (M) \\
\hline $\begin{array}{l}\text { Elatron Iablokoff-Khnzorian, } \\
1961\end{array}$ & $\begin{array}{l}\text { E. semenovi Iablokoff-Khnzorian, } \\
1961\end{array}$ & Europe: Baltic amber & $38.0-33.9(\mathrm{E})$ \\
\hline $\begin{array}{c}\text { Holopleurus } \\
\text { Iablokoff-Khnzorian, } 1961\end{array}$ & $\begin{array}{l}\text { H. succineus Iablokoff-Khnzorian, } \\
1961\end{array}$ & Europe: Baltic amber & $38.0-33.9(\mathrm{E})$ \\
\hline $\begin{array}{c}\text { Orthoraphes } \\
\text { Iablokoff-Khnzorian, } 1961\end{array}$ & $\begin{array}{l}\text { O. reichardti Iablokoff-Khnzorian, } \\
1961\end{array}$ & Europe: Baltic amber & $38.0-33.9(\mathrm{E})$ \\
\hline \multicolumn{4}{|l|}{ Megapenthini } \\
\hline Abelater Fleutiaux, $1947^{*}$ & A. succineus Schimmel, 2005 & Europe: Baltic amber & $38.0-33.9(\mathrm{E})$ \\
\hline \multirow[t]{3}{*}{$\begin{array}{c}\text { Megapenthes Kiesenwetter, } \\
1858 \text { * }\end{array}$} & M. groehni Schimmel, 2005 & Europe: Baltic amber & $38.0-33.9(\mathrm{E})$ \\
\hline & M. primaevus Wickham, 1916 & USA: Florissant & $37.2-33.9(\mathrm{E})$ \\
\hline & M. voigti Schimmel, 2005 & Europe: Baltic amber & $38.0-33.9(\mathrm{E})$ \\
\hline
\end{tabular}


Table A1. Cont.

\begin{tabular}{|c|c|c|c|}
\hline Subfamily, Tribe, Genus & Species & Geographic Origin & Age (Ma), Period/Epoch \\
\hline \multicolumn{4}{|l|}{ Physorhinini } \\
\hline \multirow[t]{2}{*}{ Anchastus LeConte, 1853 * } & A. diluvialis Wickham, 1916 & USA: Florissant & $37.2-33.9(\mathrm{E})$ \\
\hline & A. eruptus Wickham, 1916 & USA: Florissant & $37.2-33.9(\mathrm{E})$ \\
\hline \multicolumn{4}{|l|}{ Synaptini } \\
\hline \multirow[t]{2}{*}{ Glyphonyx Candèze, 1863 * } & $\begin{array}{l}\text { G. chiapasensis Zaragoza } \\
\text { Caballero, } 1990\end{array}$ & Mexico: Chiapas amber & 23.03-15.97 (M) \\
\hline & G. punctatus Becker, 1963 & Mexico: Chiapas amber & 23.03-15.97 (M) \\
\hline \multicolumn{4}{|l|}{ Elaterinae incertae sedis } \\
\hline $\begin{array}{c}\text { Crioraphes } \\
\text { Iablokoff-Khnzorian, } 1961\end{array}$ & $\begin{array}{l}\text { C. rohdendorfi Iablokoff-Khnzorian, } \\
\qquad 1961\end{array}$ & Europe: Baltic amber & $38.0-33.9(\mathrm{E})$ \\
\hline \multicolumn{4}{|l|}{ Lissominae } \\
\hline \multicolumn{4}{|l|}{ Lissomini } \\
\hline Lissomus Dalman, 1824 * & L. taxodii (Heer, 1870) & Norway: Firkanten & $66.0-59.2(\mathrm{P})$ \\
\hline \multicolumn{4}{|l|}{ Protelaterini } \\
\hline Baltelater Kundrata et al., 2020 & $\begin{array}{l}\text { B. bipectinatus Kundrata et al., } \\
\qquad 2020\end{array}$ & Europe: Baltic amber & 38.0-33.9 (E) \\
\hline \multicolumn{4}{|l|}{ Negastriinae } \\
\hline \multirow[t]{2}{*}{ Ganestrius Dolin, 1976} & G. elongatus Dolin, 1976 & Kazakhstan: Karabastau & $166.1-157.3(\mathrm{~J})$ \\
\hline & G. stibicki Dolin, 1976 & Kazakhstan: Karabastau & $166.1-157.3(\mathrm{~J})$ \\
\hline \multirow[t]{2}{*}{ Paradonus Stibick, 1971 * } & $\begin{array}{c}\text { Paradonus exterminatus (Wickham, } \\
\text { 1916) }\end{array}$ & USA: Florissant & $37.2-33.9(\mathrm{E})$ \\
\hline & $\begin{array}{c}\text { Paradonus hesperus (Wickham, } \\
\text { 1916) }\end{array}$ & USA: Florissant & $37.2-33.9(\mathrm{E})$ \\
\hline Protoquasimus Dolin, 1976 & P. brevicollis Dolin, 1976 & Kazakhstan: Karabastau & $166.1-157.3(\mathrm{~J})$ \\
\hline \multicolumn{4}{|l|}{ Omalisinae } \\
\hline $\begin{array}{c}\text { Jantarokrama Kirejtshuk and } \\
\text { Kovalev, } 2015\end{array}$ & $\begin{array}{l}\text { J. utilis Kirejtshuk and Kovalev, } \\
2015\end{array}$ & Europe: Baltic amber & $38.0-33.9(\mathrm{E})$ \\
\hline \multicolumn{4}{|l|}{ Pityobiinae } \\
\hline Cretopityobius Otto, 2019 & C. pankowskiorum Otto, 2019 & Myanmar: Burmese amber & $99.6-93.5(\mathrm{C})$ \\
\hline \multicolumn{4}{|l|}{ Protagrypninae } \\
\hline \multicolumn{4}{|l|}{ Desmatini } \\
\hline Desmatinus Chang et al., 2010 & D. cognatus Chang et al., 2010 & China: Yixian & $125.45-122.46(\mathrm{C})$ \\
\hline \multirow[t]{5}{*}{ Desmatus Dolin, 1975} & D. affinis Dolin, 1975 & Kazakhstan: Karabastau & $166.1-157.3(\mathrm{~J})$ \\
\hline & D. beckeri Dolin, 1975 & Kazakhstan: Karabastau & $166.1-157.3(\mathrm{~J})$ \\
\hline & D. lapidarius Dolin, 1975 & Kazakhstan: Karabastau & $166.1-157.3(\mathrm{~J})$ \\
\hline & $\begin{array}{l}\text { D. ponomarenkoi (Chang et al., } \\
\text { 2009) }\end{array}$ & China: Jiulongshan & $166.1-157.3(\mathrm{~J})$ \\
\hline & D. protensus Dolin, 1980 & Kazakhstan: Karabastau & $166.1-157.3(\mathrm{~J})$ \\
\hline
\end{tabular}


Table A1. Cont.

\begin{tabular}{|c|c|c|c|}
\hline Subfamily, Tribe, Genus & Species & Geographic Origin & Age (Ma), Period/Epoch \\
\hline Plesiorhaphes Dolin, 1980 & P. scaber Dolin, 1980 & Kazakhstan: Karabastau & $166.1-157.3(\mathrm{~J})$ \\
\hline \multicolumn{4}{|l|}{ Hypnomorphini } \\
\hline \multirow[t]{2}{*}{ Abrotus Dolin, 1980} & A. reconditus Dolin, 1980 & Kazakhstan: Karabastau & $166.1-157.3(\mathrm{~J})$ \\
\hline & A. sepultus Dolin, 1980 & Kazakhstan: Karabastau & $166.1-157.3(\mathrm{~J})$ \\
\hline \multirow[t]{3}{*}{ Adiagnostus Dolin, 1980} & A. ambiguus Dolin, 1980 & Kazakhstan: Karabastau & $166.1-157.3(\mathrm{~J})$ \\
\hline & A. cardiophorinus Dolin, 1980 & Kazakhstan: Karabastau & $166.1-157.3(\mathrm{~J})$ \\
\hline & A. minutulus Dolin, 1980 & Kazakhstan: Karabastau & $166.1-157.3(\mathrm{~J})$ \\
\hline \multirow[t]{10}{*}{ Codemus Dolin, 1980} & C. alatus Dolin, 1980 & Kazakhstan: Karabastau & $166.1-157.3(\mathrm{~J})$ \\
\hline & C. carinatus Dolin, 1980 & Kazakhstan: Karabastau & $166.1-157.3(\mathrm{~J})$ \\
\hline & C. jejunus Dolin, 1980 & Kazakhstan: Karabastau & $166.1-157.3(\mathrm{~J})$ \\
\hline & C. martynovi Dolin, 1980 & Kazakhstan: Karabastau & $166.1-157.3(\mathrm{~J})$ \\
\hline & C. micros Dolin, 1980 & Kazakhstan: Karabastau & $166.1-157.3(\mathrm{~J})$ \\
\hline & C. quadricolis Dolin, 1980 & Kazakhstan: Karabastau & $166.1-157.3(\mathrm{~J})$ \\
\hline & C. sharovi Dolin, 1980 & Kazakhstan: Karabastau & $166.1-157.3(\mathrm{~J})$ \\
\hline & C. synaptoides Dolin, 1980 & Kazakhstan: Karabastau & $166.1-157.3(\mathrm{~J})$ \\
\hline & C. teres Dolin, 1980 & Kazakhstan: Karabastau & $166.1-157.3(\mathrm{~J})$ \\
\hline & C. zherichini Dolin, 1980 & Kazakhstan: Karabastau & $166.1-157.3(\mathrm{~J})$ \\
\hline \multirow[t]{2}{*}{ Dolinelater Huber et al., 2017} & D. asperatus (Dolin, 1980) & Kazakhstan: Karabastau & $166.1-157.3(\mathrm{~J})$ \\
\hline & D. singularis (Dolin, 1980) & Kazakhstan: Karabastau & $166.1-157.3(\mathrm{~J})$ \\
\hline \multirow[t]{3}{*}{ Elaterophanes Handlirsch, 1906} & E. acutus Cockerell, 1916 & $\begin{array}{l}\text { United Kingdom: Wainlode } \\
\text { Cliff }\end{array}$ & $208.5-201.3(\mathrm{~T})$ \\
\hline & E. regius Whalley, 1985 & $\begin{array}{l}\text { United Kingdom: } \\
\text { Charmouth Mudstone }\end{array}$ & $196.5-189.6(\mathrm{~J})$ \\
\hline & E. vetustus (Brodie, 1845) & United Kingdom: Lilstock & 208.5-201.3 (T) \\
\hline Graciolacon Dolin, 1980 & G. aeternus Dolin, 1980 & Kazakhstan: Karabastau & $166.1-157.3(\mathrm{~J})$ \\
\hline \multirow[t]{4}{*}{ Hypnomorphoides Dolin, 1980} & H. angularis Dolin, 1980 & Kazakhstan: Karabastau & $166.1-157.3(\mathrm{~J})$ \\
\hline & H. catachtonius Dolin, 1980 & Kazakhstan: Karabastau & $166.1-157.3(\mathrm{~J})$ \\
\hline & H. latus Dolin, 1980 & Kazakhstan: Karabastau & $166.1-157.3(\mathrm{~J})$ \\
\hline & H. procerulus Dolin, 1980 & Kazakhstan: Karabastau & $166.1-157.3(\mathrm{~J})$ \\
\hline \multirow[t]{11}{*}{ Hypnomorphus Dolin, 1975} & H. aemulus Dolin, 1975 & Kazakhstan: Karabastau & $166.1-157.3(\mathrm{~J})$ \\
\hline & H. angulosus Dolin, 1980 & Kazakhstan: Karabastau & $166.1-157.3(\mathrm{~J})$ \\
\hline & H. carpolithus Dolin, 1975 & Kazakhstan: Karabastau & $166.1-157.3(\mathrm{~J})$ \\
\hline & H. confusus Dolin, 1975 & Kazakhstan: Karabastau & $166.1-157.3(\mathrm{~J})$ \\
\hline & H. curtus Dolin, 1980 & Kazakhstan: Karabastau & $166.1-157.3(\mathrm{~J})$ \\
\hline & H. distinctus Dolin, 1975 & Kazakhstan: Karabastau & $164.7-155.7(\mathrm{~J})$ \\
\hline & H. dubius Dolin, 1975 & Kazakhstan: Karabastau & $166.1-157.3(\mathrm{~J})$ \\
\hline & H. gigas Dolin, 1980 & Kazakhstan: Karabastau & $166.1-157.3(\mathrm{~J})$ \\
\hline & H. imperspicuus Dolin, 1975 & Kazakhstan: Karabastau & $166.1-157.3(\mathrm{~J})$ \\
\hline & H. induratus Dolin, 1975 & Kazakhstan: Karabastau & $166.1-157.3(\mathrm{~J})$ \\
\hline & H. inventus Dolin, 1975 & Kazakhstan: Karabastau & $166.1-157.3(\mathrm{~J})$ \\
\hline
\end{tabular}


Table A1. Cont.

\begin{tabular}{|c|c|c|c|}
\hline Subfamily, Tribe, Genus & Species & Geographic Origin & Age (Ma), Period/Epoch \\
\hline & H. minutus Dolin, 1975 & Kazakhstan: Karabastau & $166.1-157.3(\mathrm{~J})$ \\
\hline & H. rasnitzyni Dolin, 1980 & Kazakhstan: Karabastau & $166.1-157.3(\mathrm{~J})$ \\
\hline & H. rohdendorfi Dolin, 1975 & Kazakhstan: Karabastau & $166.1-157.3(\mathrm{~J})$ \\
\hline \multirow[t]{3}{*}{ Lapidiconides Dolin, 1980} & L. brevis Dolin, 1980 & Kazakhstan: Karabastau & $166.1-157.3(\mathrm{~J})$ \\
\hline & L. excellens Dolin, 1980 & Kazakhstan: Karabastau & $166.1-157.3(\mathrm{~J})$ \\
\hline & L. innatus Dolin, 1980 & Kazakhstan: Karabastau & $166.1-157.3(\mathrm{~J})$ \\
\hline \multirow[t]{5}{*}{ Lapidostenus Dolin, 1980} & L. infossus Dolin, 1980 & Kazakhstan: Karabastau & $166.1-157.3(\mathrm{~J})$ \\
\hline & L. insignis Dolin, 1980 & Kazakhstan: Karabastau & $166.1-157.3(\mathrm{~J})$ \\
\hline & L. longicornis Dolin, 1980 & Kazakhstan: Karabastau & $166.1-157.3(\mathrm{~J})$ \\
\hline & L. scutellaris Dolin, 1980 & Kazakhstan: Karabastau & $166.1-157.3(\mathrm{~J})$ \\
\hline & L. tarbinskyi Dolin, 1980 & Kazakhstan: Karabastau & $166.1-157.3(\mathrm{~J})$ \\
\hline \multirow[t]{4}{*}{ Lithoptychus Dolin, 1980} & L. carinatissimus Dolin, 1980 & Kazakhstan: Karabastau & $166.1-157.3(\mathrm{~J})$ \\
\hline & L. handlirschi Dolin, 1980 & Kazakhstan: Karabastau & $166.1-157.3(\mathrm{~J})$ \\
\hline & L. incertus Dolin, 1980 & Kazakhstan: Karabastau & $166.1-157.3(\mathrm{~J})$ \\
\hline & L. minutus Dolin, 1980 & Kazakhstan: Karabastau & $166.1-157.3(\mathrm{~J})$ \\
\hline \multirow[t]{2}{*}{ Lithosomus Dolin, 1980} & L. erosus Dolin, 1980 & Kazakhstan: Karabastau & $166.1-157.3(\mathrm{~J})$ \\
\hline & L. longicollis Dolin, 1980 & Kazakhstan: Karabastau & $166.1-157.3(\mathrm{~J})$ \\
\hline Necrocoelus Dolin, 1980 & N. aselloides Dolin, 1980 & Kazakhstan: Karabastau & $166.1-157.3(\mathrm{~J})$ \\
\hline \multirow[t]{4}{*}{ Negastrioides Dolin, 1980} & N. globicollis Dolin, 1980 & Kazakhstan: Karabastau & $166.1-157.3(\mathrm{~J})$ \\
\hline & N. tenuicornis Dolin, 1980 & Kazakhstan: Karabastau & $166.1-157.3(\mathrm{~J})$ \\
\hline & N. tenuis Dolin, 1980 & Kazakhstan: Karabastau & $166.1-157.3(\mathrm{~J})$ \\
\hline & N. tscherepanovi Dolin, 1980 & Kazakhstan: Karabastau & $166.1-157.3(\mathrm{~J})$ \\
\hline \multirow[t]{3}{*}{ Parahypnomorphus Dolin, 1980} & P. jurassicus Dolin, 1980 & Kazakhstan: Karabastau & $166.1-157.3(\mathrm{~J})$ \\
\hline & P. longicornis Dolin, 1980 & Kazakhstan: Karabastau & $166.1-157.3(\mathrm{~J})$ \\
\hline & P. similis Dolin, 1980 & Kazakhstan: Karabastau & $166.1-157.3(\mathrm{~J})$ \\
\hline \multirow[t]{4}{*}{ Platyelater Dolin, 1980} & P. figeratus Dolin, 1980 & Kazakhstan: Karabastau & $166.1-157.3(\mathrm{~J})$ \\
\hline & P. quiescentus Dolin, 1980 & Kazakhstan: Karabastau & $166.1-157.3(\mathrm{~J})$ \\
\hline & P. reflexicollis Dolin, 1980 & Kazakhstan: Karabastau & $166.1-157.3(\mathrm{~J})$ \\
\hline & P. sukatschevae Dolin, 1980 & Kazakhstan: Karabastau & $166.1-157.3(\mathrm{~J})$ \\
\hline \multicolumn{4}{|l|}{ Pollostelaterini } \\
\hline Pollostelater Alekseev, 2011 & P. baissensis Alekseev, 2011 & Russia: Zaza & $125.0-113.0(\mathrm{C})$ \\
\hline \multicolumn{4}{|l|}{ Protagrypnini } \\
\hline \multirow[t]{3}{*}{ Acheonus Dolin, 1980} & A. abbreviatus Dolin, 1980 & Kazakhstan: Karabastau & $166.1-157.3(\mathrm{~J})$ \\
\hline & A. gracilis Dolin, 1980 & Kazakhstan: Karabastau & $166.1-157.3(\mathrm{~J})$ \\
\hline & A. minutissimus Dolin, 1980 & Kazakhstan: Karabastau & $166.1-157.3(\mathrm{~J})$ \\
\hline $\begin{array}{l}\text { Clavelater Dong and Huang, } \\
2011\end{array}$ & $\begin{array}{c}\text { C. ningchengensis Dong and } \\
\text { Huang, } 2011\end{array}$ & China: Jiulongshan & $166.1-157.3(\mathrm{~J})$ \\
\hline $\begin{array}{l}\text { Koreagrypnus Sohn and Nam, } \\
2019\end{array}$ & K. jinju Sohn and Nam, 2019 & South Korea: Jinju & $113.0-100.5(\mathrm{C})$ \\
\hline Lithocoelus Dolin, 1975 & L. detrusus Dolin, 1975 & Kazakhstan: Karabastau & $166.1-157.3(\mathrm{~J})$ \\
\hline
\end{tabular}


Table A1. Cont.

\begin{tabular}{|c|c|c|c|}
\hline Subfamily, Tribe, Genus & Species & Geographic Origin & Age (Ma), Period/Epoch \\
\hline & L. karatavicus Dolin, 1975 & Kazakhstan: Karabastau & $166.1-157.3(\mathrm{~J})$ \\
\hline \multirow[t]{7}{*}{ Lithomerus Dolin, 1980} & L. brachycollis Dolin, 1980 & Kazakhstan: Karabastau & $166.1-157.3(\mathrm{~J})$ \\
\hline & L. brevicollis Dolin, 1980 & Kazakhstan: Karabastau & $166.1-157.3(\mathrm{~J})$ \\
\hline & L. buyssoni Dolin and Nel, 2002 & China: Yixian & $125.45-122.46(\mathrm{C})$ \\
\hline & L. cockerelli Dolin, 1980 & Kazakhstan: Karabastau & $166.1-157.3(\mathrm{~J})$ \\
\hline & L. contiguus Dolin, 1980 & Kazakhstan: Karabastau & $166.1-157.3(\mathrm{~J})$ \\
\hline & L. longulus Dolin, 1980 & Kazakhstan: Karabastau & $166.1-157.3(\mathrm{~J})$ \\
\hline & L. wunda Martin, 2010 & $\begin{array}{c}\text { Australia: Cattamarra Coal } \\
\text { Measures }\end{array}$ & $182.7-174.1(\mathrm{~J})$ \\
\hline $\begin{array}{l}\text { Megalithomerus Sohn and } \\
\text { Nam, } 2019\end{array}$ & $\begin{array}{l}\text { M. magohalmii Sohn and Nam, } \\
2019\end{array}$ & South Korea: Jinju & $113.0-100.5(\mathrm{C})$ \\
\hline Micragrypnites Dolin, 1973 & M. issykiensis Dolin, 1973 & Kyrgyzstan: Dzhil & 201.3-190.8 (J) \\
\hline Paragrypnites Dolin, 1980 & P. jagemanni Dolin, 1980 & Kazakhstan: Karabastau & $166.1-157.3(\mathrm{~J})$ \\
\hline $\begin{array}{l}\text { Paraprotagrypnus Chang et al., } \\
2009\end{array}$ & P. superbus Chang et al., 2009 & China: Jiulongshan & $166.1-157.3(\mathrm{~J})$ \\
\hline \multirow[t]{2}{*}{ Protagrypnus Dolin, 1973} & P. exoletus Dolin, 1973 & Kyrgyzstan: Dzhil & 201.3-190.8 (J) \\
\hline & P. robustus Chang et al., 2009 & China: Jiulongshan & $166.1-157.3(\mathrm{~J})$ \\
\hline $\begin{array}{l}\text { Sinolithomerus Dong and } \\
\text { Huang, } 2009\end{array}$ & S. dolini Dong and Huang, 2009 & China: Haifanggou & $166.1-157.3(\mathrm{~J})$ \\
\hline \multicolumn{4}{|l|}{ Elateridae incertae sedis } \\
\hline Adocetus Scudder, 1900 & A. buprestoides Scudder, 1900 & USA: Green River & $55.8-50.3(\mathrm{E})$ \\
\hline Artinama Lin, 1986 & A. qinghuoensis Lin, 1986 & China: Zaoshang & $199.3-190.8(\mathrm{~J})$ \\
\hline $\begin{array}{l}\text { Bilineariselater Chang and Ren, } \\
\qquad 2008\end{array}$ & B. foveatus Chang and Ren, 2008 & China: Yixian & $125.45-122.46(\mathrm{C})$ \\
\hline Cretoelaterium Alekseev, 2008 & C. kazanovense Alekseev, 2008 & Russia: Mirsanovo & $129.4-125.0(\mathrm{C})$ \\
\hline Cryptagriotes Wickham, 1916 & C. minusculus Wickham, 1916 & USA: Florissant & $37.2-33.9(\mathrm{E})$ \\
\hline \multirow[t]{8}{*}{$\begin{array}{l}\text { Cryptocoelus Dolin and Nel, } \\
2002\end{array}$} & C. baissensis Alekseev, 2011 & Russia: Zaza & 125.0-113.0 (C) \\
\hline & C. buffoni Dolin and Nel, 2002 & China: Yixian & $125.45-122.46(\mathrm{C})$ \\
\hline & C. dolini Alekseev, 2011 & Russia: Zaza & $125.0-113.0(\mathrm{C})$ \\
\hline & C. gianteus Chang et al., 2007 & China: Yixian & $125.45-122.46(\mathrm{C})$ \\
\hline & C. lukashevichae Alekseev, 2011 & Russia: Zaza & $125.0-113.0(\mathrm{C})$ \\
\hline & C. major Dolin and Nel, 2002 & China: Yixian & $125.45-122.46(\mathrm{C})$ \\
\hline & C. shcherbakovi Alekseev, 2011 & Russia: Zaza & $125.0-113.0(\mathrm{C})$ \\
\hline & C. sinitshenkovae Alekseev, 2011 & Russia: Zaza & $125.0-113.0(\mathrm{C})$ \\
\hline $\begin{array}{l}\text { Curtelater Chang and Ren, } \\
2008\end{array}$ & C. wui Chang and Ren, 2008 & China: Yixian & $125.45-122.46(\mathrm{C})$ \\
\hline \multirow[t]{3}{*}{ Elateridium Tillyard, 1918} & E. subulatum (Dunstan, 1923) & Australia: Blackstone & $228.0-208.5(\mathrm{~T})$ \\
\hline & E. transversum (Dunstan, 1923) & Australia: Blackstone & $228.0-208.5(\mathrm{~T})$ \\
\hline & E. wianamattense (Tillyard, 1916) & Australia: Ashfield Shales & $247.2-242.0(\mathrm{~T})$ \\
\hline Elaterites Heer, 1847 & E. amissus Heer, 1847 & $\begin{array}{l}\text { Switzerland: Greith coal } \\
\text { mine }\end{array}$ & $28.4-23.03(\mathrm{O})$ \\
\hline
\end{tabular}


Table A1. Cont.

\begin{tabular}{|c|c|c|c|}
\hline Subfamily, Tribe, Genus & Species & Geographic Origin & Age (Ma), Period/Epoch \\
\hline & E. bruchi Cockerell, 1926 & Argentina: Margas Verdes & $66.0-56.0(\mathrm{P})$ \\
\hline & E. dicrepidioides Deichmüller, 1881 & Czech Republic: Kučlín & $37.2-33.9(\mathrm{E})$ \\
\hline & E. laconoides Cockerell, 1920 & United Kingdom: Poole & $47.8-41.3(\mathrm{E})$ \\
\hline & E. lavateri Heer, 1847 & $\begin{array}{l}\text { Germany: Upper } \\
\text { Freshwater-Molasse }\end{array}$ & $12.7-11.608(\mathrm{M})$ \\
\hline & E. longus Haupt, 1956 & Germany: Geiseltal & $47.8-41.3(\mathrm{E})$ \\
\hline & E. microstictus Cockerell, 1926 & Argentina: Margas Verdes & $66.0-56.0(\mathrm{P})$ \\
\hline & E. murchisoni (Giebel, 1856) & United Kingdom: Poole & $47.8-41.3(\mathrm{E})$ \\
\hline & E. obsoletus Heer, 1847 & $\begin{array}{l}\text { Germany: Upper } \\
\text { Freshwater-Molasse }\end{array}$ & $12.7-11.608(\mathrm{M})$ \\
\hline & E. palaeophilus Cockerell, 1920 & United Kingdom: Peckham & $56.0-47.8(\mathrm{E})$ \\
\hline & E. perditulus Cockerell, 1920 & United Kingdom: Poole & $47.8-41.3(\mathrm{E})$ \\
\hline & E. sculptilis Cockerell, 1920 & United Kingdom: Poole & $47.8-41.3(\mathrm{E})$ \\
\hline \multirow[t]{2}{*}{ Elaterium Westwood, 1854} & E. bipunctatum Dunstan, 1923 & Australia: Blackstone & $228.0-208.5(\mathrm{~T})$ \\
\hline & E. pronaeus Westwood, 1854 & United Kingdom: Lulworth & $145.0-140.2(\mathrm{C})$ \\
\hline Gripecolous Lin, 1986 & G. enallus Lin, 1986 & China: Shiti & $170.3-168.3(\mathrm{~J})$ \\
\hline Ludiophanes Wickham, 1916 & L. haydeni Wickham, 1916 & USA: Florissant & $37.2-33.9(\mathrm{E})$ \\
\hline Mercata Lin, 1986 & M. festira Lin, 1986 & China: Shiti & $170.3-168.3(\mathrm{~J})$ \\
\hline Mionelater Becker, 1963 & M. planatus Becker, 1963 & Mexico: Chiapas amber & 23.03-15.97 (M) \\
\hline Ovivagina Zhang, 1997 & O. longa Zhang, 1997 & China: Badaowan & 201.3-190.8 (J) \\
\hline \multirow[t]{2}{*}{$\begin{array}{l}\text { Paralithomerus Chang et al., } \\
2008\end{array}$} & P. exquisitus Chang et al., 2008 & China: Yixian & $125.45-122.46(\mathrm{C})$ \\
\hline & P. parallelus Chang et al., 2008 & China: Yixian & $125.45-122.46(\mathrm{C})$ \\
\hline \multirow[t]{2}{*}{ Protocardiophorus Dolin, 1976} & P. ancestralis Dolin, 1976 & Kazakhstan: Karabastau & $166.1-157.3(\mathrm{~J})$ \\
\hline & P. jurassicus Dolin, 1980 & Kazakhstan: Karabastau & $166.1-157.3(\mathrm{~J})$ \\
\hline \multirow[t]{5}{*}{$\begin{array}{l}\text { Pseudocardiophorites Dolin, } \\
1976\end{array}$} & P. angustatus Dolin, 1980 & Kazakhstan: Karabastau & $166.1-157.3(\mathrm{~J})$ \\
\hline & P. fragilis Dolin, 1976 & Kazakhstan: Karabastau & $166.1-157.3(\mathrm{~J})$ \\
\hline & P. hayeki Dolin, 1976 & Kazakhstan: Karabastau & $166.1-157.3(\mathrm{~J})$ \\
\hline & P. infractus Dolin, 1976 & Kazakhstan: Karabastau & $166.1-157.3(\mathrm{~J})$ \\
\hline & P. quadricollis Dolin, 1976 & Kazakhstan: Karabastau & $166.1-157.3(\mathrm{~J})$ \\
\hline Silicernius Heyden, 1859 & S. spectabilis Heyden, 1859 & Germany: Rott & $28.4-23.03(\mathrm{O})$ \\
\hline Sinoelaterium Ping, 1928 & S. melanocolor Ping, 1928 & China: Yixian & $125.45-122.46(\mathrm{C})$ \\
\hline $\begin{array}{c}\text { Tetraraphes } \\
\text { Iablokoff-Khnzorian, } 1961\end{array}$ & $\begin{array}{l}\text { T. ebersini Iablokoff-Khnzorian, } \\
1961\end{array}$ & Europe: Baltic amber & $38.0-33.9(\mathrm{E})$ \\
\hline Turonelater Alekseev, 2011 & T. giganteus Alekseev, 2011 & Kazakhstan: Kzyl-Zhar & $93.9-89.8(\mathrm{C})$ \\
\hline Genus incertae sedis & $\begin{array}{c}\text { Acmaeodera burmitina Cockerell, } \\
1917\end{array}$ & Myanmar: Burmese amber & $99.6-93.5(C)$ \\
\hline
\end{tabular}

\section{References}

1. Costa, C.; Lawrence, J.F.; Rosa, S.P. Elateridae Leach, 1815. In Handbook of Zoology, Arthropoda: Insecta; Coleoptera, Beetles; Volume 2: Morphology and Systematics (Elateroidea, Bostrichiformia, Cucujiformia partim); Leschen, R.A.B., Beutel, R.G., Lawrence, J.F., Eds.; Walter de Gruyter GmbH \& Co. KG: Berlin, Germany, 2010; pp. 75-103.

2. Calder, A.A.; Lawrence, J.F.; Trueman, J.W.H. Austrelater, gen. nov. (Coleoptera: Elateridae), with a description of the larva and comments on elaterid relationships. Invertebr. Taxon. 1993, 7, 1349-1394. [CrossRef] 
3. Sagegami-Oba, R.; Oba, Y.; Ôhira, H. Phylogenetic relationships of click beetles (Coleoptera: Elateridae) inferred from 28S ribosomal DNA: Insights into the evolution of bioluminescence in Elateridae. Mol. Phylogenet. Evol. 2007, 42, 410-421. [CrossRef] [PubMed]

4. Douglas, H. Phylogenetic relationships of Elateridae inferred from adult morphology, with special reference to the position of Cardiophorinae. Zootaxa 2011, 2900, 1-45. [CrossRef]

5. Kundrata, R.; Gunter, N.L.; Douglas, H.; Bocak, L. Next step toward a molecular phylogeny of click-beetles (Coleoptera: Elateridae): Redefinition of Pityobiinae, with a description of a new subfamily, Parablacinae, from the Australasian Region. Austral Entomol. 2016, 55, 291-302. [CrossRef]

6. Kundrata, R.; Gunter, N.L.; Janosikova, D.; Bocak, L. Molecular evidence for the subfamilial status of Tetralobinae (Coleoptera: Elateridae), with comments on parallel evolution of some phenotypic characters. Arthropod Syst. Phylogeny 2018, 76, 137-145.

7. Kusy, D.; Motyka, M.; Bocek, M.; Vogler, A.P.; Bocak, L. Genome sequences identify three families of Coleoptera as morphologically derived click beetles (Elateridae). Sci. Rep. 2018, 8, 17084. [CrossRef]

8. Bi, W.-X.; He, J.-W.; Chen, C.-C.; Kundrata, R.; Li, X.-Y. Sinopyrophorinae, a new subfamily of Elateridae (Coleoptera, Elateroidea), with the first record of a luminous click beetle in Asia and evidence for multiple origins of bioluminescence in Elateridae. ZooKeys 2019, 864, 79-97. [CrossRef] [PubMed]

9. Kundrata, R.; Prosvirov, A.S.; Vondracek, D.; Sormova, E. Congruence between molecular data and morphology: Phylogenetic position of Senodoniini (Coleoptera: Elateridae). Insects 2019, 10, 231. [CrossRef]

10. Kusy, D.; He, J.-W.; Bybee, S.M.; Motyka, M.; Bi, W.-X.; Podsiadlowski, L.; Li, X.-Y.; Bocak, L. Phylogenomic relationships of bioluminescent elateroids define the 'lampyroid' clade with clicking Sinopyrophoridae as its earliest member. Syst. Entomol. 2021, 46, 111-123. [CrossRef]

11. Kusy, D.; Motyka, M.; Bocak, L. Click beetle mitogenomics with the definition of a new subfamily Hapatesinae from Australasia (Coleoptera: Elateridae). Insects 2021, 12, 17. [CrossRef] [PubMed]

12. Kundrata, R.; Packova, G.; Hoffmannova, J. Fossil genera in Elateridae (Insecta, Coleoptera): A Triassic origin and Jurassic diversification. Insects 2020, 11, 394. [CrossRef]

13. Brodie, P.B. A History of the Fossil Insects in the Secondary Rocks of England Accompanied by a Particular Account of the Strata in Which they Occur, and of the Circumstances Connected with Their Preservation; John Van Voorst: London, UK, 1845; pp. 1-130.

14. Heer, O. Die Insektenfauna der Tertiärgebilde von Oeningen und von Radoboj in Croatien; Erster Theil: Käfer; Wilhelm Engelmann: Leipzig, Germany, 1847; pp. 1-229.

15. Westwood, J.O. Contributions to fossil entomology. Quart. J. Geol. Soc. Lond. 1854, 10, 378-396. [CrossRef]

16. Giebel, C.G. Fauna der Vorwelt Mit Steter Berücksichtigung der Lebenden Thiere. Monographisch Dargestellt. Zweiter Band: Gliederthiere. Erste Abtheilung. Insecten und Spinnen; Brockhaus: Leipzig, Germany, 1856; pp. 1-511.

17. Heer, O. Die Urwelt der Schweiz; Friedrich Schulthess: Zürich, Switzerland, 1865; pp. 1-622.

18. Heer, O. Die Miocene flora und fauna Spitzbergens. Kongl. Sven. Vetenskapsakad. Handl. 1870, 8, 1-98.

19. Scudder, S.H. Fossil Coleoptera from the Rocky Mountain Tertiaries. Bull. U. S. Geol. Geogr. Surv. Terr. 1876, 2, 77-87.

20. Scudder, S.H. The Fossil Insects Collected in 1877, by Mr. G.M. Dawson, in the Interior of British Columbia. Geological Survey of Canada, Report of Progress for 1877-1878; Dawson Brothers: Montreal, QC, Canada, 1879; pp. 175-185.

21. Deichmüller, J.V. Fossile Insecten aus dem Diatomeenschiefer von Kutschlin bei Bilin, Böhmen. Nova Acta Acad. Caes. Leop. Carol. German. Nat. Cur. 1881, 42, 293-330.

22. Scudder, S.H. Classe Insecta. Insecta; In Handbuch der Palaeontologie; I Abtheilung. Palaeozoologie 2; Zittel, K., Ed.; Oldenbourg: München, Germany; Leipzig, Germany, 1885; pp. 747-831.

23. Scudder, S.H. Systematic Review of our Present Knowledge of Fossil Insects including Myriapods and Arachnids; Government Printing Office: Washington, DC, USA, 1886; pp. 1-128.

24. Scudder, S.H. Index to the Known Fossil Insects of the World including Myriapods and Arachnids; US Government Printing Office: Washington, DC, USA, 1891; Volume 71, pp. 1-744.

25. Lomnicki, J.L.M. Elater wisniowskii, nov. sp. Spraw. Kom. Fizyogr. Mater. Fizyogr. Kraj. 1902, 36, 11-12.

26. Handlirsch, A. Die Fossilen Insekten und Die Phylogenie der Rezenten Formen, Parts I-IV. Ein Handbuch fur Palaontologen und Zoologen; Wilhelm Engelmann: Leipzig, Germany, 1906; pp. 1-640.

27. Wickham, H.F. New fossil Elateridae from Florissant. Am. J. Sci. 1908, 26, 76-78. [CrossRef]

28. Wickham, H.F. The fossil Elateridae of Florissant. Bull. Mus. Comp. Zool. 1916, 60, 493-527.

29. Wickham, H.F. Catalogue of the North American Coleoptera described as fossils. In Catalogue of the Coleoptera of America North of Mexico; Leng, C.W., Ed.; Sherman: Mt. Vernon, NY, USA, 1920; pp. 349-365.

30. Klebs, R. Über Bernsteineinschlüsse im allgemeinen und die Coleopteren meiner Bernsteinsammlung. Schrift. Physik. Ökonom. Gesellsch. Königsberg 1910, 51, 217-242.

31. Cockerell, T.D.A. British fossil insects. Proc. U. S. Nat. Mus. 1916, 49, 469-499. [CrossRef]

32. Cockerell, T.D.A. Fossil insects. Ann. Entomol. Soc. Am. 1917, 10, 1-18. [CrossRef]

33. Cockerell, T.D.A. Insects in Burmese amber. Ann. Entomol. Soc. Am. 1917, 10, 323-329. [CrossRef]

34. Cockerell, T.D.A. Fossil Arthropods in the British Museum, III. Ann. Mag. Nat. Hist. 1920, 5, 455-463. [CrossRef]

35. Cockerell, T.D.A. Some Tertiary fossil insects. Ann. Mag. Nat. Hist. 1926, 18, 313-324. [CrossRef]

36. Cockerell, T.D.A.; LeVeque, N. The antiquity of insect structures. Am. Nat. 1931, 65, 351-359. [CrossRef] 
37. Tillyard, R.J. Mesozoic and Tertiary Insects of Queensland and New South Wales. Descriptions of the fossil Insects. Qld. Geol. Surv. Pub. 1916, 253, 11-70.

38. Tillyard, R.J. Permian and Triassic insects from New South Wales, in the collection of Mr. John Mitchell. Proc. Linn. Soc. N. S. W. 1918, 42, 720-756.

39. Dunstan, B. Mesozoic Insects of Queensland Part I.-Introduction and Coleoptera. Qld. Geol. Surv. Pub. 1923, $273,1-89$.

40. Ping, C. Study of the Cretaceous fossil insects of China. Palaeontol. Sinica Ser. B 1928, 13, 1-56.

41. Wickham, H.F. Coleoptera from the lower Eocene (Wilcox) clays. J. Wash. Acad. Sci. 1929, 19, 148-150.

42. Theobald, N. Les insectes fossiles des terrains oligocènes de France. Bull. Mens. Mem. Soc. Sci. Nancy 1937, 1, 1-473.

43. Piton, L. Paléontologie du gisement éocène de Menat (Puy-de-Dôme) (flore et faune). Mém. Soc. Hist. Nat. Auvergne 1940, 1 , 1-303.

44. Haupt, H. Die Käfer (Coleoptera) aus der eozänen Braunkohle des Geiseltales. Geologica 1950, 6, 1-168.

45. Gardiner, B.G. New Rhaetic and Liassic beetles. Palaeontology 1961, 4, 87-89.

46. Becker, E.C. Three new fossil elaterids from the amber of Chiapas, Mexico, including a new genus (Coleoptera). J. Paleontol. 1963, $37,125-128$.

47. Iablokoff-Khnzorian, S.M. Novye zhestkokrylye semeystva Elateridae iz baltiyskogo yantayra. Paleontol. Zh. 1961, $1961,84-97$.

48. Larsson, S.G. Baltic amber-A palaeobiological study. Entomonograph 1978, 1, 1-192.

49. Spahr, U. Systematischer Katalog der Bernstein- und Kopal-Käfer (Coleoptera). Systematic catalogue of Coleoptera in amber and copal. Stuttg. Beitr. Naturkd. Ser. B 1981, 80, 1-107.

50. Dolin, V.G. Iskopaemye formy Zhukov-Shchelkunov (Elateridae, Coleoptera) iz Nizhney Yury Sredney Azii. In Fauna i Biologiya Nasekomykh Moldavii, 3rd ed.; Yaroshenko, M., Ed.; Shtiintsa: Kishinev, Moldova, 1973; pp. 71-82.

51. Dolin, V.G. K sistematike mezozoiskikh zhukov-schchelkunov (Coleoptera, Elateridae). Paleontol. Zhurnal 1975, 9, 51-62.

52. Dolin, V.G. Fossil click beetles (Coleoptera, Elateridae) of the subfamilies Negastriinae and Cardophorinae from Upper Jurassic of Karatau. Vestn. Zool. 1976, 1976, 68-75.

53. Dolin, V.G. Click beetles (Coleoptera, Elateridae) from the Upper Jurassic of Karatau. In Fossil Insects of the Mesozoic; Dolin, V.G., Panfilov, D.V., Ponomarenko, A.G., Pritykina, D.N., Eds.; Naukova Dumka: Kiev, Ukraine, 1980; pp. 17-81.

54. Crowson, R.A. The Biology of the Coleoptera; Academic Press: London, UK, 1981; pp. 1-802.

55. Lin, Q.B. Early Mesozoic fossil insects from South China. Palaeontol. Sin. Ser. B 1986, 170, 1-112.

56. Zhang, H.C. A new genus of Elateridae (Insecta, Coleoptera) from Lower-Middle Jurassic of Junggar Basin, Xinjiang, China. Acta Micropal. Sin. 1997, 14, 71-77.

57. Hong, Y.C. Insecta. In Palaeontological Atlas of North China. Part II: Mesozoic; Yang, Y., Ed.; Geological Publishing House: Beijing, China, 1984; pp. 128-185.

58. Zhang, J. Fossil Insects from Shanwang, Shandong, China; Shandong Science and Technology Publishing House: Jinan, China, 1989; pp. $1-459$.

59. Zhang, J.; Sun, B.; Zhang, X. Miocene Insects and Spiders from Shanwang, Shandong; Science Press: Beijing, China, $1994 ;$ pp. 1-298.

60. Whalley, P.E.S. The systematics and palaeogeography of the Lower Jurassic insects of Dorset, England. Bull. Br. Mus. Nat. Hist. Geol. 1985, 39, 107-189.

61. Zaragoza Caballero, S. Descripción de una especie nueva fósil de Glyphonyx Candèze (Coleoptera: Elateridae, Adrastinae) del ámbar de Simojovel, Chiapas, México. In Anales del Instituto de Biología, Universidad Nacional Autónoma de México, 3rd ed.; Serie zoología; Universidad Nacional Autónoma de México, Instituto de Biología: Mexico City, Mexico, 1990; Volume 61, pp. 147-151.

62. Tröster, G. Eine neue Gattung der Elateridae (Insecta: Coleoptera) Macropunctum gen. n. aus der Messel-Formation des unteren Mittel-Eozän der Fundstätte Messel. Cour. Forschungsinst. Senck. 1991, 139, 99-117.

63. Tröster, G. Zur Kenntnis der Gattung Macropunctum (Elateridae, Pyrophorinae, Agrypnini) aus der mitteleozänen Fossillagerstätte "Eckfelder Maar" (Eifel) mit der Beschreibung einer neuen Art Macropunctum eckfeldi n. sp. Mainz. Naturwiss. Arch. 1992, 30, 111-118.

64. Tröster, G. Fossile Schnellkäfer der Gattung Lanelater Arnett 1952 (Coleoptera, Pyrophorinae, Agrypnini) aus dem Eozän der Grube Messel bei Darmstadt. Senck. Leth. 1993, 73, 49-60.

65. Tröster, G. Fossile Elateridae (Insecta: Coleoptera) aus dem unteren Mitteleozän (Lutetium) der Grube Messel bei Darmstadt. Cour. Forschungsinst. Senck. 1994, 170, 11-64.

66. Tröster, G. Neue Arten der Gattung Macropunctum (Insecta, Coleoptera, Elateridae) aus der Olschieferfazies der mitteleozänen Messelformation der Grube Messel bei Darmstadt. Palaontol. Zhurnal 1994, 68, 145-162. [CrossRef]

67. Tröster, G. An unusual new fossil click-beetle (Coleoptera: Elateridae) from the Middle Eocene of the Grube Messel (Germany). N. Jahrb. Geol. Palaeont. Mon. 1999, 1999, 11-20. [CrossRef]

68. Carpenter, F.M. Volume 4: Superclass Hexapoda. In Treatise on Invertebrate Paleontology; Part R, Arthropoda 4; Kaesler, R.D., Ed.; Geological Society of America: Boulder, CO, USA; University of Kansas: Lawrence, KS, USA, 1992; pp. $279-655$.

69. Wappler, T. Die Insekten aus dem Mittel-Eozän des Eckfelder Maares, Vulkaneifel. Mainzer Naturwiss. Arch. 2003, $27,1-234$.

70. Kirejtshuk, A.G.; Ponomarenko, A.G.; Kurochkin, A.S.; Alekseev, A.V.; Gratshev, V.G.; Solodovnikov, A.V.; Krell, F.-T.; Soriano, C. The beetle (Coleoptera) fauna of the Insect Limestone (late Eocene), Isle of Wight, southern England. Earth Environ. Sci. Trans. R. Soc. Edinb. 2019, 110, 405-492. [CrossRef] 
71. Martins-Neto, R.G.; Gallego, O.F.; Mancuso, A.C. The Triassic insect fauna from Argentina. Coleoptera from Los Rastros Formation (Bermejo Basin), La Rioja Province. Ameghiniana 2006, 43, 591-609.

72. Martins-Neto, R.G.; Gallego, O.F. The Triassic insect fauna from Argentina. Blattoptera and Coleoptera from the Ischichuca Formation (Bermejo Basin), La Rioja Province. Ameghiniana 2009, 46, 361-372.

73. Martin, S.K. Early Jurassic coleopterans from the Mintaja insect locality, Western Australia. Acta Geol. Sin. 2010, 84, 925-953. [CrossRef]

74. Alekseev, A.V. New jewel beetles (Coleoptera: Buprestidae) and similar beetles from the Cretaceous and Early Paleogene of Asia. Paleontol. J. 2008, 42, 53-59.

75. Alekseev, A.V. New click beetles (Coleoptera: Elateridae) from the Cretaceous of Russia and Kazakhstan. Paleontol. J. 2011, 45, 423-431.

76. Sohn, J.C.; Nam, G.S.; Choi, S.W.; Ren, D. New fossils of Elateridae (Insecta, Coleoptera) from Early Cretaceous Jinju Formation (South Korea) with their implications to evolutionary diversity of extinct Protagrypninae. PLoS ONE 2019, 14, e0225502. [CrossRef]

77. Chang, H.L.; Kirejtshuk, A.G.; Ren, D. On taxonomy and distribution of fossil Cerophytidae (Coleoptera: Elateriformia) with description of a new Mesozoic species of Necromera Martynov, 1926. Ann. Soc. Entomol. Fr. 2011, 47, 33-44. [CrossRef]

78. Chang, H.L.; Kirejtshuk, A.G.; Ren, D. First fossil click beetles from the Middle Jurassic of Inner Mongolia, China (Coleoptera: Elateridae). Ann. Zool. 2009, 59, 7-14. [CrossRef]

79. Chang, H.L.; Zhao, Y.Y.; Ren, D. New fossil elaterids (Insect: Coleoptera: Polyphaga: Elateridae) from the Middle Jurassic of Inner Mongolia, China. Prog. Nat. Sci. 2009, 19, 1433-1437. [CrossRef]

80. Dong, F.B.; Huang, D.Y. A new click beetle (Coleoptera: Elateridae) from Middle Jurassic Haifanggou Formation of western Liaoning, China. Acta Pal. Sin. 2009, 48, 102-108.

81. Dong, F.B.; Huang, D.Y. A new elaterid from the Middle Jurassic Daohugou biota (Coleoptera: Elateridae: Protagrypninae). Acta Geol. Sin. 2011, 85, 1224-1230.

82. Dolin, V.G.; Nel, A. Trois nouveaux Elateridae fossiles du Mésozoïque supérieur de Chine (Coleoptera). Bull. Soc. Entomol. Fr. 2002, 107, 341-346.

83. Chang, H.L.; Ren, D.; Shih, C.K. New fossil elaterid (Coleoptera: Polyphaga: Elateridae) from Yixian Formation of western Liaoning, China. Prog. Nat. Sci. 2007, 17, 1244-1249.

84. Chang, H.L.; Ren, D. New fossil beetles of the family Elateridae from the Jehol Biota of China (Coleoptera: Polyphaga). Acta Geol. Sin. 2008, 82, 236-243.

85. Chang, H.L.; Zhang, F.; Ren, D. A new genus and two new species of fossil Elaterids from the Yixian Formation of western Liaoning, China (Coleoptera: Elateridae). Zootaxa 2008, 1785, 54-62. [CrossRef]

86. Chang, H.L.; Kirejtshuk, A.G.; Ren, D. New fossil elaterids (Coleoptera: Polyphaga: Elateridae) from the Jehol biota in China. Ann. Entomol. Soc. Am. 2010, 103, 866-874. [CrossRef]

87. Kirejtshuk, A.G.; Ponomarenko, A.G.; Prokin, A.A.; Chang, H.; Nikolajev, G.V.; Ren, D. Current knowledge on Mesozoic Coleoptera from Daohugou and Liaoning (Northeast China). Acta Geol. Sin. 2010, 84, 783-792. [CrossRef]

88. Ponomarenko, A.G.; Yan, E.V.; Wang, B.; Zhang, H.C. Revision of some early Mesozoic beetles from China. Acta Palaeontol. Sin. 2012, 51, 475-490.

89. Yu, Y.; Liu, Z.H.; Shih, C.H.; Ren, D. Coleoptera-Beetles. In Rhythms of Insect Evolution: Evidence from the Jurassic and Cretaceous in Northern China, 1st ed.; Ren, D., Shih, C.H., Gao, T., Yao, Y.U., Wang, Y., Eds.; John Wiley \& Sons, Ltd.: Hoboken, NJ, USA, 2019; pp. 337-428.

90. Solórzano Kraemer, M.M. Systematic, palaeoecology, and palaeobiogeography of the insect fauna from Mexican amber. Palaeontogr. Abt. A 2007, 282, 1-133. [CrossRef]

91. Schimmel, R. Die Megapenthini-Arten Süd- und Südostasiens. Vierter Teil: Phylogenese, Zoogeographie und Katalog (Coleoptera: Elateridae). Pollichia Buch 2005, 48, 1-411.

92. Alekseev, V.I. The beetles (Insecta: Coleoptera) of Baltic amber: The checklist of described species and preliminary analysis of biodiversity. Zool. Ecol. 2013, 23, 5-12. [CrossRef]

93. Kirejtshuk, A.G.; Kovalev, A.V. First fossil representative of the family Omalisidae (Coleoptera, Elateroidea sensu lato) from the Baltic amber. Paleontol. J. 2015, 49, 1413-1416. [CrossRef]

94. Kundrata, R.; Bukejs, A.; Prosvirov, A.S.; Hoffmannova, J. X-ray micro-computed tomography reveals a unique morphology in a new click-beetle (Coleoptera, Elateridae) from the Eocene Baltic amber. Sci. Rep. 2020, 10, 20158. [CrossRef]

95. Kirejtshuk, A.G.; Azar, D. Current knowledge of Coleoptera (Insecta) from the Lower Cretaceous Lebanese amber and taxonomical notes for some Mesozoic groups. Terr. Arthropod Rev. 2013, 6, 103-143. [CrossRef]

96. Otto, R.L. Descriptions of two new elateroid beetles (Coleoptera: Eucnemidae, Elateridae) from Burmese amber. Insecta Mundi 2019, 702, 1-6.

97. Kirejtshuk, A.G.; Ponomarenko, A.G. Taxonomical List of Fossil Beetles of the Suborder Scarabaeina (Part 2). [Last Update: July 2015]. Available online: https://www.zin.ru/Animalia/Coleoptera/eng/paleosy1.htm (accessed on 10 May 2020).

98. Oberprieler, R.G.; Ashman, L.G.; Frese, M.; Ślipiński, A. The first elateroid beetles Coleoptera: Polyphaga: Elateroidea) from the Upper Jurassic of Australia. Zootaxa 2016, 4147, 177-191. [CrossRef] [PubMed]

99. Muona, J.; Chang, H.; Ren, D. The clicking Elateroidea from Chinese Mesozoic deposits (Insecta, Coleoptera). Insects 2020, 11, 875. [CrossRef] [PubMed] 
100. Solórzano Kraemer, M.M.; Delclòs, X.; Engel, M.S.; Peñalver, E. A revised definition for copal and its significance for palaeontological and Anthropocene biodiversity-loss studies. Sci. Rep. 2020, 10, 19904. [CrossRef]

101. Gistel, J.N.F.X. Kerfe in Copal eingeschlossen. Isis Oken 1831, 3, 247-248.

102. Hope, F.W. Observations on succinic insects. Part the second. Gums and resins. Trans. Entomol. Soc. Lond. 1837, 2, 46-57. [CrossRef]

103. Quedenfeldt, G. Copal-Insecten aus Africa. Berl. Entomol. Z. 1885, 29, 363-365. [CrossRef]

104. Meunier, F. Nouvelles recherches sur quelques Diptères et Hyménoptères du copal fossile "dit de Zanzibar". Rev. Sci. Bourbon Cent. Fr. 1905, 18, 204-215.

105. Hawkeswood, T.J.; Makhan, D.; Turner, J.R. Cardiophorus jacquelinae sp. nov., a new click beetle (Coleoptera: Elateridae, Cardiophorinae) from Madagascan copal amber. G. Ital. Entomol. 2009, 12, 189-197.

106. Johnson, P.J. Elateridae Leach 1815. In American Beetles, Vol. 2, Polyphaga: Scarabaeoidea through Curculionoidea; Arnett, R.H., Thomas, M.C., Skelley, P.E., Frank, J.H., Eds.; CRC Press: Boca Raton, FL, USA, 2002; pp. 160-173.

107. Cate, P.C. Elateridae Leach, 1815 (-Cebrioninae, Lissominae, Subprotelaterinae). In Catalogue of Palaearctic Coleoptera; Löbl, I., Smetana, A., Eds.; Apollo Books: Stenstrup, Denmark, 2007; Volume 4, pp. 89-209.

108. Douglas, H. World reclassification of the Cardiophorinae (Coleoptera, Elateridae), based on phylogenetic analyses of morphological characters. ZooKeys 2017, 655, 1-130. [CrossRef] [PubMed]

109. Kundrata, R.; Kubaczkova, M.; Prosvirov, A.S.; Douglas, H.B.; Fojtikova, A.; Costa, C.; Bousquet, Y.; Alonso-Zarazaga, M.A.; Bouchard, P. World catalogue of the genus-group names in Elateridae (Insecta, Coleoptera). Part I: Agrypninae, Campyloxeninae, Hemiopinae, Lissominae, Oestodinae, Parablacinae, Physodactylinae, Pityobiinae, Subprotelaterinae, Tetralobinae. ZooKeys 2019, 839, 83-154. [CrossRef] [PubMed]

110. Bouchard, P.; Bousquet, Y.; Davies, A.E.; Alonso-Zarazaga, M.A.; Lawrence, J.F.; Lyal, C.H.C.; Newton, A.F.; Reid, C.A.M.; Schmitt, M.; Ślipiński, A.; et al. Family-group names in Coleoptera (Insecta). ZooKeys 2011, 88, 1-972.

111. Hyslop, J.A. Genotypes of the elaterid beetles of the world. Proc. U. S. Nat. Mus. 1921, 58, 621-673. [CrossRef]

112. Schenkling, S. Fam. Elateridae I. Coleopterorum Catalogus, Pars 80; W Junk: Berlin, Germany, 1925; pp. 1-263.

113. Cohen, K.M.; Finney, S.C.; Gibbard, P.L.; Fan, J.X. The ICS International Chronostratigraphic Chart. Episodes 2013, 36, 199-204. [CrossRef]

114. Leach, W.E. Entomology. In Edinburgh Encyclopaedia; Part I; Brewster, D., Blackwood, W., Waugh, J., Eds.; William Blackwood etc.: Edinburgh, UK, 1815; Volume 9, pp. 57-172.

115. Linnaeus, C. Systema Naturae per Regna Tria Naturae: Secundum Classes, Ordines, Genera, Species, Cum Characteribus, Differentiis, Synonymis, Locis. Tomus I, Editio Decima, Reformata; Laurentius Salvius: Stockholm, Sweden, 1758; pp. 1-823.

116. Candèze, E.C.A. Monographie des Élatérides. Tome premier. Mem. Soc. R. Sci. Liége 1857, 12, 1-400.

117. Eschscholtz, J.F. Elaterites, Eintheilung derselben in Gattungen. Entomol. Arch. 1829, 2, 31-35.

118. Latreille, P.A. Le règne Animal Distribué D'après son Organisation, Pour Servir de Base a L'histoire Naturelle des Animaux et D'introduction a L'anatomie Comparée. Par M le Baron Cuvier. Avec Figures Dessinées D'après Nature. Nouvelle Édition, Revue et Augmentée. Tome IV. Crustacés, Arachnides et Partie des Insectes; Déterville: Paris, France, 1829; pp. 1-584.

119. Germar, E.F. Coleopterorum Species Novae aut Minus Cognitae, Descriptionibus Illustratae; Volumen primum. Coleoptera. Cum Tab. Aen. II; Impensis JC Hendelii et Filii: Halae, Germany, 1823; pp. 1-624.

120. Korneev, V.A.; Cate, P. Names of insects described by Vladimir Gdalich Dolin. Vestn. Zool. 2005, 39, 9-26.

121. Sánchez-Ruiz, A. Catálogo bibliográfico de las especies de la familia Elateridae (Coleoptera) de la Península Ibérica e Islas Baleares. In Documentos Fauna Ibérica, 2nd ed.; Ramos, M.A., Ed.; Museo Nacional de Ciencias Naturales, CSIC: Madrid, Spain, 1996; pp. 1-265.

122. Hayek, C.M.F. A reclassification of the subfamily Agrypninae (Coleoptera: Elateridae). Bull. Brit. Mus. Nat. Hist. Entomol. 1973, 20, 1-309.

123. Laporte, F.L.N.C. Études entomologiques, ou descriptions d'insectes nouveaux et observations sur la synonymie. Rev. Entomol. 1838, 4, 5-60.

124. Fabricius, J.C. Supplementum Entomologiae Systematicae; Proft et Storch: Hafniae, Denmark, 1798; pp. 1-572.

125. Herbst, J.F.W. Kritisches Verzeichniß meiner Insektensammlung. Fortsetzung. Curculio Dytiscus. Arch. Insectengesch 1784, 5, 73-128.

126. Giebel, C.G.A. Deutschlands Petrefakten. Ein Systematisches Verzeichniss aller in Deutschland und den Angrenzenden Laendern Vorkommenden Petrefacten Nebst Angabe den Synonyme und Fundorte; Ambrosius Abel: Leipzig, Germany, 1852; pp. 1-706.

127. Handlirsch, A. Die Fossilen Insekten und Die Phylogenie der Rezenten Formen, Part V; Ein Handbuch fur Palaontologen und Zoologen; Wilhelm Engelmann: Leipzig, Germany, 1907; pp. 641-800.

128. Fleutiaux, E. Remarques et observations sur le catalogue des Elateridae, 1re partie, de M. S. Schenkling. Ann. Soc. Entomol. Fr. 1926, 95, 91-112.

129. ICZN (International Commission on Zoological Nomenclature). International Code of Zoological Nomenclature, 4th ed.; The International Trust for Zoological Nomenclature: London, UK, 1999; pp. 1-305.

130. Meunier, F. Die Insektenreste aus dem Lutetien von Messel bei Darmstadt. Abh. Hess. Geol. Landesanst. Darmstadt 1921, 7, 2-15.

131. Schimmel, R.; Tarnawski, D. Monograph of the subtribe Elaterina (Insecta: Coleoptera: Elateridae: Elaterinae). Genus 2010, 21, 325-487. 
132. Schimmel, R.; Tarnawski, D. Monograph of the monophylum Tetrigusina (Insecta: Coleoptera, Elateridae, Agrypninae). Polish Entomol. Monogr. 2012, 8, 1-288.

133. Keilbach, R. Bibliographie und Liste der Arten tierischer Einschlüße in fossilen Harzen sowie ihrer Aufbewahrungsorte. Dtsch. Entomol. Z. 1982, 29, 129-286. [CrossRef]

134. Berthold, A.A. Natürliche Familien des Thierreichs. Aus dem Französischen mit Anmerkungen und Zusätzen; Landes-Industrie-Comptoir: Weimar, Germany, 1827; pp. 1-606.

135. Heer, O. Le Monde Primitif de la Suisse; H. Georg: Genève, Switzerland; Basel, Switzerland, 1872; pp. 1-801.

136. Heer, O. The Primaeval World of Switzerland; Heywood, J., Ed.; Longmans, Green, and Co.: London, UK, 1876; Volume 2, pp. 1-324.

137. Heer, O. Die Urwelt der Schweiz, 2nd ed.; Friedrich Schulthess: Zürich, Switzerland, 1883; pp. 1-713.

138. Gistel, J.N.F.X. Faunula monacensis cantharologica. Isis Oken 1848, 7, 4-6.

139. Dejean, P.F.M.A. Catalogue des Coléoptères de la Collection de M. le Comte Dejean; Livraisons 1-2; Méquignon-Marvis: Paris, France, 1833; pp. 1-176.

140. Arnett, R.H. The systematic position of Melanactes and Pseudomelanactes (Coleoptera, Elateridae). Entomol. News 1967, 78, $110-111$.

141. Mathieu, J.M. Revision of the genus Melanactes, with a proposed new genus (Coleoptera, Elateridae). Am. Midl. Nat. 1961, 65, 459-480. [CrossRef]

142. Fairmaire, L. Descriptions de Coléoptères de l'Indo-Chine. Ann. Soc. Entomol. Fr. 1888, 6, 333-378.

143. Arnett, R.H. A review of the Nearctic Adelocerina (Coleoptera: Elateridae, Pyrophorinae, Pyrophorini). Wasmann J. Biol. 1952, 10, 103-126.

144. LeConte, J.L. Revision of the Elateridæ of the United States. Trans. Am. Phil. Soc. 1853, 10, 405-508.

145. Candèze, E.C.A. Monographie des Élatérides. Tome quatrième. Mem. Soc. R. Sci. Liége 1863, 17, 1-534.

146. Billberg, G.J. Enumeratio insectorum in Museo Gust. Joh. Billberg; Gadel: Stockholm, Sweden, 1820; pp. 1-138.

147. Haupt, H. Beitrag zur Kenntnis der eozänen Arthropodenfauna des Geiseltales. Nova Acta Leopold. 1956, 18, 1-89.

148. Candèze, E.C.A. Monographie des Élatérides. Tome second. Mem. Soc. R. Sci. Liége 1859, 14, 1-543.

149. Fabricius, J.C. Systema Eleutheratorium Secundum Ordines, Genera, Species Adiectis Synonymis, Locis, Observationibus, Descriptionibus; Tom II; Bibliopolii Academici Novi: Kiliae, Germany, 1801; pp. 1-687.

150. Scopoli, J.A. Entomologia Carniolica: Exhibens Insecta Carnioliae Indigena et Distributa in Ordines, Genera, Species, Varietates: Methodo Linnaeana; Johann Trattner: Vienna, Austria, 1763; pp. 1-420.

151. Wickham, H.F. Supplement to Catalogue of North American Coleoptera described as fossils. In Supplement 1919 to 1924 (Inclusive) to Catalogue of the Coleoptera of America, North of Mexico; Leng, C.W., Mutchler, A.J., Eds.; Sherman: Mt. Vernon, NY, USA, 1927; pp. $55-56$.

152. Candèze, E.C.A. Monographie des Élatérides. Tome troisième. Mem. Soc. R. Sci. Liége 1860, 15, 1-512.

153. Fabricius, J.C. Entomologia Systematica Emendata et Aucta. Secundum Classes, Ordines, Genera, Species Adjectis Synonimis, Locis, Observationibus, Descriptionibus; Christian Gottlob: Copenhagen, Denmark, 1792; pp. 1-330.

154. Birket-Smith, S.J.R. Fossil insects from Spitsbergen. Acta Arct. 1977, 19, 1-42.

155. Etzler, F.E. Generic reclassification of Limonius Eschscholtz, 1829 (Elateridae: Dendrometrinae) sensu Candèze 1860 of the world. Zootaxa 2019, 4683, 301-335. [CrossRef] [PubMed]

156. Scudder, S.H. Canadian fossil insects, myriapods and arachnids, 2. The Coleoptera hitherto found fossil in Canada. Geological Survey of Canada. Contrib. Can. Palaeontol. 1895, 2, 27-56.

157. Scudder, S.H. Adephagous and clavicorn Coleoptera from the Tertiary deposits at Florissant, Colorado with descriptions of a few other forms and a systematic list of the non-rhynchophorus Tertiary Coleoptera of North America. Monogr. U. S. Geol. Surv. 1900, $40,1-148$.

158. Heyden, C.V. Gliederthiere aus der Braunkohle des Niederrhein's, der Wetterau und der Rohn. Palaeontographica 1862, 10, 62-82.

159. Charpentier, T. Horae Entomologicae, Adjectis Tabulis Novem Coloratis; A. Gosohorsky: Wratislaviae, Poland, $1825 ;$ pp. 1-255.

160. Kundrata, R.; Musalkova, M.; Kubaczkova, M. Annotated catalogue of the click-beetle tribe Dimini (Coleoptera: Elateridae: Dendrometrinae). Zootaxa 2018, 4412, 1-75. [CrossRef] [PubMed]

161. Schwarz, O. Coleoptera. Fam. Elateridae. In Genera Insectorum; Fascicules 46A, 46B; Wytsman, P.A., Ed.; P. Wytsman: Brussels, Belgium, 1906; Volume VII, pp. 1-224.

162. Dillwyn, L.W. Memoranda Relating to Coleopterous Insects, Found in the Neighbourhood of Swansea; Murray, W.C., Rees, D., Eds.; Swansea, UK, 1829; pp. 1-75.

163. Stibick, J.N.L. A revision of the Hypnoidinae of the world (Col. Elateridae). Part I. Introduction, phylogeny, biogeography. The Hypnoidinae of North and South America. The genera Berninelsonius and Ligmargus. Eos 1976, 51, 143-223.

164. Stibick, J.N.L. A revision of the Hypnoidinae of the World (Col. Elateridae). Part V. The Hypnoidinae of New Zealand, fossil Hypnoidinae, postscript and indexes. Eos 1981, 55-56, 227-294.

165. Scudder, S.H. The Tertiary Insects of North America. Rep. U. S. Geol. Surv. Terr. 1890, 13, 1-734.

166. Hope, F.W. A monograph on the coleopterous family Phyllophoridae. Proc. Zool. Soc. Lond. 1842, 10, 73-79.

167. Latreille, P.A. Distribution méthodique et naturelle des genres de diverses tribus d'insectes coléopteres, de la famille des serricornes. Ann. Soc. Entomol. Fr. 1834, 3, 113-170.

168. Olivier, G.A. Entomologie, ou Histoire Naturelle des Insectes, Avec Leurs Caractères Génériques et Spécifiques, Leur Description, Leur Synonymie, et leur Figure Enluminée. Coléoptères; Tome Deuxième; Baudouin: Paris, France, 1790; pp. 1-458. 
169. Drury, D. Illustrations of Natural History. Wherein Are exhibited Upwards of Two Hundred and Forty Figures of Exotic Insects, according to Their Different Genera; Very Few of Which Have Hitherto Been Figured by Any Author, Being Engraved and Coloured from Nature, with the Greatest Accurancy, and under the Author's Own Inspection, on Fifty Copper Plates. With a Particular Description of each Insect: Interspersed with Remarks and Reflections on the Nature and Properties of Many of Them; Benjamin White: London, UK, 1773; Volume 2, pp. 1-90.

170. Gistel, J.N.F.X. Fauna der Vorwelt mit Steter Beruecksichtigung der Lebenden Thiere. Zweiter Band: Gliederthiere. Erste Abtheilung: Insecten und Spinnen; Brockhaus: Leipzig, Germany, 1856; pp. 1-512.

171. Heer, O. Recherches sur le Climat et la Végétation du Pays Tertiaire; Jean Wurster \& Comp.: Winterthur, Switzerland, 1861; pp. 1-220.

172. Scudder, S.H. The Fossil Insects of the Green River Shales. Bull. U. S. Geol. Geogr. Surv. Terr. 1878, 4, 747-776.

173. LeConte, J.L. Classification of the Coleoptera of North America. Prepared for the Smithsonian Institution, Part I. Smithson. Misc. Coll. 1861, 136, 1-214.

174. LeConte, J.L. New species of North American Coleoptera. Smithson. Misc. Coll. 1863, 167, 1-86.

175. Say, T. Descriptions of coleopterous insects collected in the late expedition to the Rocky Mountains, performed by order of Mr. Calhoun, Secretary of War, under the command of Major Long. J. Acad. Nat. Sci. Phila. 1823, 3, 139-282.

176. Scudder, S.H. The first discovered traces of fossil insects in the American Tertiaries. Bull. U. S. Geol. Geogr. Surv. Terr. 1877, 3 , 741-762.

177. Roache, L.C. A revision of the genus Oxygonus LeConte with a description of one new species (Coleoptera: Elateridae) (Tribe: Agriotini). Coleopt. Bull. 1963, 15, 101-108.

178. Schimmel, R.; Tarnawski, D.; Han, T.; Platia, G. Monograph of the new tribe Selatosomini from China (Elateridae: Denticollinae). Part I: Genera Pristilophus Latreille, 1834 stat. nov., Selatosomus Stephens, 1830, Warchalowskia (Tarnawski, 1995) stat. nov., and Sinophotistus gen. nov. Pol. Entomol. Monogr. 2015, 11, 1-328.

179. Stephens, J.F. Illustrations of British Entomology; or, a Synopsis of Indigenous Insects: Containing Their Generic and Specific Distinctions; with an Account of Their Metamorphoses, Times of Appearance, Localities, Food, and Economy, As Far As Practicable; Mandibulata; Baldwin and Cradock: London, UK, 1830; Volume 3, pp. 1-374.

180. Jakobson, G.G. Zhuki Rossii i Zapadnoy Evropy. Fasc. 10; Izdaniye A. Devriena: St. Petersburg, Russia, 1913 ; pp. $721-864$.

181. Fabricius, J.C. Systema Entomologiae, Sistens Insectorvm Classes, Ordines, Genera, Species, Adiectis Synonymis, Locis, Descriptionibus, Observationibvs; Officina Libraria Kortii: Flensbvrgi et Lipsiae, Germany, 1775; pp. 1-832.

182. Laporte, F.L.N.C. Histoire Naturelle des Insectes Coléoptères. Avec une Introduction Renfermant L'anatomie et la Physiologie des Animaux Articulés, par M. Brullé. Tome Premier. Histoire Naturelle des Animaux Articulés, Annelides, Crustacés, Arachnides, Myriapodes et Insectes; P. Duménil: Paris, France, 1840; pp. 1-324.

183. Germar, E.F. Bemerkungen über Elateriden. Z. Entomol. 1844, 5, 133-192.

184. Panzer, W.G.F. Faunae Insectorum Germanicae Initia Oder Deutschlands Insecten; Heft 6; Felsecker: Nürnberg, Germany, 1793; pp. $1-24$.

185. Wickham, H.F. Second supplement to Catalogue of North American Coleoptera described as fossils. In Second and Third Supplements 1925 to 1932 (Inclusive) to Catalogue of the Coleoptera of America, North of Mexico; Leng, C.W., Mutchler, A.J., Eds.; Sherman: Mt. Vernon, NY, USA, 1933; pp. 103-105.

186. Fletcher, T.B. Indian fossil insects. In Proceedings of the Third Entomological Meeting, Pusa, India, 3-15 February 1919; Agricultural Research Institute: Pusa, India, 1920; Volume 3, pp. 983-990.

187. Zherikhin, V.V. Various ways of examining past faunistic complexes. Trudy Paleontol. Inst. Akad. Nauk SSSR 1978, 165, 1-198.

188. Poinar, G. Life in Amber; Stanford University Press: Stanford, CA, USA, 1992; pp. 1-350.

189. Ross, A.J.; York, P.V. A list of type and figured specimens of insects and other inclusions in Burmese amber. Bull. Nat. Hist. Mus. Geol. Ser. 2000, 56, 11-20.

190. Peris, D.; Háva, J. New species from Late Cretaceous New Jersey amber and stasis in subfamily Attageninae (Insecta: Coleoptera: Dermestidae). J. Paleontol. 2016, 90, 491-498. [CrossRef]

191. Gurjeva, E.L. Novaya triba zhukov-shchelkunov Megapenthini tribus n. (Coleoptera, Elateridae). Zool. Zhurnal 1973, 52, 448-451.

192. Kiesenwetter, E.A.H. Naturgeschichte der Insecten Deutschland. Erste Abtheilung. Coleoptera. Vierter Band; Nicolaische Verlagsbuchhandlung: Berlin, Germany, 1858; pp. 179-386.

193. Fleutiaux, E. Révision des Élatérides (Coléoptères) de l’Indo-Chine Française. Première Partie. Notes Entomol. Chin. 1947, 11, 233-420.

194. Candèze, E.C.A. Élatérides nouveaux. Ann. Soc. Entomol. Belg. 1878, 21, 135-143.

195. Redtenbacher, G. Quaedam Genera et Species Coleopterorum Archiducatus Austriae Nondum Descriptorum; Typis Caroli Ueberreuter: Vindobonae, Austria, 1842; pp. 1-31.

196. Germar, E.F. Bemerkungen über Elateriden. Z. Entomol. 1840, 2, 241-278.

197. Laporte, F.L.N.C. Études entomologiques, ou descriptions d'insectes nouveaux et observations sur la synonymie. Rev. Entomol. $1835,3,157-181$.

198. Dalman, J.W. Insectorum Nova Genera. Ephemerides Entomologicae; P.A. Norstedt: Holmiae, Sweden, 1824; pp. 1-36.

199. Legalov, A.A. Fossil Mesozoic and Cenozoic weevils (Coleoptera, Obrienioidea, Curculionoidea). Paleontol. J. 2015, 49 , 1442-1513. [CrossRef]

200. Legalov, A.A. Fossil History of Curculionoidea (Coleoptera) from the Paleogene. Geosciences 2020, 10, 358. [CrossRef] 
201. Schwarz, O. Ueber die Elateriden-Gattungen Protelater Sharp und Anaspasis Cand. und ihre systematische Stellung nebst Beschreibung einer neuen Gattung und Art. Dtsch. Entomol. Z. 1902, 1902, 364-366.

202. Sharp, D. On the Elateridae of New Zealand. Ann. Mag. Nat. Hist. 1877, 19, 396-487. [CrossRef]

203. Nakane, T.; Kishii, T. On the subfamilies of Elateridae from Japan (Coleoptera). Kontŷि 1956, 24, $201-206$.

204. Thomson, C.G. Skandinaviens Coleoptera, Synoptiskt Bearbetade, Tomus I; Tryckt uti Berlingska Boktryckeriet: Lund, Sweden, 1859; pp. $1-290$.

205. Stibick, J.N.L. The generic classification of the Negastriinae (Coleoptera: Elateridae). Pac. Insects 1971, 13, 371-390.

206. Say, T. Description of new North American insects and observations on some already described. Trans. Am. Phil. Soc. 1839, 6, 155-190. [CrossRef]

207. Lacordaire, J.T. Histoire Naturelle des Insectes. Genera des Coléoptères ou Exposé Méthodique et Critique de Tous les Genres Proposés Jusqu'ici dans cet Ordre D'insectes. Tome Quatrième Contenant les Familles des Buprestides, Throscides, Eucnémides, Élatérides, Cébrionides, Cérophytides, Rhipicérides, Dascyllides, Malacodermes, Clérides, Lyméxylones, Cupésides, Ptiniores, Bostrichides et Cissides; Librairie Encyclopédique de Roret: Paris, France, 1857; pp. 1-579.

208. Geoffroy, E.L. Histoire Abregée des Insectes qui se Trouvent aux Environs de Paris, dans Laquelle ces Animaux Sont Ranges Suivant un ordre Méthodique. Tome Premier; Durand: Paris, France, 1762; pp. 1-523.

209. Hyslop, J.A. The phylogeny of the Elateridae based on larval characters. Ann. Entomol. Soc. Am. 1917, 10, 241-263. [CrossRef]

210. Stibick, J.N.L. Classification of the Elateridae (Coleoptera). Relationships and classification of the subfamilies and tribes. Pac. Insects 1979, 20, 145-186.

211. Huber, C.; Marggi, W.; Menkveld-Gfeller, U. Dolinelater nom. nov., a substitute name for Idiomorphus Dolin, 1980 (Coleoptera, Elateridae). Contrib. Nat. Hist. 2017, 34,1-4.

212. Handlirsch, A. Neue Untersuchungen über die fossilen Insekten mit Ergänzungen und Nachträgen sowie Ausblicken auf phylogenetische, palaeogeographische und allgemein biologische Probleme. II. Teil. Ann. Naturhist. Mus. Wien. 1938, 49, 1-240.

213. Phillips, J. Geology of Oxford and the Valley of the Thames; Clarendon Press: Oxford, UK, 1871; pp. 1-523.

214. Dong, F.B.; Cai, C.Y.; Huang, D.Y. Revision of five Mesozoic beetles from southern China. Acta Pal. Sin. $2011,50,481-491$.

215. Jell, P.A. The fossil insects of Australia. Mem. Qld. Mus. 2004, 50, 1-124.

216. Cockerell, T.D.A. The fauna of the Sunchal (or Margas Verdes) Formation, Northern Argentina. Am. Mus. Novit. 1936, 886, 1-9.

217. Coram, R.A.; Jepson, J.E. Fossil Insects of the Purbeck Limestone Group of Southern England: Palaeoentomology from the Dawn of the Cretaceous; Siri Scientific Press: Manchester, UK, 2012; pp. 1-144.

218. Yu, Y.; Ślipiński, A.; Lawrence, J.F.; Yan, E.; Ren, D.; Pang, H. Reconciling past and present: Mesozoic fossil record and a new phylogeny of the family Cerophytidae (Coleoptera: Elateroidea). Cret. Res. 2019, 99, 51-70. [CrossRef]

219. Yan, E.V.; Zhang, H. New beetle species of the formal genus Artematopodites (Coleoptera: Polyphaga), with remarks on the taxonomic position of the genera Ovivagina and Sinonitidulina. Paleontol. J. 2010, 44, 451-456. [CrossRef]

220. Heyden, C.V. Fossile Insekten aus der Rheinischen Braunkhohle. Palaeontographica 1859, 8, 1-15.

221. Crowson, R.A. The evolutionary history of Coleoptera, as documented by fossil and comparative evidence. Atti Congr. Naz. Ital. Ent. 1975, 10, 47-90.

222. Hörnschemeyer, T. New species of Electribius Crowson 1973 (Coleoptera: Artematopodidae) from Baltic amber. Paläontol. Z. 1998, 72, 299-306. [CrossRef]

223. Štys, P. Revision of fossil and pseudofossil Enicocephalidae (Heteroptera). Acta Entomol. Bohemoslov. 1969, 66, 352-365.

224. Bellamy, C.L. Buprestidae (Coleoptera) from amber deposits: A brief review and family switch. Coleopt. Bull. $1995,49,175-177$.

225. Ross, A.J. Amber, the Natural Time Capsule; The Natural History Museum: London, UK, 1998; pp. 1-73.

226. Bellamy, C.L. World Catalogue and Bibliography of the Jewel Beetles (Coleoptera: Buprestoidea); Volume 1: Introduction; Fossil Taxa; Schizopodidae; Buprestidae: Julodinae-Chrysochroinae: Poecilonotini; Pensoft Publishers: Sofia, Bulgaria, 2008 ; pp. 1-625.

227. Ding, Q.; Labandeira, C.C.; Ren, D. Biology of a leaf miner (Coleoptera) on Liaoningocladus boii (Coniferales) from the Early Cretaceous of northeastern China and the leaf-mining biology of possible insect culprit clades. Arthropod Syst. Phylogeny 2014, 72, 281-308.

228. Calder, A.A. Click Beetles: Genera of the Australian Elateridae (Coleoptera). Monographs on Invertebrate Taxonomy; CSIRO Publishing: Victoria, Australia, 1996; Volume 2, pp. 1-401.

229. Grimaldi, D.; Engel, M. Evolution of the Insects; Cambridge University Press: New York, NY, USA; Cambridge, UK, 2005 ; pp. 1-755.

230. McKenna, D.D.; Wild, A.L.; Kanda, K.; Bellamy, C.L.; Beutel, R.G.; Caterino, M.C.; Farnum, C.W.; Hawks, D.C.; Ivie, M.A.; Jameson, M.L.; et al. The beetle tree of life reveals that Coleoptera survived end-Permian mass extinction to diversify during the Cretaceous terrestrial revolution. Syst. Entomol. 2015, 40, 835-880. [CrossRef]

231. Bocak, L.; Kundrata, R.; Andújar Fernández, C.; Vogler, A.P. The discovery of Iberobaeniidae (Coleoptera: Elateroidea): A new family of beetles from Spain, with immatures detected by environmental DNA sequencing. Proc. R. Soc. B 2016, 283, 20152350. [CrossRef] [PubMed]

232. Hunt, T.; Bergsten, J.; Levkanicova, Z.; Papadopoulou, A.; St John, O.; Wild, R.; Hammond, P.M.; Ahrens, D.; Balke, M.; Caterino, M.S.; et al. A comprehensive phylogeny of beetles reveals the evolutionary origins of a superradiation. Science 2007, 318, 1913-1916. [CrossRef] [PubMed]

233. Chang, H.L.; Muona, J.; Pu, H.; Xu, L.; Wang, C.; Teräväinen, M.; Ren, D.; Yang, Q.; Zhang, X.; Jia, S. Chinese Cretaceous larva exposes a southern Californian living fossil (Insecta, Coleoptera, Eucnemidae). Cladistics 2016, 32, $211-214$. 
234. Li, Y.D.; Tihelka, E.; Liu, Z.H.; Huang, D.Y.; Cai, C.Y. Muonabuntor gen. nov., a new genus of false click beetles from midCretaceous Burmese amber (Coleoptera: Elateroidea: Eucnemidae). Palaeoentomology 2020, 3, 399-406. [CrossRef]

235. Zhang, S.-Q.; Che, L.-H.; Li, Y.; Liang, D.; Pang, H.; Ślipiński, A.; Zhang, P. Evolutionary history of Coleoptera revealed by extensive sampling of genes and species. Nat. Commun. 2018, 9, 1-11. [CrossRef] [PubMed]

236. Muona, J. Throscidae (Coleoptera) relationships, with descriptions of new fossil genera and species. Zootaxa 2019, 4576, 521-543. [CrossRef]

237. Li, Y.D.; Zhao, Y.-C.; Huang, D.Y.; Cai, C.Y. A new throscid from mid-Cretaceous Burmese amber (Coleoptera: Elateroidea: Throscidae). Hist. Biol. 2020, in press. [CrossRef]

238. Muona, J. The late Cretaceous amber Eucnemidae fossils from Myanmar. Ent. Bl. Col. 2020, 116, 1-27.

239. Li, Y.D.; Huang, D.Y.; Cai, C.Y. New genera and species of the family Throscidae (Coleoptera: Elateroidea) in Mid-Cretaceous Burmese amber. Insects 2021, 12, 63. [CrossRef] [PubMed]

240. Li, H.; Chang, H.L.; Muona, J.; Zhao, Y.; Ren, D. Subfamily Anischiinae (Coleoptera: Eucnemidae) in early Cretaceous of Northeast China. Insects 2021, 12, 105. [CrossRef] [PubMed]

241. Alekseev, V.I.; Ellenberger, S. A new beetle family, Mysteriomorphidae fam. nov. (Coleoptera: Elateriformia incertae sedis), from mid-Cretaceous amber of northern Myanmar. Palaeoentomology 2019, 2, 482-490. [CrossRef]

242. Peris, D.; Kundrata, R.; Delclòs, X.; Mähler, B.; Ivie, M.A.; Rust, J.; Labandeira, C.C. Unlocking the mystery of the mid-Cretaceous Mysteriomorphidae (Coleoptera: Elateroidea) and modalities in transiting from gymnosperms to angiosperms. Sci. Rep. 2020, 10, 16854. [CrossRef]

243. Lawrence, J.F.; Ślipiński, A.; Seago, A.E.; Thayer, M.K.; Newton, A.F.; Marvaldi, A.E. Phylogeny of the Coleoptera based on morphological characters of adults and larvae. Ann. Zool. 2011, 61, 1-217. [CrossRef]

244. Gurjeva, E.L. Stroenie grudnogo otdela zhukov-shchelkunov (Coleoptera, Elateridae) i znachenie ego priznakov dlya sistemy semeistva [Thoracic structure of click beetles (Coleoptera, Elateridae) and the significance of the structural characters for the system of the family]. Entomol. Obozr. 1974, 53, 96-113.

245. Meyer, H.W. The Fossils of Florissant; Smithsonian Books: Washington, DC, USA, 2003; pp. 1-258.

246. Fikáček, M.; Schmied, H. Insect fauna of the Late Miocene locality of Öhningen (Germany) less diverse than reported: An example of the hydrophilid beetles (Coleoptera). J. Paleontol. 2013, 87, 427-443. [CrossRef]

247. Tarasov, S.; Vaz-de-Mello, F.Z.; Krell, F.; Dimitrov, D. A review and phylogeny of Scarabaeine dung beetle fossils (Coleoptera: Scarabaeidae: Scarabaeinae), with the description of two Canthochilum species from Dominican amber. PeerJ 2016, 4, e1988. [CrossRef] [PubMed]

248. Ponomarenko, A.G. The geological history of beetles. In Biology, Phylogeny and Classification of Coleoptera: Papers Celebrating the 80th Birthday of Roy A. Crowson; Pakaluk, J., Ślipiński, A., Eds.; Muzeum i Instytut Zoologii PAN: Warszawa, Poland, 1995; pp. 87-155.

249. Poinar, G. Associations between fossil beetles and other organisms. Geosciences 2019, 9, 184. [CrossRef]

250. Peris, D.; Ruzzier, E.; Perrichot, V.; Delclòs, X. Evolutionary and paleobiological implications of Coleoptera (Insecta) from Tethyan-influenced Cretaceous ambers. Geosci. Front. 2016, 7, 695-706. [CrossRef]

251. Peris, D. Coleoptera in amber from Cretaceous resiniferous forests. Cret. Res. 2020, 113, 104484. [CrossRef]

252. Peris, D.; Rust, J. Cretaceous beetles (Insecta: Coleoptera) in amber: The palaeoecology of this most diverse group of insects. Zool. J. Linn. Soc. 2020, 189, 1085-1104. [CrossRef]

253. Kirejtshuk, A.G.; Azar, D. New taxa of beetles (Insecta, Coleoptera) from Lebanese amber with evolutionary and systematic comments. Alavesia 2008, 2, 15-46.

254. Boucher, S.; Bai, M.; Wang, B.; Zhang, W.; Yang, X. +Passalopalpidae, a new family from the Cretaceous Burmese amber, as the possible sister group of Passalidae Leach (Coleoptera: Scarabaeoidea). Cret. Res. 2016, 64, 67-78. [CrossRef]

255. Bao, T.; Walczyńska, K.S.; Moody, S.; Wang, B.; Rust, J. New family Apotomouridae fam. nov. (Coleoptera: Tenebrionoidea) from lower Cenomanian amber of Myanmar. Cret. Res. 2018, 91, 14-19. [CrossRef]

256. Clarke, D.J.; Limaye, A.; McKenna, D.D.; Oberprieler, R.G. The weevil fauna preserved in Burmese amber-Snapshot of a unique, extinct lineage (Coleoptera: Curculionoidea). Diversity 2019, 11, 1. [CrossRef]

257. Li, Y.D.; Kundrata, R.; Tihelka, E.; Liu, Z.H.; Huang, D.Y.; Cai, C.Y. Cretophengodidae, a new Cretaceous beetle family, sheds light on the evolution of bioluminescence. Proc. R. Soc. B: Biol. Sci. 2021, 288, 20202730. [CrossRef]

258. Arriaga-Varela, E.; Brunke, A.; Girón, J.C.; Szawaryn, K.; Bruthansová, J.; Fikáček, M. Micro-CT reveals hidden morphology and clarifies the phylogenetic position of Baltic amber water scavenger beetles (Coleoptera: Hydrophilidae). Hist. Biol. 2019, in press. [CrossRef]

259. Rasnitsyn, A.P.; Ross, A.J. A prelimanary list of arthropod families present in the Burmese amber collection at The Natural History Museum, London. Bull. Nat. Hist. Mus. Geol. Ser. 2000, 56, 21-24. 